\begin{tabular}{|c|l|}
\hline Title & IWASA WA THEORY OF DE RHAM (phi, Gamma)-MODULES OV ER THE ROBBA RING \\
\hline Author(s) & Nakamura, Kentaro \\
\hline Citation & $\begin{array}{l}\text { Journal of the institute of mathematics of jussieu, 13(1), 65-118 } \\
\text { https://doi.org/10.1017/S1474748013000078 }\end{array}$ \\
\hline Issue Date & 2014_01 \\
\hline Doc URL & http://hdl.handle.net/2115/57802 \\
\hline Type & article \\
\hline File Information & JIMJ_13_65-.pdf \\
\hline
\end{tabular}

Instructions for use 


\section{Journal of the Institute of Mathematics of Jussieu}

http://journals.cambridge.org/JMJ

Additional services for Journal of the Institute of

Mathematics of Jussieu:

Email alerts: $\underline{\text { Click here }}$

Subscriptions: $\underline{\text { Click here }}$

Commercial reprints: $\underline{\text { Click here }}$

Terms of use : $\underline{\text { Click here }}$

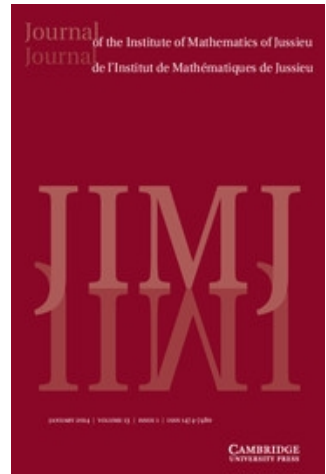

\section{Iwasawa theory of de Rham I\$(Ivarphi , IGamma )|\$- modules over the Robba ring}

Kentaro Nakamura

Journal of the Institute of Mathematics of Jussieu / Volume 13 / Issue 01 / January 2014, pp 65 - 118

DOI: 10.1017/S1474748013000078, Published online: 27 February 2013

Link to this article: http://journals.cambridge.org/abstract_S1474748013000078

How to cite this article:

Kentaro Nakamura (2014). Iwasawa theory of de Rham -modules over the Robba ring. Journal of the Institute of Mathematics of Jussieu, 13, pp 65-118 doi:10.1017/S1474748013000078

Request Permissions : $\underline{\text { Click here }}$ 


\title{
IWASAWA THEORY OF DE RHAM $(\varphi, \Gamma)$-MODULES OVER THE ROBBA RING
}

\author{
KENTARO NAKAMURA \\ Department of Mathematics, Hokkaido University, Sapporo 060-0810, Japan \\ (kentaro@math.sci.hokudai.ac.jp) \\ (Received 4 April 2012; revised 6 February 2013; accepted 6 February 2013; \\ first published online 27 February 2013)
}

\begin{abstract}
The aim of this article is to study the Bloch-Kato exponential map and the Perrin-Riou big exponential map purely in terms of $(\varphi, \Gamma)$-modules over the Robba ring. We first generalize the definition of the Bloch-Kato exponential map for all the $(\varphi, \Gamma)$-modules without using Fontaine's rings $\mathbf{B}_{\mathrm{crys}}, \mathbf{B}_{\mathrm{dR}}$ of $p$-adic periods, and then generalize the construction of the Perrin-Riou big exponential map for all the de Rham $(\varphi, \Gamma)$-modules and prove that this map interpolates our Bloch-Kato exponential map and the dual exponential map. Finally, we prove a theorem concerning the determinant of our big exponential map, which is a generalization of theorem $\delta(V)$ of Perrin-Riou. The key ingredients for our study are Pottharst's theory of the analytic Iwasawa cohomology and Berger's construction of $p$-adic differential equations associated to de $\operatorname{Rham}(\varphi, \Gamma)$-modules.
\end{abstract}

Keywords: $p$-adic Hodge theory; $(\varphi, \Gamma)$-module; $B$-pair

2010 Mathematics subject classification: Primary $11 \mathrm{~F} 80$

Secondary 11F $85 ; 11 \mathrm{~S} 25$

\section{Introduction}

\subsection{Introduction}

Let $p$ be a prime number, $K$ a finite extension of $\mathbb{Q}_{p}$, and $G_{K}$ the absolute Galois group of $K$. Let $\mathbf{B}_{\text {crys }}, \mathbf{B}_{e}:=\mathbf{B}_{\text {crys }}^{\varphi=1}, \mathbf{B}_{\mathrm{dR}}^{+}$, and $\mathbf{B}_{\mathrm{dR}}$ be Fontaine's rings of $p$-adic periods [14].

By the results of Fontaine [13], Cherbonnier and Colmez [8] and Kedlaya [22], the category of $p$-adic representations of $G_{K}$ is naturally embedded in the category of $\left(\varphi, \Gamma_{K}\right)$-modules over the Robba ring $\mathbf{B}_{\text {rig, } K}^{\dagger}$. The $(\varphi, \Gamma)$-modules corresponding to $p$-adic representations are called étale $(\varphi, \Gamma)$-modules.

The aim of this article is to study the Bloch-Kato exponential map and the Perrin-Riou big exponential map in the framework of $(\varphi, \Gamma)$-modules. In particular, we generalize the Perrin-Riou big exponential map to all the de Rham $(\varphi, \Gamma)$-modules.

\subsection{The Bloch-Kato exponential map}

For a $p$-adic representation $V$ of $G_{K}$, Bloch and Kato [7] defined a $\mathbb{Q}_{p}$-linear map

$$
\exp _{K, V}:=\delta_{1, V}: \mathbf{D}_{\mathrm{dR}}^{K}(V) \rightarrow \mathrm{H}^{1}(K, V),
$$


where we put $\mathbf{D}_{\mathrm{dR}}^{K}(V):=\left(\mathbf{B}_{\mathrm{dR}} \otimes_{\mathbb{Q}_{p}} V\right)^{G_{K}}$, as the first connecting homomorphism of the long exact sequence

$$
\begin{aligned}
& 0 \rightarrow \mathrm{H}^{0}(K, V) \rightarrow \mathrm{H}^{0}\left(K, \mathbf{B}_{e} \otimes_{\mathbb{Q}_{p}} V\right) \oplus \mathrm{H}^{0}\left(K, \mathbf{B}_{\mathrm{dR}}^{+} \otimes_{\mathbb{Q}_{p}} V\right) \rightarrow \mathrm{H}^{0}\left(K, \mathbf{B}_{\mathrm{dR}} \otimes_{\mathbb{Q}_{p}} V\right) \\
& \stackrel{\delta_{1, V}}{\longrightarrow} \mathrm{H}^{1}(K, V) \rightarrow \mathrm{H}^{1}\left(K, \mathbf{B}_{e} \otimes_{\mathbb{Q}_{p}} V\right) \oplus \mathrm{H}^{1}\left(K, \mathbf{B}_{\mathrm{dR}}^{+} \otimes_{\mathbb{Q}_{p}} V\right) \rightarrow \mathrm{H}^{1}\left(K, \mathbf{B}_{\mathrm{dR}} \otimes_{\mathbb{Q}_{p}} V\right) \\
& \stackrel{\delta_{2, V}}{\longrightarrow} \mathrm{H}^{2}(K, V) \rightarrow \mathrm{H}^{2}\left(K, \mathbf{B}_{e} \otimes_{\mathbb{Q}_{p}} V\right) \rightarrow 0
\end{aligned}
$$

associated to the short exact sequence obtained by tensoring $V$ with the so-called Bloch-Kato fundamental exact sequence

$$
0 \rightarrow \mathbb{Q}_{p} \stackrel{x \mapsto(x, x)}{\longrightarrow} \mathbf{B}_{e} \oplus \mathbf{B}_{\mathrm{dR}}^{+} \stackrel{(x, y) \mapsto x-y}{\longrightarrow} \mathbf{B}_{\mathrm{dR}} \rightarrow 0 .
$$

When $V$ is a de Rham representation, Kato [18] defined the dual exponential map

$$
\exp _{K, V^{\vee}(1)}^{*}: \mathrm{H}^{1}(K, V) \rightarrow \mathbf{D}_{\mathrm{dR}}^{K}(V)
$$

using Tate's pairing $\cup: \mathrm{H}^{1}(K, V) \times \mathrm{H}^{1}\left(K, V^{\vee}(1)\right) \rightarrow \mathbb{Q}_{p}$ and the canonical pairing $\mathbf{D}_{\mathrm{dR}}^{K}(V) \times \mathbf{D}_{\mathrm{dR}}^{K}\left(V^{\vee}(1)\right) \rightarrow K$. These maps describe the mysterious relationship between Galois objects and differential objects. In fact, when $V=\mathbb{Q}_{p}(1)$ or $V$ is the $p$-adic Tate module of an elliptic curve over $\mathbb{Q}$, Kato $[18,20]$ proved that the values of $\exp _{\mathbb{Q}_{p}\left(\zeta_{p^{n}}\right), V^{\vee}(1-k)}^{*}$ for suitable $k \leqq 0$ at some special arithmetic elements (i.e., cyclotomic units or Kato's elements obtained from his Euler system) can be described by using the special values of the $L$-functions associated to cyclotomic twists of $V$.

In this article, we first generalize the above long exact sequence and the definition of the Bloch-Kato exponential and the dual exponential maps for $\left(\varphi, \Gamma_{K}\right)$-modules over $\mathbf{B}_{\text {rig, } K}^{\dagger}$.

Fix a set $\left\{\zeta_{p^{n}}\right\}_{n \geqq 1} \subseteq \bar{K}$ such that $\zeta_{p} \neq 1, \zeta_{p}^{p}=1$ and $\zeta_{p^{n+1}}^{p}=\zeta_{p^{n}}$ for each $n \geqq 1$. Set $K_{n}:=K\left(\zeta_{p^{n}}\right), K_{\infty}:=\cup_{n} K_{n}$ and $\Gamma_{K}:=\operatorname{Gal}\left(K_{\infty} / K\right)$. Let $t:=\log (1+T) \in \mathbf{B}_{\text {rig, } K}^{\dagger}$ be the period of $\mathbb{Q}_{p}(1)$ determined by $\left\{\zeta_{p^{n}}\right\}_{n \geqq 1}$ (see $\S 2.1$ for the precise definition).

Let $D$ be a $\left(\varphi, \Gamma_{K}\right)$-module over $\mathbf{B}_{\text {rig, } K}^{\dagger}$. Taking the 'stalk at $\zeta_{p^{n}}-1$ ' $(n \geqq 1)$, we can define $K_{\infty}[[t]]:=\cup_{n} K_{n}[[t]]$-modules $\mathbf{D}_{\text {dif }}^{+}(D)$ and $\mathbf{D}_{\text {dif }}(D):=\mathbf{D}_{\text {dif }}^{+}(D)[1 / t]$ with semi-linear $\Gamma_{K}:=\operatorname{Gal}\left(K_{\infty} / K\right)$-action. Using the $\varphi, \Gamma_{K}$-actions, we can define cohomologies

$$
\mathrm{H}^{q}(K, D), \quad \mathrm{H}^{q}(K, D[1 / t]), \quad \mathrm{H}^{q}\left(K, \mathbf{D}_{\text {dif }}^{+}(D)\right) \quad \text { and } \quad \mathrm{H}^{q}\left(K, \mathbf{D}_{\text {dif }}(D)\right)
$$

which correspond to

$$
\mathrm{H}^{q}(K, V), \quad \mathrm{H}^{q}\left(K, \mathbf{B}_{e} \otimes_{\mathbb{Q}_{p}} V\right), \quad \mathrm{H}^{q}\left(K, \mathbf{B}_{\mathrm{dR}}^{+} \otimes_{\mathbb{Q}_{p}} V\right) \quad \text { and } \quad \mathrm{H}^{q}\left(K, \mathbf{B}_{\mathrm{dR}} \otimes_{\mathbb{Q}_{p}} V\right),
$$

respectively.

Our first result is the following theorem (more precisely, this is a combination of Theorems 2.8 and 2.21), which is the $(\varphi, \Gamma)$-module version of the above long exact sequence and its comparison with that of the étale case. 
Theorem 1.1. (1) We have the following functorial exact sequence:

$$
\begin{aligned}
& 0 \rightarrow \mathrm{H}^{0}(K, D) \rightarrow \mathrm{H}^{0}(K, D[1 / t]) \oplus \mathrm{H}^{0}\left(K, \mathbf{D}_{\text {dif }}^{+}(D)\right) \rightarrow \mathrm{H}^{0}\left(K, \mathbf{D}_{\text {dif }}(D)\right) \\
& \stackrel{\delta_{1, D}}{\longrightarrow} \mathrm{H}^{1}(K, D) \rightarrow \mathrm{H}^{1}(K, D[1 / t]) \oplus \mathrm{H}^{1}\left(K, \mathbf{D}_{\mathrm{dif}}^{+}(D)\right) \rightarrow \mathrm{H}^{1}\left(K, \mathbf{D}_{\mathrm{dif}}(D)\right) \\
& \stackrel{\delta_{2, D}}{\longrightarrow} \mathrm{H}^{2}(K, D) \rightarrow \mathrm{H}^{2}(K, D[1 / t]) \rightarrow 0
\end{aligned}
$$

(2) Let $D(V)$ be the $\left(\varphi, \Gamma_{K}\right)$-module over $\mathbf{B}_{\text {rig, }, K}^{\dagger}$ associated to $V$. Then, we have the following functorial isomorphisms.

(i) $\mathrm{H}^{q}(K, V) \stackrel{\sim}{\rightarrow} \mathrm{H}^{q}(K, D(V))$,

(ii) $\mathrm{H}^{q}\left(K, \mathbf{B}_{e} \otimes_{\mathbb{Q}_{p}} V\right) \stackrel{\sim}{\rightarrow} \mathrm{H}^{q}(K, D(V)[1 / t])$,

(iii) $\mathrm{H}^{q}\left(K, \mathbf{B}_{\mathrm{dR}}^{(+)} \otimes_{\mathbb{Q}_{p}} V\right) \stackrel{\sim}{\rightarrow} \mathrm{H}^{q}\left(K, \mathbf{D}_{\mathrm{dif}}^{(+)}(D(V))\right)$

for each $q \geqq 0$, and these comparison isomorphisms induce an isomorphism from the long exact sequence associated to $V$ to that associated to $D(V)$.

Remark 1.2. The isomorphism of (i) is due to Liu [23], and that of (iii) is due to Fontaine [15].

Remark 1.3. We construct this long exact sequence purely in terms of $(\varphi, \Gamma)$-modules without using Fontaine's rings $\mathbf{B}_{\mathrm{crys}}, \mathbf{B}_{\mathrm{dR}}^{+}$and $\mathbf{B}_{\mathrm{dR}}$. As will be shown in this article, this fact enables us to reprove some results concerning Bloch-Kato or Perrin-Riou exponential maps more directly.

Remark 1.4. In fact, in $\S 2.5$, we prove the above comparison result (2) in a more general setting. In [5], Berger defined a notion of $B$-pairs using $\mathbf{B}_{e}, \mathbf{B}_{\mathrm{dR}}^{+}$and $\mathbf{B}_{\mathrm{dR}}$, whose category naturally contains the category of $p$-adic representations of $G_{K}$, and established an equivalence of categories between the category of $B$-pairs and that of $\left(\varphi, \Gamma_{K}\right)$-modules over $\mathbf{B}_{\mathrm{rig}, K}^{\dagger}$. In $\S 2.5$, we prove the comparison isomorphisms for all the $B$-pairs (see Theorem 2.21).

As in the case of $p$-adic representations, we define the Bloch-Kato exponential map of $D$ as the connecting homomorphism of the above exact sequence

$$
\exp _{K, D}:=\delta_{1, D}: \mathbf{D}_{\mathrm{dR}}^{K}(D) \rightarrow \mathrm{H}^{1}(K, D),
$$

where we put $\mathbf{D}_{\mathrm{dR}}^{K}(D):=\mathrm{H}^{0}\left(K, \mathbf{D}_{\text {dif }}(D)\right)$. When $D$ is a de Rham $(\varphi, \Gamma)$-module, then we also define the dual exponential map

$$
\exp _{K, D^{\vee}(1)}^{*} \mathrm{H}^{1}(K, D) \rightarrow \mathbf{D}_{\mathrm{dR}}^{K}(D)
$$

in the same way as in the case of $p$-adic representations.

\subsection{The Perrin-Riou big exponential map}

To construct a $p$-adic $L$-function for a $p$-adic Galois representation $V$ coming from a motive, it is crucial to $p$-adically interpolate the special values of the complex $L$-functions associated to cyclotomic twists of $V$. Since the Bloch-Kato exponential map and the dual exponential map relate some arithmetic elements in Galois cohomology 
groups with the special values of the $L$-functions, it is crucial to $p$-adically interpolate the Bloch-Kato exponential map and the dual exponential map for the construction of the $p$-adic $L$-function and for relating the $p$-adic $L$-function with the Selmer group.

Let $\Lambda:=\mathbb{Z}_{p}\left[\left[\Gamma_{K}\right]\right]$ be the Iwasawa algebra of $\Gamma_{K}$, and let $\Lambda_{\infty}$ be the $\mathbb{Q}_{p}$-valued distribution algebra of $\Gamma_{K}$ (see $\S 3.1$ for the precise definition). For a $p$-adic representation $V$ of $G_{K}$, Perrin-Riou [28] defined a $\Lambda$-module

$$
\mathbf{H}_{\mathrm{IW}}^{q}(K, V):=\left(\lim _{n} \mathrm{H}^{q}\left(K_{n}, T\right)\right) \otimes_{\mathbb{Z}_{p}} \mathbb{Q}_{p},
$$

called the Iwasawa cohomology of $V$, where $T$ is a $G_{K}$-stable $\mathbb{Z}_{p}$-lattice of $V$ and the transition map is the corestriction map. This $\Lambda$-module $p$-adically interpolates $\mathrm{H}^{q}(L, V(k))$ for any $L=K, K_{n}$ and $k \in \mathbb{Z}$, i.e., we have a natural projection map

$$
\operatorname{pr}_{L, V(k)}: \mathbf{H}_{\mathrm{Iw}}^{q}(K, V) \rightarrow \mathrm{H}^{q}(L, V(k))
$$

for each $L$ and $k$. When $K$ is unramified over $\mathbb{Q}_{p}$ and $V$ is a crystalline representation of $G_{K}$, Perrin-Riou [29] constructed a system of functorial $\Lambda_{\infty}$-morphisms

$$
\Omega_{V, h}:\left(\Lambda_{\infty} \otimes_{\mathbb{Q}_{p}} \mathbf{D}_{\text {crys }}^{K}(V)\right)^{\widetilde{\Delta}=0} \rightarrow \Lambda_{\infty} \otimes_{\Lambda}\left(\mathbf{H}_{\mathrm{Iw}}^{1}(K, V) / \mathbf{H}_{\mathrm{IW}}^{1}(K, V)_{\Lambda \text {-torsion }}\right)
$$

for each $h \geqq 1$ such that $\mathrm{Fil}^{-h} \mathbf{D}_{\mathrm{dR}}^{K}(V)=\mathbf{D}_{\mathrm{dR}}^{K}(V)$, and proved that this interpolates $\exp _{L, V(k)}$ and $\exp _{L, V^{\vee}(1+k)}^{*}$ for any $L=K_{n}, K$ and for suitable $k$. Here, the source of the map $\Omega_{V, h}$ is a $\Lambda_{\infty}$-module which $p$-adically interpolates $\mathbf{D}_{\mathrm{dR}}^{L}(V(k))$ for any $L$ and $k$. This map $\Omega_{V, h}$ is the most important ingredient for her study of $p$-adic $L$-functions [30].

The main purpose of this article is to generalize the map $\Omega_{V, h}$ to all the de Rham $(\varphi, \Gamma)$-modules. For this generalization, the following two notions are essential:

(1) Pottharst's theory of the analytic Iwasawa cohomology,

(2) Berger's construction of $p$-adic differential equations associated to de Rham $(\varphi, \Gamma)$-modules.

As for (1), for each $\left(\varphi, \Gamma_{K}\right)$-module $D$ over $\mathbf{B}_{\text {rig, },}^{\dagger}$, Pottharst [32] defined a $\Lambda_{\infty}$-module

$$
\mathbf{H}_{\mathrm{Iw}}^{q}(K, D)
$$

called the analytic Iwasawa cohomology as a generalization of the Iwasawa cohomology of $p$-adic representations. In fact, he proved that we have a functorial $\Lambda_{\infty}$-isomorphism

$$
\mathbf{H}_{\mathrm{IW}}^{q}(K, D(V)) \stackrel{\sim}{\rightarrow} \Lambda_{\infty} \otimes_{\Lambda} \mathbf{H}_{\mathrm{Iw}}^{q}(K, V)
$$

for each $p$-adic representation $V$.

As for $(2)$, let $D$ be a de Rham $(\varphi, \Gamma)$-module. In order to interpolate $\mathbf{D}_{\mathrm{dR}}^{L}(D(k))$, we need to generalize the $\Lambda_{\infty}$-module $\Lambda_{\infty} \otimes_{\mathbb{Q}_{p}} \mathbf{D}_{\text {crys }}^{K}(V)$ for the de Rham case. Our idea is to use Berger's $p$-adic differential equation $\mathbf{N}_{\text {rig }}(D)$. Let $\nabla_{0}:=\frac{\log (\gamma)}{\log \chi(\gamma)} \in \Lambda_{\infty}$, where $\gamma \in \Gamma_{K}$ is a non-torsion element. For each $i \in \mathbb{Z}$, we define $\nabla_{i}:=\nabla_{0}-i \in \Lambda_{\infty}$. Let $\chi: G_{K} \rightarrow \mathbb{Z}_{p}^{\times}$be the $p$-adic cyclotomic character. $\nabla_{0}$ acts on $D$ as a differential operator and acts on $\mathbf{B}_{\text {rig, } \mathbb{Q}_{p}}^{\dagger}$ by $t(1+T) \frac{d}{d T}$. 
In $[3,6]$, for a de Rham $\left(\varphi, \Gamma_{K}\right)$-module $D$ over $\mathbf{B}_{\text {rig, } K}^{\dagger}$, Berger defined a $\left(\varphi, \Gamma_{K}\right)$ submodule $\mathbf{N}_{\text {rig }}(D) \subseteq D[1 / t]$ which satisfies that $\nabla_{0}\left(\mathbf{N}_{\text {rig }}(D)\right) \subseteq t \mathbf{N}_{\text {rig }}(D)$. This condition enables us to define another better differential operator

$$
\tilde{\partial}:=\nabla_{0} \otimes e_{-1}: \mathbf{N}_{\text {rig }}(D) \rightarrow \mathbf{N}_{\text {rig }}(D(-1)) .
$$

The map $\tilde{\partial}$ naturally induces a $\mathbb{Q}_{p}$-linear map

$$
\tilde{\partial}: \mathbf{H}_{\mathrm{Iw}}^{1}\left(K, \mathbf{N}_{\text {rig }}(D)\right) \rightarrow \mathbf{H}_{\mathrm{Iw}}^{1}\left(K, \mathbf{N}_{\text {rig }}(D(-1))\right) .
$$

In $\S 3.2$, we define a canonical projection map for each $L=K, K_{n}$,

$$
T_{L}: \mathbf{H}_{\mathrm{Iw}}^{1}\left(K, \mathbf{N}_{\text {rig }}(D)\right) \rightarrow \mathbf{D}_{\mathrm{dR}}^{L}(D) .
$$

The main theorem of this article is the following (Theorem 3.10), which concerns the existence of a $\Lambda_{\infty}$-morphism $\operatorname{Exp}_{D, h}$ for each $h \in \mathbb{Z}_{\geqq 1}$ such that $\operatorname{Fil}^{-h} \mathbf{D}_{\mathrm{dR}}^{K}(D)=\mathbf{D}_{\mathrm{dR}}^{K}(D)$ which interpolates $\exp _{L, V(k)}$ for some $k \geqq-(h-1)$ and $\exp _{L, D^{\vee}(1-k)}^{*}$ for any $k \leqq-h$.

Theorem 1.5. Let $D$ be a de Rham $\left(\varphi, \Gamma_{K}\right)$-module over $\mathbf{B}_{\text {rig, } K}^{\dagger}$. Let $h \in \mathbb{Z}_{\geqq 1}$ such that $\mathrm{Fil}^{-h} \mathbf{D}_{\mathrm{dR}}^{K}(D)=\mathbf{D}_{\mathrm{dR}}^{K}(D)$. Then there exists a functorial $\Lambda_{\infty}$-linear map

$$
\operatorname{Exp}_{D, h}: \mathbf{H}_{\mathrm{IW}}^{1}\left(K, \mathbf{N}_{\text {rig }}(D)\right) \rightarrow \mathbf{H}_{\mathrm{IW}}^{1}(K, D)
$$

such that, for any $x \in \mathbf{H}_{\mathrm{Iw}}^{1}\left(K, \mathbf{N}_{\text {rig }}(D)\right)$,

(1) if $k \geqq 1$ and there exists $x_{k} \in \mathrm{H}^{1}\left(K, \mathbf{N}_{\text {rig }}(D(k))\right)$ such that $\widetilde{\partial}^{k}\left(x_{k}\right)=x$ or if $0 \geqq k \geqq$ $-(h-1)$ and $x_{k}:=\tilde{\partial}^{-k}(x)$, then

$$
\operatorname{pr}_{L, D(k)}\left(\operatorname{Exp}_{D, h}(x)\right)=\frac{(-1)^{h+k-1}(h+k-1) !\left|\Gamma_{L, \text { tor }}\right|}{p^{m(L)}} \exp _{L, D(k)}\left(T_{L}\left(x_{k}\right)\right)
$$

for each $L=K, K_{n}$,

(2) if $-h \geqq k$, then

$$
\exp _{L, D^{\vee}(1-k)}^{*}\left(\operatorname{pr}_{L, D(k)}\left(\operatorname{Exp}_{D, h}(x)\right)=\frac{\left|\Gamma_{L, \text { tor }}\right|}{(-h-k) ! p^{m(L)}} T_{L}\left(\widetilde{\partial}^{-k}(x)\right)\right.
$$

for each $L=K, K_{n}$,

where we put $m(L):=\min \left\{v_{p}\left(\log (\chi(\gamma)) \mid \gamma \in \Gamma_{L}\right\}\right.$ for each $L=K, K_{n}$.

Remark 1.6. The definition of $\operatorname{Exp}_{D, h}$ is strongly influenced by Berger's work [4] concerning the reinterpretation of the Perrin-Riou map in terms of $(\varphi, \Gamma)$-modules. In particular, this theorem is a generalization of Theorem 2.10 of [4] to all the de Rham $\left(\varphi, \Gamma_{K}\right)$-modules over $\mathbf{B}_{\text {rig, } K}^{\dagger}$ for any $p$-adic field $K$.

Remark 1.7. When $K$ is unramified and $V$ is crystalline, we can easily compare $\Lambda_{\infty} \otimes_{\mathbb{Q}_{p}} \mathbf{D}_{\text {crys }}^{K}(V)$ with $\mathbf{H}_{\mathrm{Iw}}^{1}\left(K, \mathbf{N}_{\text {rig }}(D(V))\right)$. Hence, we can also compare $\Omega_{V, h}$ with $\operatorname{Exp}_{D(V), h}$ by Berger's work above. Therefore, the maps $\operatorname{Exp}_{D, h}$ and their interpolation formulae can be regarded as a generalization of Perrin-Riou's theorem (Theorem 3.2.3 of [29]) on the existence of $\Omega_{V, h}$ and their interpolation formulae to all the de Rham 
$(\varphi, \Gamma)$-modules. Moreover, Pottharst [32] generalized $\Omega_{V, h}$ (precisely, the inverse of $\Omega_{V, h}$ called the big logarithm) to crystalline $(\varphi, \Gamma)$-modules using the theory of Wach modules. We can also compare Pottharst's map with our map. See $\S 3.5$ for more details about the comparison of our big exponential map with their ones in the crystalline case. On the other hand, Colmez (Theorem 7 of [11]) generalized the Perrin-Riou map to all the de Rham $p$-adic representations by a completely different method.

Remark 1.8. In fact, Perrin-Riou and Comez also proved the uniqueness of their big exponential maps using the theory of 'tempered Iwasawa cohomologies'. If we can generalize the theory of tempered Iwasawa cohomologies for $(\varphi, \Gamma)$-modules, it will be possible to prove the uniqueness of our map $\operatorname{Exp}_{D, h}$.

Finally, we prove a theorem (Theorem 3.21) concerning the determinant of $\operatorname{Exp}_{D, h}$. For a torsion co-admissible $\Lambda_{\infty}$-module $M$, denote by $\operatorname{char}_{\Lambda_{\infty}}(M)$ the characteristic ideal of $M$, which is a principal ideal of $\Lambda_{\infty}$.

Theorem 1.9. $(\delta(D))$ Let $D$ be a de Rham $\left(\varphi, \Gamma_{K}\right)$-module over $\mathbf{B}_{\text {rig, } K}^{\dagger}$ of rank $d$ with Hodge-Tate weights $\left\{h_{1}, h_{2}, \ldots, h_{d}\right\}$. For each $h \geqq 1$ such that $\operatorname{Fil}^{-h} \mathbf{D}_{\mathrm{dR}}^{K}(D)=\mathbf{D}_{\mathrm{dR}}^{K}(D)$, we have the following equality of principal fractional ideals of $\Lambda_{\infty}$ :

$$
\begin{aligned}
& \frac{1}{\left(\prod_{i=1}^{d} \prod_{j_{i}=0}^{h-h_{i}-1} \nabla_{h_{i}+j_{i}}\right)^{\left[K: \mathbb{Q}_{p}\right]}} \operatorname{det}_{\Lambda_{\infty}}\left(\mathbf{H}_{\mathrm{IW}}^{1}\left(K, \mathbf{N}_{\mathrm{rig}}(D)\right) \stackrel{\operatorname{Exp}_{D, h}}{\longrightarrow} \mathbf{H}_{\mathrm{IW}}^{1}(K, D)\right) \\
& \quad=\operatorname{char}_{\Lambda_{\infty}}\left(\mathbf{H}_{\mathrm{IW}}^{2}(K, D)\right)\left(\operatorname{char}_{\Lambda_{\infty}} \mathbf{H}_{\mathrm{IW}}^{2}\left(K, \mathbf{N}_{\mathrm{rig}}(D)\right)\right)^{-1} .
\end{aligned}
$$

Remark 1.10. This theorem is a generalization of theorem $\delta(V)$ which was conjectured by Perrin-Riou [29] and was proved as a consequence of her reciprocity law conjecture $\operatorname{Rec}(V)$ proved by Colmez [11], Kato, Kurihara and Tsuji [21], Benois [2] and Berger [4]. Theorem $\delta(V)$ is very important in her works on $p$-adic $L$-functions. For example, it enables us to define the 'inverse of $\Omega_{V, h}$ ', which is a generalization of the Coleman homomorphism and from which we can conjecturally define the $p$-adic $L$-functions associated to $V$. In the non-étale crystalline case, Pottharst also generalized theorem $\delta(V)$ and proved his theorem $\delta(D)$ for all the crystalline $(\varphi, \Gamma)$-modules $D$ by reducing to the étale case using a slope filtration argument. In $\S 3.5$, when $D$ is crystalline, we show that our theorem $\delta(D)$ is equivalent to their theorems $\delta(V)$ or $\delta(D)$. Moreover, our proof does not use $\operatorname{Rec}(V)$ and is via a direct computation rather than by reducing to the étale case, and hence gives a new and more direct proof of their theorems.

Introducing non-étale $(\varphi, \Gamma)$-modules to Iwasawa theory was initiated by Pottharst in $[31,32]$, where he studied Iwasawa's main conjecture for $p$-supersingular modular forms by generalizing the notion of Greenberg's Selmer groups using his theories of the analytic Iwasawa cohomology and of the big logarithm. Our interpolation formula of the big exponential map might help in studying the values of the $p$-adic $L$-functions. Moreover, the author hopes that the results of this article will shed some light on Iwasawa theory or $p$-adic $L$-functions in the case of bad reductions. As another application of this article, in a forthcoming article [27], the author generalize Kato's 
local $\varepsilon$-conjecture [19], which is intimately related with Kato's generalized Iwasawa main conjecture [18], for families of $(\varphi, \Gamma)$-modules over the Robba ring, and prove the conjecture in some special cases using the results of this article.

\section{Notation}

Let $p$ be a prime number. Let $K$ be a finite extension of $\mathbb{Q}_{p}, K_{0}$ the maximal unramified extension of $\mathbb{Q}_{p}$ in $K, \bar{K}$ a fixed algebraic closure of $K$, and $\mathbb{C}_{p}$ the $p$-adic completion of $\bar{K}$. Let $v_{p}: \mathbb{C}_{p}^{\times} \rightarrow \mathbb{Q}$ be the valuation such that $v_{p}(p)=1$. Let $|-|_{p}: \mathbb{C}_{p}^{\times} \rightarrow \mathbb{Q}_{\geqq 0}$ be the $p$-adic absolute value such that $|p|_{p}:=1 / p$. Let $G_{K}:=\mathrm{Gal}(\bar{K} / K)$ be the absolute Galois group of $K$. We fix a set $\left\{\zeta_{p^{n}}\right\}_{n \geqq 1} \subseteq \bar{K}^{\times}$such that $\zeta_{p} \neq 1$ and $\zeta_{p}^{p}=1$ and $\zeta_{p^{n+1}}^{p}=\zeta_{p^{n}}$ for any $n \geqq 1$. We put $K_{n}:=K\left(\zeta_{p^{n}}\right)(n \geqq 1)$ and $K_{\infty}:=\cup_{n \geqq 1} K_{n}$. Let $\chi: G_{K} \rightarrow \mathbb{Z}_{p}^{\times}$be the $p$-adic cyclotomic character (i.e., the character defined by the formula $g\left(\zeta_{p^{n}}\right)=\zeta_{p^{n}}^{\chi(g)}$ for any $n \geqq 1$ and $\left.g \in G_{K}\right)$. We put $\Gamma_{K}:=G_{K} / \operatorname{Ker}(\chi) \stackrel{\sim}{\rightarrow} \operatorname{Gal}\left(K_{\infty} / K\right)$. Denote by the same letter $\chi: \Gamma_{K} \hookrightarrow \mathbb{Z}_{p}^{\times}$the map which is naturally induced by $\chi: G_{K} \rightarrow \mathbb{Z}_{p}^{\times}$. Define the base $e_{1}:=\left(\zeta_{p^{n}}\right)_{n \geqq 1} \in \mathbb{Z}_{p}(1):=\lim _{n} \mu_{p^{n}}(\bar{K})$. Set $e_{k}:=e_{1}^{\otimes k} \in \mathbb{Z}_{p}(k):=\mathbb{Z}_{p}(1)^{\otimes k}$ for each $k \in \mathbb{Z}$. In this article, we normalize the Hodge-Tate weight such that that of $\mathbb{Q}_{p}(1)$ is 1 . For a finite group $G$, we denote by $|G|$ the order of $G$.

\section{The Bloch-Kato exponential map for $(\varphi, \Gamma)$-modules}

In this section, we define the Bloch-Kato exponential map and the dual exponential map for $(\varphi, \Gamma)$-modules over the Robba ring. In $\S 2.1$, we first recall the definition of $(\varphi, \Gamma)$-modules over the Robba ring. In $\S 2.2$, we recall the definitions of some cohomology theories associated to $(\varphi, \Gamma)$-modules. Subsection $\S 2.3$ is the main part of this section, where we generalize Bloch-Kato's fundamental exact sequence to all the $(\varphi, \Gamma)$-modules, and then define the Bloch-Kato exponential map for them and give an explicit formula of this map. In $\S 2.4$, we define the dual exponential map explicitly and then prove that this is the adjoint of our Bloch-Kato exponential map. In $\S 2.5$, we compare our exponential map with the classical Bloch-Kato exponential map using the notion of $B$-pairs.

\section{1. $(\varphi, \Gamma)$-modules over the Robba ring}

In this subsection, we recall the definition of $(\varphi, \Gamma)$-modules over the Robba ring.

We first recall the definition of Fontaine and Berger rings of $p$-adic periods [14, $3]$. Almost all rings in this paragraph are used only in $\S 2.4$, where we compare our exponential map with the classical Bloch-Kato exponential map. Define $\widetilde{\mathbf{E}}^{+}:=$ $\lim _{n \geqq 0} \mathcal{O}_{\mathbb{C}_{p}} / p$, where all the transition maps are the $p$ th power map. The ring $\widetilde{\mathbf{E}}^{+}$ is equipped with a valuation $v_{\widetilde{\mathbf{E}}^{+}}$defined by $v_{\widetilde{\mathbf{E}}^{+}}\left(\left(\bar{x}_{n}\right)_{n \geqq 0}\right):=\lim _{n \rightarrow \infty} p^{n} v_{p}\left(x_{n}\right)$, where $x_{n} \in \mathcal{O}_{\mathbb{C}_{p}}$ is a lift of $\bar{x}_{n} \in \mathcal{O}_{\mathbb{C}_{p}} / p$. By this $v_{\widetilde{\mathbf{E}}^{+}}, \widetilde{\mathbf{E}}^{+}$is a perfect complete valuation ring. We denote by $\widetilde{\mathbf{E}}:=\operatorname{Frac}\left(\widetilde{\mathbf{E}}^{+}\right)$the fraction field of $\widetilde{\mathbf{E}}^{+}$. We define $\varepsilon:=\left(\bar{\zeta}_{p^{n}}\right)_{n \geqq 0} \in \widetilde{\mathbf{E}}^{+}$ for the fixed set $\left\{\zeta_{p^{n}}\right\}_{n \geqq 0}$. We have $v_{\widetilde{\mathbf{E}}^{+}}(\varepsilon-1)=\frac{p}{p-1}$. Define $\widetilde{p}:=\left(\bar{p}_{n}\right)_{n \geqq 0} \in \widetilde{\mathbf{E}}^{+}$where $p_{0}:=p$ and $p_{n+1}^{p}=p_{n}$ for any $n \geqq 0$. Let $\widetilde{\mathbf{A}}^{+}:=W\left(\widetilde{\mathbf{E}}^{+}\right), \widetilde{\mathbf{A}}:=W(\widetilde{\mathbf{E}})$ be the rings of 
Witt vectors of $\widetilde{\mathbf{E}}^{+}$and $\widetilde{\mathbf{E}}$, respectively, which are naturally equipped with actions of $\varphi$ and $G_{K}$. For each $a \in \widetilde{\mathbf{E}}$, denote by $[a] \in \widetilde{\mathbf{A}}$ the Teichmüller lift of $a$. We have a natural $G_{K^{-}}$equivariant surjective ring homomorphism $\theta: \widetilde{\mathbf{A}}^{+} \rightarrow \mathcal{O}_{\mathbb{C}_{p}}$ such that $\theta\left(\left[\left(\bar{x}_{n}\right)_{n \geqq 0}\right]\right):=\lim _{n \rightarrow \infty} x_{n}^{p^{n}}$, where $x_{n} \in \mathcal{O}_{\mathbb{C}_{p}}$ is a lift of $\bar{x}_{n}$. We have $\operatorname{Ker}(\theta)=([\tilde{p}]-p)$. We define $\mathbf{B}_{\mathrm{dR}}^{+}:=\lim _{n \geqq 0} \widetilde{\mathbf{A}}^{+}[1 / p] /(\operatorname{Ker}(\theta)[1 / p])^{n}$ and define an element $t:=\log ([\varepsilon])=$ $\sum_{n \geqq 1} \frac{(-1)^{n-1}}{n}([\varepsilon]-1)^{n} \in \mathbf{B}_{\mathrm{dR}}^{+}$; then $\mathbf{B}_{\mathrm{dR}}^{+}$is a discrete valuation ring with the maximal ideal $t \mathbf{B}_{\mathrm{dR}}^{+}$and with the residue field $\mathbb{C}_{p}$. We have $\varphi(t)=p t$ and $\gamma(t)=\chi(\gamma) t$ for any $\gamma \in \Gamma_{K}$. We put $\mathbf{B}_{\mathrm{dR}}:=\operatorname{Frac}\left(\mathbf{B}_{\mathrm{dR}}^{+}\right)=\mathbf{B}_{\mathrm{dR}}^{+}[1 / t]$. These rings $\mathbf{B}_{\mathrm{dR}}^{+}$and $\mathbf{B}_{\mathrm{dR}}$ are naturally equipped with $G_{K^{-}}$actions. Next, we define $\widetilde{\mathbf{B}}_{\text {rig }}^{\dagger}$ and $\mathbf{B}_{\max }$. For each $0 \leqq r \leqq s<+\infty$ such that $r, s \in \mathbb{Q}$, we define a ring $\widetilde{\mathbf{A}}^{[r, s]}$ as the $p$-adic completion of $\widetilde{\mathbf{A}}^{+}\left[\frac{p}{[\varepsilon-1]^{r}}, \frac{[\varepsilon-1]^{s}}{p}\right]$. We define $\widetilde{\mathbf{B}}^{[r, s]}:=\widetilde{\mathbf{A}}^{[r, s]}[1 / p], \mathbf{B}_{\max }^{+}:=\widetilde{\mathbf{B}}^{\left[0, \frac{p-1}{p}\right]}, \widetilde{\mathbf{B}}_{\text {rig }}^{\dagger, r}:=\cap_{r \leqq s<+\infty} \widetilde{\mathbf{B}}^{[r, s]}$ and $\widetilde{\mathbf{B}}_{\text {rig }}^{\dagger}:=\cup_{r} \widetilde{\mathbf{B}}_{\text {rig }}^{\dagger, r}$

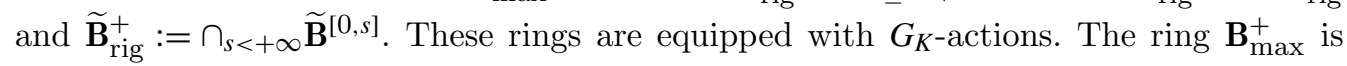
stable by $\varphi$, and $\varphi$ induces isomorphisms $\widetilde{\mathbf{B}}^{[r, s]} \stackrel{\sim}{\rightarrow} \widetilde{\mathbf{B}}^{[p r, p s]}, \widetilde{\mathbf{B}}_{\text {rig }}^{\dagger, r} \stackrel{\sim}{\rightarrow} \widetilde{\mathbf{B}}_{\text {rig }}^{\dagger, p r}$ and $\widetilde{\mathbf{B}}_{\text {rig }}^{\dagger} \stackrel{\sim}{\rightarrow} \widetilde{\mathbf{B}}_{\text {rig }}^{\dagger}$. For each $n \geqq 0$, we put $r_{n}:=p^{n-1}(p-1)=1 / v_{p}\left(\zeta_{p^{n}}-1\right)$. Then, we have a natural injection $\widetilde{\mathbf{B}}^{\left[r_{0}, r_{0}\right]} \hookrightarrow \mathbf{B}_{\mathrm{dR}}^{+}$and a $G_{K^{-}}$equivariant injection $\iota_{n}: \widetilde{\mathbf{B}}_{\mathrm{rig}}^{\dagger, r_{n}} \stackrel{\varphi^{-n}}{\longrightarrow} \widetilde{\mathbf{B}}_{\mathrm{rig}}^{\dagger, r_{0}} \hookrightarrow \widetilde{\mathbf{B}}^{\left[r_{0}, r_{0}\right]} \hookrightarrow \mathbf{B}_{\mathrm{dR}}^{+}$for each $n \geqq 0$. The element $t$ is an element of $\widetilde{\mathbf{B}}_{\text {rig }}^{+}$and, since we have $\widetilde{\mathbf{B}}_{\text {rig }}^{+} \subseteq \mathbf{B}_{\text {max }}^{+}$and $\widetilde{\mathbf{B}}_{\text {rig }}^{+} \subseteq \widetilde{\mathbf{B}}_{\text {rig }}^{\dagger}, t$ is also contained in $\mathbf{B}_{\max }^{+}$and $\widetilde{\mathbf{B}}_{\text {rig }}^{\dagger}$. We define $\mathbf{B}_{\max }:=\mathbf{B}_{\max }^{+}[1 / t]$ and $\mathbf{B}_{e}:=\mathbf{B}_{\max }^{\varphi=1}=\left(\widetilde{\mathbf{B}}_{\text {rig }}^{+}[1 / t]\right)^{\varphi=1}$. One has $\mathbf{B}_{e}=\left(\widetilde{\mathbf{B}}_{\text {rig }}^{\dagger}[1 / t]\right)^{\varphi=1}$ by Lemma 1.1.7 of [5].

We next recall the definition of the Robba ring $\mathbf{B}_{\text {rig, } K}^{\dagger} \subseteq \widetilde{\mathbf{B}}_{\text {rig }}^{\dagger}$. See [3] for more details. We set $T:=[\varepsilon]-1 \in \widetilde{\mathbf{A}}^{+}$. We first assume that $K=F$ is unramified over $\mathbb{Q}_{p}$. For each $r \in \mathbb{Q}_{>0}$, we define a subring $\mathbf{B}_{\text {rig, }, \text { of }}^{\dagger, r} \widetilde{\mathbf{B}}_{\text {rig }}^{\dagger, r}$ by

$$
\mathbf{B}_{\text {rig, }, F}^{\dagger, r}:=\left\{f(T):=\sum_{n \in \mathbb{Z}} a_{n} T^{n} \mid a_{n} \in F \text { and } f(T), \text { is convergent on } p^{-1 / r} \leqq|T|_{p}<1\right\} .
$$

We define $\mathbf{B}_{\text {rig, }, F}^{\dagger}:=\cup_{r>0} \mathbf{B}_{\text {rig, }, F}^{\dagger, r} \subseteq \widetilde{\mathbf{B}}_{\text {rig }}^{\dagger}$. We note that $t=\log (1+T) \in \mathbf{B}_{\text {rig, } \mathbb{Q}_{p}}^{\dagger, r}$ for any $r$. As is a subring of $\widetilde{\mathbf{B}}_{\text {rig }}^{\dagger, r}$, this definition of $\mathbf{B}_{\text {rig, }, F}^{\dagger, r}$ does not depend on the choice of $T$, i.e., does not depend on the choice of $\left\{\zeta_{p^{n}}\right\}_{n \geqq 0}$. For general $K$, we put $F:=K_{0}$ and $e_{K}:=\left[K_{\infty}: F_{\infty}\right]$, and denote by $K_{0}^{\prime} \subseteq K_{\infty}$ the maximal unramified extension of $F$ in $K_{\infty}$. Then, the theory of fields of a norm enables us to define the subring $\mathbf{B}_{\text {rig, } K}^{\dagger}$ of $\widetilde{\mathbf{B}}_{\text {rig }}^{\dagger}$ as a finite étale extension of $\mathbf{B}_{\mathrm{rig}, K_{0}^{\prime}}^{\dagger}$ of degree $e_{K}$, and there exist $r(K) \in \mathbb{Q}_{>0}$ and $\pi_{K} \in \mathbf{B}_{\mathrm{rig}, K}^{\dagger, r(K)}$ such that $\mathbf{B}_{\text {rig, } K}^{\dagger}=\cup_{r \geqq r(K)} \mathbf{B}_{\mathrm{rig}, K}^{\dagger, r}$ is the union of the subrings

$$
\mathbf{B}_{\text {rig, } K}^{\dagger, r}:=\left\{f\left(\pi_{K}\right)=\sum_{n \in \mathbb{Z}} a_{n} \pi_{K}^{n} \mid a_{n} \in F^{\prime} \text { and } f(X) \text { is convergent on } p^{-1 / r e_{K}} \leqq|X|_{p}<1\right\}
$$

of $\widetilde{\mathbf{B}}_{\mathrm{rig}}^{\dagger, r}$. For each finite extension $K \subseteq K^{\prime}(\subseteq \bar{K}), \mathbf{B}_{\mathrm{rig}, K}^{\dagger}$ is a subring of $\mathbf{B}_{\mathrm{rig}, K^{\prime}}^{\dagger}$. The ring $\mathbf{B}_{\text {rig, } K}^{\dagger}$ is stable by the actions of $\varphi$ and $G_{K}$ on $\widetilde{\mathbf{B}}_{\text {rig }}^{\dagger}$. More precisely, we have 
$\varphi\left(\mathbf{B}_{\text {rig, } K}^{\dagger, r}\right) \subseteq \mathbf{B}_{\text {rig, } K}^{\dagger, p r}$, and the action of $G_{K}$ factors through that of $\Gamma_{K}$. When $K=F$ is unramified, then we have an equality $\mathbf{B}_{\text {rig, }, F}^{\dagger}=\mathbf{B}_{\text {rig, } F_{n}}^{\dagger}$ for any $n \geqq 1$, and the actions of $\varphi$ and $\Gamma_{F}$ are explicitly defined by the following formulae, for $f(T)=\sum_{n \in \mathbb{Z}} a_{n} T^{n} \in \mathbf{B}_{\text {rig, },}^{\dagger}$ and $\gamma \in \Gamma_{F}$ :

$$
\varphi(f(T)):=\sum_{n \in \mathbb{Z}} \varphi\left(a_{n}\right)\left((T+1)^{p}-1\right)^{n}, \quad \gamma(f(T)):=\sum_{n \in \mathbb{Z}} a_{n}\left((T+1)^{\chi(\gamma)}-1\right)^{n} .
$$

Next, we define a $\mathbb{Q}_{p}$-linear map $\psi: \mathbf{B}_{\text {rig, } K}^{\dagger} \rightarrow \mathbf{B}_{\text {rig, } K}^{\dagger}$ as follows. It is known that $\mathbf{B}_{\text {rig, } K}^{\dagger}$ can be written as a direct sum $\mathbf{B}_{\text {rig, } K}^{\dagger}=\bigoplus_{i=0}^{p-1}(T+1)^{i} \varphi\left(\mathbf{B}_{\text {rig, } K}^{\dagger}\right)$, so each element $x \in \mathbf{B}_{\text {rig, } K}^{\dagger}$ is uniquely written as $x=\sum_{i=0}^{p-1}(T+1)^{i} \varphi\left(x_{i}\right)$. Then we define $\psi$ by

$$
\psi: \mathbf{B}_{\mathrm{rig}, K}^{\dagger} \rightarrow \mathbf{B}_{\mathrm{rig}, K}^{\dagger}: x=\sum_{i=0}^{p-1}(T+1)^{i} \varphi\left(x_{i}\right) \mapsto x_{0} .
$$

This operator $\psi$ satisfies that $\psi \varphi=$ id and $\psi$ is surjective and commutes with the action of $\Gamma_{K}$. More precisely, if we define $n(K):=\min \left\{n \mid r_{n} \geqq r(K)\right\}$, then we have $\psi\left(\mathbf{B}_{\text {rig, } K}^{\dagger, r_{n+1}}\right)=\mathbf{B}_{\text {rig, }, K}^{\dagger, r_{n}}$ for any $n \geqq n(K)$. For each $n \geqq n(K)$, the restriction of $\iota_{n}: \widetilde{\mathbf{B}}_{\mathrm{rig}}^{\dagger, r_{n}} \hookrightarrow \mathbf{B}_{\mathrm{dR}}^{+}$to $\mathbf{B}_{\mathrm{rig}, K}^{\dagger, r_{n}}$ factors through $K_{n}[[t]] \subseteq \mathbf{B}_{\mathrm{dR}}^{+}$, i.e., $\iota_{n}$ induces a $\Gamma_{K}$-equivariant injection

$$
\iota_{n}: \mathbf{B}_{\mathrm{rig}, K}^{\dagger, r_{n}} \hookrightarrow K_{n}[[t]] .
$$

When $K=F$ is unramified over $\mathbb{Q}_{p}, \iota_{n}: \mathbf{B}_{\mathrm{rig}, F}^{\dagger, r_{n}} \hookrightarrow F_{n}[[t]]$ is explicitly defined by

$$
\iota_{n}\left(\sum_{m \in \mathbb{Z}} a_{m} T^{m}\right):=\sum_{m \in \mathbb{Z}} \varphi^{-n}\left(a_{m}\right)\left(\zeta_{p^{n}} \exp \left(t / p^{n}\right)-1\right)^{m} .
$$

One has the following commutative diagrams:

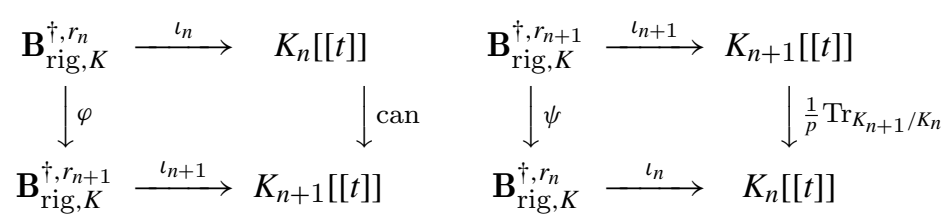

where can : $K_{n}[[t]] \hookrightarrow K_{n+1}[[t]]$ is the canonical injection and $\frac{1}{p} \operatorname{Tr}_{K_{n+1} / K_{n}}$ is defined by

$$
\frac{1}{p} \operatorname{Tr}_{K_{n+1} / K_{n}}: K_{n+1}[[t]] \rightarrow K_{n}[[t]]: \sum_{m=0}^{\infty} a_{m} t^{m} \mapsto \sum_{m=0}^{\infty} \frac{1}{p} \operatorname{Tr}_{K_{n+1} / K_{n}}\left(a_{m}\right) t^{m} .
$$

Definition 2.1. We say that $D$ is a $\left(\varphi, \Gamma_{K}\right)$-module over $\mathbf{B}_{\text {rig, } K}^{\dagger}$ if

(1) $D$ is a finite free $\mathbf{B}_{\text {rig, } K^{-}}^{\dagger}$ module,

(2) $D$ is equipped with a $\varphi$-semi-linear map $\varphi: D \rightarrow D$ such that the linearization map $\varphi^{*}(D):=\mathbf{B}_{\mathrm{rig}, K}^{\dagger} \otimes_{\varphi, \mathbf{B}_{\mathrm{rig}, K}^{\dagger}} D \rightarrow D: a \otimes x \mapsto a \varphi(x)$ is an isomorphism, 
(3) $D$ is equipped with a continuous semi-linear action of $\Gamma_{K}$ which commutes with $\varphi$, where semi-linear means that $\varphi(a x)=\varphi(a) \varphi(x)$ and $\gamma(a x)=\gamma(a) \gamma(x)$ for any $a \in$ $\mathbf{B}_{\text {rig, } K}^{\dagger}, x \in D$ and $\gamma \in \Gamma_{K}$.

Let $D$ be a $\left(\varphi, \Gamma_{K}\right)$-module over $\mathbf{B}_{\text {rig, } K}^{\dagger}$. For each $k$, we denote by $D(k):=D \otimes_{\mathbb{Q}_{p}} \mathbb{Q}_{p}(k)$ the $k$ th Tate twist of $D$. For each finite extension $L$ of $K$, the restriction $\left.D\right|_{L}$ of $D$ to $L$, which is a $\left(\varphi, \Gamma_{L}\right)$-module over $\mathbf{B}_{\text {rig, } L}^{\dagger}$, is defined by

$$
\left.D\right|_{L}:=\mathbf{B}_{\text {rig }, L}^{\dagger} \otimes_{\mathbf{B}_{\text {rig }, K}^{\dagger}} D,
$$

and the actions of $\varphi$ and $\Gamma_{L}\left(\subseteq \Gamma_{K}\right)$ are defined by $\varphi(a \otimes x):=\varphi(a) \otimes \varphi(x), \gamma(a \otimes x):=$ $\gamma(a) \otimes \gamma(x)$ for any $a \in \mathbf{B}_{\text {rig, } L}^{\dagger}, x \in D$ and $\gamma \in \Gamma_{L}$. We define the dual $D^{\vee}$ of $D$ by

$$
D^{\vee}:=\operatorname{Hom}_{\mathbf{B}_{\text {rig }, K}^{\dagger}}\left(D, \mathbf{B}_{\text {rig }, K}^{\dagger}\right),
$$

and, for any $f \in D^{\vee}$ and $\gamma \in \Gamma_{K}, \gamma(f) \in D^{\vee}$ is defined by $\gamma(f)(x):=\gamma\left(f\left(\gamma^{-1} x\right)\right)$ for any $x \in D$, and $\varphi(f) \in D^{\vee}$ is defined by $\varphi(f)\left(\sum_{i=1}^{m} a_{i} \varphi\left(x_{i}\right)\right):=\sum_{i=1}^{m} a_{i} \varphi\left(f\left(x_{i}\right)\right)$ for any $x=\sum_{i=1}^{m} a_{i} \varphi\left(x_{i}\right) \in D\left(a_{i} \in \mathbf{B}_{\text {rig, }, K}^{\dagger}, x_{i} \in D\right)$. Let $D_{1}, D_{2}$ be $\left(\varphi, \Gamma_{K}\right)$-modules over $\mathbf{B}_{\text {rig, }, K}^{\dagger}$. We define the tensor product $D_{1} \otimes D_{2}$ by

$$
D_{1} \otimes D_{2}:=D_{1} \otimes_{\mathbf{B}_{\text {rig }, K}^{\dagger}} D_{2}
$$

with $\varphi$ and $\Gamma_{K}$ acting diagonally. Let $D$ be a $\left(\varphi, \Gamma_{K}\right)$-module over $\mathbf{B}_{\text {rig, } K}^{\dagger}$ of rank $d$. By Theorem 1.3.3 of [6], there exists a $n(D) \geqq n(K)$ and there exists a unique finite free $\mathbf{B}_{\text {rig, }, K}^{\dagger, r_{n(D)}}$-submodule $D^{(n(D))} \subseteq D$ of rank $d$ which satisfies

(1) $\mathbf{B}_{\mathrm{rig}, K}^{\dagger} \otimes_{\mathbf{B}_{\mathrm{rig}, K}^{\dagger, r_{n(D)}}} D^{(n(D))}=D$

(2) if we put $D^{(n)}:=\mathbf{B}_{\mathrm{rig}, K}^{\dagger, r_{n}} \otimes_{\mathbf{B}_{\mathrm{rig}, K}^{\dagger, r_{n(D)}}} D^{(n(D))}$ for each $n \geqq n(D)$, then $\varphi\left(D^{(n)}\right) \subseteq D^{(n+1)}$ and the natural map $\mathbf{B}_{\text {rig, }, K}^{\dagger, r_{n+1}} \otimes_{\varphi, \mathbf{B}_{\text {rig, }, K}^{\dagger, r_{n}}} D^{(n)} \rightarrow D^{(n+1)}: a \otimes x \mapsto a \varphi(x)$ is an isomorphism for any $n \geqq n(D)$.

The uniqueness of $D^{(n)}$ implies that $D^{(n)}$ is preserved by the $\Gamma_{K^{-}}$action for any $n \geqq n(D)$.

Using $D^{(n)}$, we define $\mathbf{D}_{\text {dif }}^{+}(D)$ and $\mathbf{D}_{\text {dif }}(D)$ as follows. For each $n \geqq n(D)$, we put

$$
\mathbf{D}_{\text {dif }, n}^{+}(D):=K_{n}[[t]] \otimes_{\iota_{n}, \mathbf{B}_{\text {rig }, K}^{\dagger, r_{n}}} D^{(n)} \quad\left(\text { respectively } \mathbf{D}_{\text {dif }, n}(D):=K_{n}((t)) \otimes_{K_{n}[[t]]} \mathbf{D}_{\text {dif }, n}^{+}(D)\right),
$$

which is a finite free $K_{n}[[t]]$-module (respectively $K_{n}((t))$-module) of rank $d$ with a semi-linear $\Gamma_{K}$-action. Define a transition map

$$
\mathbf{D}_{\text {dif }, n}^{+}(D) \hookrightarrow \mathbf{D}_{\text {dif }, n+1}^{+}(D): f(t) \otimes x \mapsto f(t) \otimes \varphi(x),
$$

and define a map $\mathbf{D}_{\text {dif }, n}(D) \hookrightarrow \mathbf{D}_{\text {dif }, n+1}(D)$ in the same way. Using these transition maps, we define

$$
\mathbf{D}_{\text {dif }}^{+}(D):=\underset{n}{\lim } \mathbf{D}_{\text {dif }, n}^{+}(D) \quad\left(\text { respectively } \mathbf{D}_{\text {dif }}(D):=\underset{n}{\lim } \mathbf{D}_{\operatorname{dif}, n}(D)\right),
$$


which is a free $K_{\infty}[[t]]:=\cup_{n=1}^{\infty} K_{n}[[t]]$-module (respectively $K_{\infty}((t)):=\cup_{n=1}^{\infty} K_{n}((t)$ )module) of rank $d$ with a semi-linear $\Gamma_{K}$-action. For each $n \geqq n(D)$, define a canonical $\Gamma_{K}$-equivariant injection

$$
\iota_{n}: D^{(n)} \hookrightarrow \mathbf{D}_{\mathrm{dif}, n}^{+}(D): x \mapsto 1 \otimes x
$$

\subsection{Cohomologies of $(\varphi, \Gamma)$-modules}

In this subsection, we recall the definitions of some cohomology theories associated to $(\varphi, \Gamma)$-modules and the fundamental properties of them proved by Liu [23].

Let $\Delta_{K} \subseteq \Gamma_{K}$ be the $p$-torsion subgroup of $\Gamma_{K}$ which is trivial if $p \neq 2$ and at largest cyclic of order 2 if $p=2$. Choose $\gamma_{K} \in \Gamma_{K}$ whose image in $\Gamma_{K} / \Delta_{K}$ is a topological generator (this choice of $\Delta_{K}$ is useful for explicit formulae, but if desired one can reformulate everything to eliminate this choice).

For a $\Delta_{K}$-module $M$, we put $M^{\Delta_{K}}:=\left\{x \in M \mid \gamma^{\prime}(x)=x\right.$ for all $\left.\gamma^{\prime} \in \Delta_{K}\right\}$. For a $\mathbb{Z}\left[\Gamma_{K}\right]$-module $M$, we define a complex $C_{\gamma_{K}}^{\bullet}(M)$ concentrated in degree $[0,1]$ by

$$
C_{\gamma_{K}}^{\bullet}(M):\left[M^{\Delta_{K}} \stackrel{\gamma_{K}-1}{\longrightarrow} M^{\Delta_{K}}\right]
$$

For a $\mathbb{Z}\left[\Gamma_{K}\right]$-module $M$ with a $\varphi$-action which commutes with the action of $\Gamma_{K}$, we define a complex $C_{\varphi, \gamma_{K}}^{\bullet}(M)$ concentrated in degree $[0,2]$ by

$$
C_{\varphi, \gamma_{K}}^{\bullet}(M):\left[M^{\Delta_{K}} \stackrel{d_{1}}{\rightarrow} M^{\Delta_{K}} \oplus M^{\Delta_{K}} \stackrel{d_{2}}{\rightarrow} M^{\Delta_{K}}\right]
$$

with $d_{1}(x):=\left(\left(\gamma_{K}-1\right) x,(\varphi-1) x\right)$ and $d_{2}(x, y):=(\varphi-1) x-\left(\gamma_{K}-1\right) y$.

Let $D$ be a $\left(\varphi, \Gamma_{K}\right)$-module over $\mathbf{B}_{\mathrm{rig}, K}^{\dagger}$. We put $D[1 / t]:=\mathbf{B}_{\mathrm{rig}, K}^{\dagger}[1 / t] \otimes_{\mathbf{B}_{\mathrm{rig}, K}^{\dagger}} D$. For each $q \in \mathbb{Z}_{\geqq 0}$, we define

$$
\left.\mathrm{H}^{q}(K, D):=\mathrm{H}^{q}\left(C_{\varphi, \gamma_{K}}^{\bullet}(D)\right), \quad \mathrm{H}^{q}(K, D[1 / t])\right):=\mathrm{H}^{q}\left(C_{\varphi, \gamma_{K}}^{\bullet}(D[1 / t])\right)
$$

and

$$
\mathrm{H}^{q}\left(K, \mathbf{D}_{\text {dif }}^{+}(D)\right):=\mathrm{H}^{q}\left(C_{\gamma_{K}}^{\bullet}\left(\mathbf{D}_{\text {dif }}^{+}(D)\right)\right), \quad \mathrm{H}^{q}\left(K, \mathbf{D}_{\text {dif }}(D)\right):=\mathrm{H}^{q}\left(C_{\gamma_{K}}^{\bullet}\left(\mathbf{D}_{\text {dif }}(D)\right)\right) .
$$

These definitions are independent of the choice of $\gamma_{K}$. Namely, for each $\gamma_{K}^{\prime} \in \Gamma_{K}$ whose image in $\Gamma_{K} / \Delta_{K}$ is a topological generator, we have $\frac{\gamma_{K}^{\prime}-1}{\gamma_{K}-1} \in \mathbb{Z}_{p}\left[\left[\Gamma_{K} / \Delta_{K}\right]\right]^{\times}$, and we have the canonical isomorphism

$$
\mathrm{H}^{q}\left(C_{\varphi, \gamma_{K}}^{\bullet}(D)\right) \stackrel{\sim}{\rightarrow} \mathrm{H}^{q}\left(C_{\varphi, \gamma_{K}^{\prime}}^{\bullet}(D)\right)
$$

given by the map which is induced by the following map of complexes:

$$
\begin{aligned}
& C_{\varphi, \gamma_{K}}^{\bullet}(D):\left[D^{\Delta_{K}} \stackrel{d_{1}}{\longrightarrow} D^{\Delta_{K}} \oplus D^{\Delta_{K}} \stackrel{d_{2}}{\longrightarrow} D^{\Delta_{K}}\right] \\
& \downarrow \text { id } \quad \downarrow \frac{\gamma_{K}^{\prime}-1}{\gamma_{K}-1} \oplus \text { id } \quad \downarrow \frac{\gamma_{K}^{\prime}-1}{\gamma_{K}-1} \\
& C_{\varphi, \gamma_{K}^{\prime}}^{\bullet}(D):\left[D^{\Delta_{K}} \stackrel{d_{1}}{\longrightarrow} D^{\Delta_{K}} \oplus D^{\Delta_{K}} \stackrel{d_{2}}{\longrightarrow} D^{\Delta_{K}}\right]
\end{aligned}
$$

where we note that the $\mathbb{Z}_{p}\left[\Gamma_{K} / \Delta_{K}\right]$-module structure on $D^{\Delta_{K}}$ uniquely extends to a continuous $\mathbb{Z}_{p}\left[\left[\Gamma_{K} / \Delta_{K}\right]\right]$-module structure. 
For $\left(\varphi, \Gamma_{K}\right)$-modules $D_{1}, D_{2}$ over $\mathbf{B}_{\text {rig, } K}^{\dagger}$, we can define a cup product pairing:

$$
\cup: \mathrm{H}^{q_{1}}\left(K, D_{1}\right) \times \mathrm{H}^{q_{2}}\left(K, D_{2}\right) \rightarrow \mathrm{H}^{q_{1}+q_{2}}\left(K, D_{1} \otimes D_{2}\right) .
$$

See $\S 2.1$ of $[23]$ for the definition. When $\left(q_{1}, q_{2}\right)=(0,1),(1,1)$, the pairing $\cup$ is defined by

$$
\begin{gathered}
\mathrm{H}^{0}\left(K, D_{1}\right) \times \mathrm{H}^{1}\left(K, D_{2}\right) \rightarrow \mathrm{H}^{1}\left(K, D_{1} \otimes D_{2}\right):(a,[x, y]) \mapsto[a \otimes x, a \otimes y], \\
\mathrm{H}^{1}\left(K, D_{1}\right) \times \mathrm{H}^{1}\left(K, D_{2}\right) \rightarrow \mathrm{H}^{2}\left(K, D_{1} \otimes D_{2}\right):\left([x, y],\left[x^{\prime}, y^{\prime}\right]\right) \mapsto\left[y \otimes \varphi\left(x^{\prime}\right)-x \otimes \gamma\left(y^{\prime}\right)\right] .
\end{gathered}
$$

The following theorem was proved by Liu [23] by reducing to the results of Herr $[16,17]$ in the étale case.

Theorem 2.2. Let $D$ be a $\left(\varphi, \Gamma_{K}\right)$-module over $\mathbf{B}_{\mathrm{rig}, K}^{\dagger}$. Then $\mathrm{H}^{q}(K, D)$ satisfies the following.

(0) $\mathrm{H}^{q}(K, D)=0$ if $q \neq 0,1,2$,

(1) for any $q, \mathrm{H}^{q}(K, D)$ is a finite-dimensional $\mathbb{Q}_{p}$-vector space,

(2) $\sum_{q=0}^{2}(-1)^{q} \operatorname{dim}_{\mathbb{Q}_{p}} \mathrm{H}^{q}(K, D)=-\left[K: \mathbb{Q}_{p}\right] \operatorname{rank}(D)$,

(3) we have a canonical isomorphism $f_{\mathrm{tr}}: \mathrm{H}^{2}\left(K, \mathbf{B}_{\mathrm{rig}, K}^{\dagger}(1)\right) \stackrel{\sim}{\rightarrow} \mathbb{Q}_{p}$ and the following pairing $\langle$,$\rangle is perfect for each q=0,1,2$ :

$$
\langle,\rangle: \mathrm{H}^{q}(K, D) \times \mathrm{H}^{2-q}\left(K, D^{\vee}(1)\right) \stackrel{\cup}{\rightarrow} \mathrm{H}^{2}\left(K, D \otimes D^{\vee}(1)\right) \stackrel{\mathrm{ev}}{\rightarrow} \mathrm{H}^{2}\left(K, \mathbf{B}_{\mathrm{rig}, K}^{\dagger}(1)\right) \stackrel{f_{\mathrm{tr}}}{\rightarrow} \mathbb{Q}_{p},
$$

where ev $: \mathrm{H}^{2}\left(K, D \otimes D^{\vee}(1)\right) \stackrel{\mathrm{ev}}{\rightarrow} \mathrm{H}^{2}\left(K, \mathbf{B}_{\mathrm{rig}, K}^{\dagger}(1)\right)$ is the map induced by the evaluation map $D \otimes D^{\vee}(1) \rightarrow \mathbf{B}_{\text {rig }, K}^{\dagger}(1): x \otimes\left(f \otimes e_{1}\right) \mapsto f(x) \otimes e_{1}$.

Proof. See Theorem 0.2 of [23].

Remark 2.3. We remark that Liu proved the existence of functorial comparison isomorphisms $\mathrm{H}^{q}(K, V) \stackrel{\sim}{\rightarrow} \mathrm{H}^{q}(K, D(V))$ for all the $p$-adic representations $V$ of $G_{K}$. Then, the isomorphism $f_{\mathrm{tr}}$ is defined as the composition of the inverse of the comparison isomorphism $\mathrm{H}^{2}\left(K, \mathbb{Q}_{p}(1)\right) \stackrel{\sim}{\rightarrow} \mathrm{H}^{2}\left(K, D\left(\mathbb{Q}_{p}(1)\right)\right)=\mathrm{H}^{2}\left(K, \mathbf{B}_{\text {rig, } K}^{\dagger}(1)\right)$ with Tate's trace $f_{\text {tr }}^{\prime}$ : $\mathrm{H}^{2}\left(K, \mathbb{Q}_{p}(1)\right) \stackrel{\sim}{\rightarrow} \mathbb{Q}_{p}$. In this article, we normalize the isomorphism $f_{\mathrm{tr}}^{\prime}: \mathrm{H}^{2}(K, \mathbb{Q}(1)) \stackrel{\sim}{\rightarrow} \mathbb{Q}_{p}$ such that Tate's pairing

$$
\langle,\rangle: \mathrm{H}^{1}\left(\mathbb{Q}_{p}, \mathbb{Q}_{p}(1)\right) \times \mathrm{H}^{1}\left(\mathbb{Q}_{p}, \mathbb{Q}_{p}\right) \stackrel{\cup}{\rightarrow} \mathrm{H}^{2}\left(\mathbb{Q}_{p}, \mathbb{Q}_{p}(1)\right) \stackrel{f_{\mathrm{tr}}^{\prime}}{\rightarrow} \mathbb{Q}_{p}
$$

satisfies that $\langle\kappa(a), \tau\rangle=\tau\left(\operatorname{rec}_{\mathbb{Q}_{p}}(a)\right)$ for any $a \in \mathbb{Q}_{p}^{\times}$and $\tau \in \operatorname{Hom}\left(G_{\mathbb{Q}_{p}}^{\text {ab }}, \mathbb{Q}_{p}\right)=$ $\mathrm{H}^{1}\left(\mathbb{Q}_{p}, \mathbb{Q}_{p}\right)$, where $\kappa: \mathbb{Q}_{p}^{\times} \rightarrow \mathrm{H}^{1}\left(\mathbb{Q}_{p}, \mathbb{Q}_{p}(1)\right)$ is the Kummer map and $\operatorname{rec}_{\mathbb{Q}_{p}}: \mathbb{Q}_{p}^{\times} \rightarrow G_{\mathbb{Q}_{p}}^{\text {ab }}$ is the reciprocity map of local class field theory.

It is important to define the cohomology $\mathrm{H}^{q}(K, D)$ using $\psi$ instead of $\varphi$, which we recall below. We define a complex $C_{\psi, \gamma_{K}}^{\bullet}(D)$ concentrated in degree $[0,2]$ by

$$
C_{\psi, \gamma_{K}}^{\bullet}(D):\left[D^{\Delta_{K}} \stackrel{d_{1}^{\prime}}{\rightarrow} D^{\Delta_{K}} \oplus D^{\Delta_{K}} \stackrel{d_{2}^{\prime}}{\rightarrow} D^{\Delta_{K}}\right]
$$


with $d_{1}^{\prime}(x):=\left(\left(\gamma_{K}-1\right) x,(\psi-1) x\right)$ and $d_{2}^{\prime}(x, y):=(\psi-1) x-\left(\gamma_{K}-1\right) y$. We define a surjective map $C_{\varphi, \gamma_{K}}^{\bullet}(D) \rightarrow C_{\psi, \gamma_{K}}^{\bullet}(D)$ of complexes by

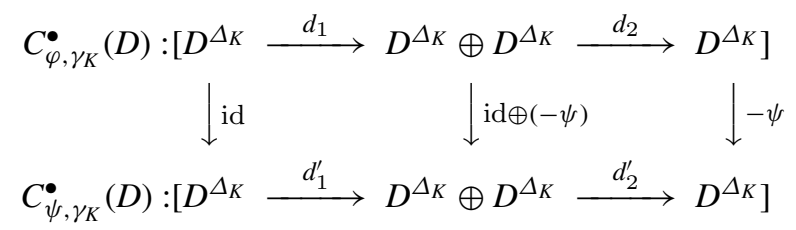

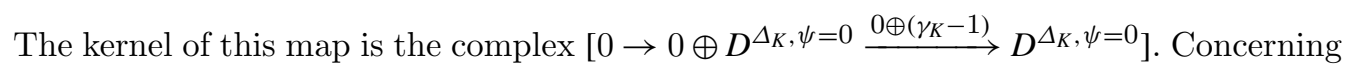
this complex, we have the following theorem.

Theorem 2.4. The map $D^{\Delta_{K}, \psi=0} \stackrel{\gamma_{K}-1}{\longrightarrow} D^{\Delta_{K}, \psi=0}$ is an isomorphism. In particular, the map $C_{\varphi, \gamma_{K}}^{\bullet}(D) \rightarrow C_{\psi, \gamma_{K}}^{\bullet}(D)$ defined above is a quasi-isomorphism.

Proof. For example, see Lemma 2.4 of [23] in the étale case and see Theorem 2.6 of [32] for the general case.

Next, we recall the definition of crystalline or de Rham $(\varphi, \Gamma)$-modules.

Definition 2.5. For a $\left(\varphi, \Gamma_{K}\right)$-module $D$ over $\mathbf{B}_{\text {rig, } K}^{\dagger}$, we define

$$
\mathbf{D}_{\text {crys }}^{K}(D):=D[1 / t]^{\Gamma_{K}=1}, \quad \mathbf{D}_{\mathrm{dR}}^{K}(D):=\mathbf{D}_{\mathrm{dif}}(D)^{\Gamma_{K}=1} .
$$

We define a decreasing filtration on $\mathbf{D}_{\mathrm{dR}}^{K}(D)$ by

$$
\mathrm{Fil}^{i} \mathbf{D}_{\mathrm{dR}}^{K}(D):=\mathbf{D}_{\mathrm{dR}}^{K}(D) \cap t^{i} \mathbf{D}_{\mathrm{dif}}^{+}(D) \subseteq \mathbf{D}_{\mathrm{dif}}(D)
$$

for $i \in \mathbb{Z}$.

Using cohomologies which we defined above, we have equalities

$$
\mathbf{D}_{\mathrm{dR}}^{K}(D)=\mathrm{H}^{0}\left(K, \mathbf{D}_{\mathrm{dif}}(D)\right), \quad \operatorname{Fil}^{0} \mathbf{D}_{\mathrm{dR}}^{K}(D)=\mathrm{H}^{0}\left(K, \mathbf{D}_{\mathrm{dif}}^{+}(D)\right),
$$

and

$$
\mathbf{D}_{\text {crys }}^{K}(D)^{\varphi=1}=\mathrm{H}^{0}(K, D[1 / t]) .
$$

As in the case of $p$-adic Galois representations of $G_{K}$, we have inequalities

$$
\operatorname{dim}_{K_{0}} \mathbf{D}_{\text {crys }}^{K}(D) \leqq \operatorname{dim}_{K} \mathbf{D}_{\mathrm{dR}}^{K}(D) \leqq \operatorname{rank} D
$$

Definition 2.6. Let $D$ be a $\left(\varphi, \Gamma_{K}\right)$-module over $\mathbf{B}_{\text {rig, } K}^{\dagger}$. We say that $D$ is crystalline (respectively de Rham) if an equality $\operatorname{dim}_{K_{0}} \mathbf{D}_{\text {crys }}^{K}(D)=\operatorname{rank}(D)$ (respectively $\left.\operatorname{dim}_{K} \mathbf{D}_{\mathrm{dR}}^{K}(D)=\operatorname{rank}(D)\right)$ holds. We say that $D$ is potentially crystalline if there exists a finite extension $L$ of $K$ such that $\left.D\right|_{L}$ is a crystalline $\left(\varphi, \Gamma_{L}\right)$-module over $\mathbf{B}_{\text {rig, },}^{\dagger}$.

Definition 2.7. Let $D$ be a de $\operatorname{Rham}\left(\varphi, \Gamma_{K}\right)$-module over $\mathbf{B}_{\text {rig, } K}^{\dagger}$. We call the set $\left\{h \in \mathbb{Z} \mid \mathrm{Fil}^{-h} \mathbf{D}_{\mathrm{dR}}^{K}(D) / \mathrm{Fil}^{-h+1} \mathbf{D}_{\mathrm{dR}}^{K}(D) \neq 0\right\}$ the Hodge-Tate weights of $D$.

If $D$ is crystalline then $D$ is also de Rham by the above inequalities. Because potentially de Rham implies de Rham by Hilbert 90, if $D$ is potentially crystalline, then $D$ is de 
Rham. If $D$ is potentially crystalline such that $\left.D\right|_{L}$ is crystalline for a finite Galois extension $L$ of $K$, then $\mathbf{D}_{\text {crys }}^{L}(D):=\mathbf{D}_{\text {crys }}^{L}\left(\left.D\right|_{L}\right)$ is naturally equipped with actions of $\varphi$ and of $\operatorname{Gal}(L / K)$, and we have a natural isomorphism $L \otimes_{L_{0}} \mathbf{D}_{\text {crys }}^{L}(D) \stackrel{\sim}{\rightarrow} \mathbf{D}_{\mathrm{dR}}^{L}(D)=$ $L \otimes_{K} \mathbf{D}_{\mathrm{dR}}^{K}(D)$, i.e., $\mathbf{D}_{\text {crys }}^{L}(D)$ is naturally equipped with a structure of a filtered $(\varphi, \operatorname{Gal}(L / K))$-module.

\subsection{The Bloch-Kato exponential map for $(\varphi, \Gamma)$-modules}

This subsection is the main part of this section. We define a map $\exp _{K, D}: \mathbf{D}_{\mathrm{dR}}^{K}(D) \rightarrow$ $\mathrm{H}^{1}(K, D)$, which is the $(\varphi, \Gamma)$-module analogue of the Bloch-Kato exponential map.

The following is the main theorem of this section, which is the $(\varphi, \Gamma)$-module analogue of the long exact sequence, for a $p$-adic representation $V$ of $G_{K}$,

$$
\begin{aligned}
& 0 \rightarrow \mathrm{H}^{0}(K, V) \rightarrow \mathrm{H}^{0}\left(K, \mathbf{B}_{e} \otimes_{\mathbb{Q}_{p}} V\right) \oplus \mathrm{H}^{0}\left(K, \mathbf{B}_{\mathrm{dR}}^{+} \otimes_{\mathbb{Q}_{p}} V\right) \rightarrow \mathrm{H}^{0}\left(K, \mathbf{B}_{\mathrm{dR}} \otimes_{\mathbb{Q}_{p}} V\right) \\
& \stackrel{\delta_{1, V}}{\longrightarrow} \mathrm{H}^{1}(K, V) \rightarrow \mathrm{H}^{1}\left(K, \mathbf{B}_{e} \otimes_{\mathbb{Q}_{p}} V\right) \oplus \mathrm{H}^{1}\left(K, \mathbf{B}_{\mathrm{dR}}^{+} \otimes_{\mathbb{Q}_{p}} V\right) \rightarrow \mathrm{H}^{1}\left(K, \mathbf{B}_{\mathrm{dR}} \otimes_{\mathbb{Q}_{p}} V\right) \\
& \stackrel{\delta_{2, V}}{\longrightarrow} \mathrm{H}^{2}(K, V) \rightarrow \mathrm{H}^{2}\left(K, \mathbf{B}_{e} \otimes_{\mathbb{Q}_{p}} V\right) \rightarrow 0
\end{aligned}
$$

which is obtained by taking the cohomology long exact sequence associated to the Bloch-Kato fundamental short exact sequence

$$
0 \rightarrow V \rightarrow \mathbf{B}_{e} \otimes_{\mathbb{Q}_{p}} V \oplus \mathbf{B}_{\mathrm{dR}}^{+} \otimes_{\mathbb{Q}_{p}} V \rightarrow \mathbf{B}_{\mathrm{dR}} \otimes_{\mathbb{Q}_{p}} V \rightarrow 0
$$

See $\S 2.5$ for the comparison of the above exact sequence with the following exact sequence.

Theorem 2.8. Let $D$ be a $\left(\varphi, \Gamma_{K}\right)$-module over $\mathbf{B}_{\mathrm{rig}, K}^{\dagger}$. Then there exists a canonical functorial long exact sequence

$$
\begin{aligned}
& 0 \rightarrow \mathrm{H}^{0}(K, D) \rightarrow \mathrm{H}^{0}(K, D[1 / t]) \oplus \mathrm{H}^{0}\left(K, \mathbf{D}_{\text {dif }}^{+}(D)\right) \rightarrow \mathrm{H}^{0}\left(K, \mathbf{D}_{\text {dif }}(D)\right) \\
& \stackrel{\delta_{1, D}}{\longrightarrow} \mathrm{H}^{1}(K, D) \rightarrow \mathrm{H}^{1}(K, D[1 / t]) \oplus \mathrm{H}^{1}\left(K, \mathbf{D}_{\text {dif }}^{+}(D)\right) \rightarrow \mathrm{H}^{1}\left(K, \mathbf{D}_{\text {dif }}(D)\right) \\
& \stackrel{\delta_{2, D}}{\longrightarrow} \mathrm{H}^{2}(K, D) \rightarrow \mathrm{H}^{2}(K, D[1 / t]) \rightarrow 0
\end{aligned}
$$

Proof. To construct this exact sequence, we need to define some more complexes. For each $n \geqq n(D)$, we define a complex with degree in $[0,2]$ :

$$
\tilde{C}_{\varphi, \gamma_{K}}^{\bullet}\left(D^{(n)}\right):\left[\left(D^{(n)}\right)^{\Delta_{K}} \stackrel{d_{1}}{\longrightarrow}\left(D^{(n)}\right)^{\Delta_{K}} \oplus\left(D^{(n+1)}\right)^{\Delta_{K}} \stackrel{d_{2}}{\longrightarrow}\left(D^{(n+1)}\right)^{\Delta_{K}}\right]
$$

with $d_{1}(x):=\left(\left(\gamma_{K}-1\right) x,(\varphi-1) x\right)$ and $d_{2}((x, y)):=(\varphi-1) x-\left(\gamma_{K}-1\right) y$. Define $\tilde{C}_{\varphi, \gamma_{K}}^{\bullet}\left(D^{(n)}[1 / t]\right)$ in the same way. We also define $\tilde{C}_{\varphi, \gamma_{K}}^{\bullet}\left(\mathbf{D}_{\text {dif,n }}^{+}(D)\right)$ with degree in [0.2] by

$$
\left[\prod_{m \geqq n} \mathbf{D}_{\mathrm{dif}, m}^{+}(D)^{\Delta_{K}} \stackrel{d_{1}^{\prime}}{\rightarrow} \prod_{m \geqq n} \mathbf{D}_{\mathrm{dif}, m}^{+}(D)^{\Delta_{K}} \oplus \prod_{m \geqq n+1} \mathbf{D}_{\mathrm{dif}, m}^{+}(D)^{\Delta_{K}} \stackrel{d_{2}^{\prime}}{\rightarrow} \prod_{m \geqq n+1} \mathbf{D}_{\mathrm{dif}, m}^{+}(D)^{\Delta_{K}}\right]
$$

with

$$
d_{1}^{\prime}\left(\left(x_{m}\right)_{m \geqq n}\right):=\left(\left(\left(\gamma_{K}-1\right) x_{m}\right)_{m \geqq n},\left(x_{m-1}-x_{m}\right)_{m \geqq n+1}\right)
$$


and

$$
d_{2}^{\prime}\left(\left(x_{m}\right)_{m \geqq n},\left(y_{m}\right)_{m \geqq n+1}\right):=\left(\left(x_{m-1}-x_{m}\right)-\left(\gamma_{K}-1\right) y_{m}\right)_{m \geqq n+1} .
$$

Define $\tilde{C}_{\varphi, \gamma_{K}}^{\bullet}\left(\mathbf{D}_{\text {dif, } n}(D)\right)=\cup_{k \geqq 0} \tilde{C}_{\varphi, \gamma_{K}}^{\bullet}\left(\frac{1}{t^{k}} \mathbf{D}_{\text {dif }, n}^{+}(D)\right)$. We first prove the following lemma.

Lemma 2.9. Let $D$ be a $\left(\varphi, \Gamma_{K}\right)$-module over $\mathbf{B}_{\mathrm{rig}, K}^{\dagger}$. The following sequence is exact for any $n \geqq n(D)$ :

$$
0 \rightarrow\left(D^{(n)}\right)^{\Delta_{K}} \stackrel{f_{1}}{\rightarrow}\left(D^{(n)}[1 / t]\right)^{\Delta_{K}} \oplus \prod_{m \geqq n} \mathbf{D}_{\mathrm{dif}, m}^{+}(D)^{\Delta_{K}} \stackrel{f_{2}}{\rightarrow} \cup_{k \geqq 0} \prod_{m \geqq n}\left(\frac{1}{t^{k}} \mathbf{D}_{\mathrm{dif}, m}(D)\right)^{\Delta_{K}} \rightarrow 0
$$

where $f_{1}(x):=\left(x,\left(\iota_{m}(x)\right)_{m \geqq n}\right)$ and $f_{2}\left(x,\left(y_{m}\right)_{m \geqq n}\right):=\left(\iota_{m}(x)-y_{m}\right)_{m \geqq n}$.

Proof. Because the functor $M \mapsto M^{\Delta_{K}}$ is exact for $\mathbb{Q}\left[\Delta_{K}\right]$-modules, it suffices to show that the sequence

$$
0 \rightarrow D^{(n)} \stackrel{f_{1}}{\rightarrow} D^{(n)}[1 / t] \oplus \prod_{m \geqq n} \mathbf{D}_{\mathrm{dif}, m}^{+}(D) \stackrel{f_{2}}{\rightarrow} \cup_{k \geqq 0} \frac{1}{t^{k}} \prod_{m \geqq n} \mathbf{D}_{\mathrm{dif}, m}^{+}(D) \rightarrow 0
$$

is exact.

That $f_{1}$ is injective and that $f_{2} \circ f_{1}=0$ are trivial by definition.

If $\left(x,\left(y_{m}\right)_{m \geqq n}\right) \in \operatorname{Ker}\left(f_{2}\right)$, then we have $\iota_{m}(x)=y_{m} \in \mathbf{D}_{\text {dif, } m}^{+}(D)$ for any $m \geqq n$. Hence we have $x \in D^{(n)}$ by Proposition 1.2.2 of [6], and so we have $\left(x,\left(y_{m}\right)_{m \geq n}\right)=f_{1}(x) \in \operatorname{Im}\left(f_{1}\right)$.

Finally, we prove that $f_{2}$ is surjective. Because we have $D^{(n)}[1 / t]=\cup_{k=1}^{\infty} \frac{1}{t^{k}} D^{(n)}$, it suffices to show that the natural map

$$
\frac{1}{t^{k}} D^{(n)} \rightarrow \prod_{m \geqq n} \frac{1}{t^{k}} \mathbf{D}_{\mathrm{dif}, m}^{+}(D) / \mathbf{D}_{\mathrm{dif}, m}^{+}(D): x \mapsto\left(\overline{\iota_{m}(x)}\right)_{m \geqq n}
$$

is surjective for any $k \geqq 1$. Moreover, twisting by $t^{k}$, it suffices to show that the map

$$
D^{(n)} \rightarrow \prod_{m \geqq n} \mathbf{D}_{\mathrm{dif}, m}^{+}(D) / t^{k} \mathbf{D}_{\mathrm{dif}, m}^{+}(D): x \mapsto\left(\overline{\iota_{m}(x)}\right)_{m \geqq n}
$$

is surjective for any $k \geqq 1$. By induction and by dévissage, it suffices to show that this map is surjective for $k=1$. Let $\left\{e_{i}\right\}_{i=1}^{d}$ be a basis of $D$ such that $D^{(n)}=\mathbf{B}_{\text {rig, } K}^{\dagger, r_{n}} e_{1} \oplus \cdots \oplus \mathbf{B}_{\text {rig, }, K}^{\dagger, r_{n}} e_{d}$ for any $n \geqq n(D)$. Then $\left\{\overline{l_{m}\left(e_{i}\right)}\right\}_{i=1}^{d}$ is a $K_{m}$-basis of $\mathbf{D}_{\mathrm{Sen}, m}(D):=\mathbf{D}_{\mathrm{dif}, m}^{+}(D) / t \mathbf{D}_{\mathrm{dif}, m}^{+}(D)$ for any $m \geqq n$ by Lemma 4.9 of [3]. Hence, for any $\left(y_{m}\right)_{m \geqq n} \in \prod_{m \geqq n} \mathbf{D}_{\mathrm{Sen}, m}(D)$, there exists $a_{m, i} \in K_{m} \quad(m \geqq n, 1 \leqq i \leqq d)$ such that $y_{m}=\sum_{i=1}^{d} a_{m, i} \overline{\iota_{m}\left(e_{i}\right)}$ for any $m \geqq n$. Because the natural map $\mathbf{B}_{\text {rig, },}^{\dagger, r_{n}} / t \rightarrow \prod_{m \geqq n} K_{m}$ : $x \mapsto\left(\overline{\iota_{m}(x)}\right)_{m \geqq n}$ is an isomorphism, there exists $\left\{a_{i}\right\}_{1 \leqq i \leqq d} \subseteq \mathbf{B}_{\text {rig, }, K}^{\dagger, r_{n}}$ such that $\overline{\iota_{m}\left(a_{i}\right)}=a_{m, i}$ for any $m \geqq n$ and $i$. Then we have $\overline{\iota_{m}\left(\sum_{i=1}^{d} a_{i} e_{i}\right)}=y_{m}$ for any $m \geqq n$. This proves the surjection of $f_{2}$, and hence proves the lemma.

It is easy to see that the maps $f_{1}, f_{2}$ commute with the differentials of $\tilde{C}_{\varphi, \gamma_{K}}^{\bullet}(-)$. Hence, for each $n \geqq n(D)$, we obtain the following short exact sequence of complexes:

$$
0 \rightarrow \tilde{C}_{\varphi, \gamma_{K}}^{\bullet}\left(D^{(n)}\right) \stackrel{f_{1}}{\rightarrow} \tilde{C}_{\varphi, \gamma_{K}}^{\bullet}\left(D^{(n)}[1 / t]\right) \oplus \tilde{C}_{\varphi, \gamma_{K}}^{\bullet}\left(\mathbf{D}_{\mathrm{dif}, n}^{+}(D)\right) \stackrel{f_{2}}{\rightarrow} \tilde{C}_{\varphi, \gamma_{K}}^{\bullet}\left(\mathbf{D}_{\mathrm{dif}, n}(D)\right) \rightarrow 0 .
$$


We define a transition map

$$
\tilde{C}_{\varphi, \gamma_{K}}^{\bullet}\left(\mathbf{D}_{\mathrm{dif}, n}^{+}\right) \rightarrow \tilde{C}_{\varphi, \gamma_{K}}^{\bullet}\left(\mathbf{D}_{\mathrm{dif}, n+1}^{+}(D)\right)
$$

which is induced by the map

$$
\prod_{m \geqq n} \mathbf{D}_{\text {dif }, m}^{+}(D) \rightarrow \prod_{m \geqq n+1} \mathbf{D}_{\text {dif }, m}^{+}:\left(x_{m}\right)_{m \geqq n} \mapsto\left(x_{m}\right)_{m \geqq n+1} .
$$

We similarly define $\tilde{C}_{\varphi, \gamma_{K}}^{\bullet}\left(\mathbf{D}_{\text {dif }, n}\right) \rightarrow \tilde{C}_{\varphi, \gamma_{K}}^{\bullet}\left(\mathbf{D}_{\text {dif }, n+1}(D)\right)$. Taking the inductive limit with respect to $n \geqq n(D)$, we obtain the following short exact sequence of complexes:

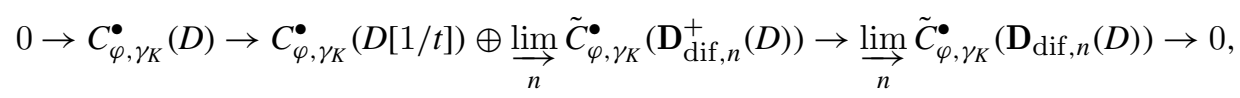

because we have $\lim _{\longrightarrow} \tilde{C}_{\varphi, \gamma_{K}}^{\bullet}\left(D_{1}^{(n)}\right) \stackrel{\sim}{\rightarrow} C_{\varphi, \gamma_{K}}^{\bullet}\left(D_{1}\right)$ for $D_{1}=D, D[1 / t]$. Taking the cohomology long exact sequence, we obtain the following long exact sequence:

$$
\begin{aligned}
& 0 \rightarrow \mathrm{H}^{0}(K, D) \rightarrow \mathrm{H}^{0}(K, D[1 / t]) \oplus \mathrm{H}^{0}\left(\underset{n}{\lim } \tilde{C}_{\varphi, \gamma_{K}}^{\bullet}\left(\mathbf{D}_{\operatorname{dif}, n}^{+}(D)\right)\right) \rightarrow \mathrm{H}^{0}\left(\underset{n}{\lim } \tilde{C}_{\varphi, \gamma_{K}}^{\bullet}\left(\mathbf{D}_{\text {dif }, n}(D)\right)\right) \\
& \stackrel{\delta_{1, D}}{\longrightarrow} \mathrm{H}^{1}(K, D) \rightarrow \mathrm{H}^{1}(K, D[1 / t]) \oplus \mathrm{H}^{1}\left(\underset{\lim }{\longrightarrow} \tilde{C}_{\varphi, \gamma_{K}}^{\bullet}\left(\mathbf{D}_{\mathrm{dif}, n}^{+}(D)\right)\right) \rightarrow \mathrm{H}^{1}\left(\underset{\vec{n}}{\lim } \tilde{C}_{\varphi, \gamma_{K}}^{\bullet}\left(\mathbf{D}_{\operatorname{dif}, n}(D)\right)\right) \\
& \stackrel{\delta_{2, D}}{\longrightarrow} \mathrm{H}^{2}(K, D) \rightarrow \mathrm{H}^{2}(K, D[1 / t]) \oplus \mathrm{H}^{2}\left(\underset{\lim }{\longrightarrow} \tilde{C}_{\varphi, \gamma_{K}}^{\bullet}\left(\mathbf{D}_{\text {dif, },}^{+}(D)\right)\right) \rightarrow \mathrm{H}^{2}\left(\underset{n}{\lim _{n}} \tilde{C}_{\varphi, \gamma_{K}}^{\bullet}\left(\mathbf{D}_{\operatorname{dif}, n}(D)\right)\right) \\
& \rightarrow 0 .
\end{aligned}
$$

Next, for $\mathbf{D}_{\text {dif }, n}^{(+)}(D)=\mathbf{D}_{\text {dif }, n}^{+}(D), \mathbf{D}_{\text {dif }, n}(D)$, define a map of complexes

$$
C_{\gamma_{K}}^{\bullet}\left(\mathbf{D}_{\text {dif }, n}^{(+)}(D)\right) \rightarrow \tilde{C}_{\varphi, \gamma_{K}}^{\bullet}\left(\mathbf{D}_{\text {dif }, n}^{(+)}(D)\right)
$$

by

$$
\begin{gathered}
C_{\gamma_{K}}^{0}\left(\mathbf{D}_{\text {dif }, n}^{(+)}(D)\right) \rightarrow \tilde{C}_{\varphi, \gamma_{K}}^{0}\left(\mathbf{D}_{\text {dif }, n}^{(+)}(D)\right): x \mapsto\left(x_{m}\right)_{m \geqq n} \quad \text { where } x_{m}:=x(m \geqq n), \\
C_{\gamma_{K}}^{1}\left(\mathbf{D}_{\text {dif }, n}^{(+)}(D)\right) \rightarrow \tilde{C}_{\varphi, \gamma_{K}}^{1}\left(\mathbf{D}_{\text {dif }, n}^{(+)}(D)\right): x \mapsto\left(\left(x_{m}\right)_{m \geqq n}, 0\right) \quad \text { where } x_{m}:=x(m \geqq n) .
\end{gathered}
$$

It is easy to check that the map $C_{\gamma_{K}}^{\bullet}\left(\mathbf{D}_{\text {dif }, n}^{(+)}(D)\right) \rightarrow \tilde{C}_{\varphi, \gamma_{K}}^{\bullet}\left(\mathbf{D}_{\text {dif,n }}^{(+)}(D)\right)$ is a quasiisomorphism. Because we have $C_{\gamma_{K}}^{\bullet}\left(\mathbf{D}_{\text {dif }}^{(+)}(D)\right)=\lim _{n} C_{\gamma_{K}}^{\bullet}\left(\mathbf{D}_{\text {dif, },}^{(+)}(D)\right)$, we obtain an isomorphism

$$
\mathrm{H}^{q}\left(K, \mathbf{D}_{\text {dif }}^{(+)}(D)\right) \stackrel{\sim}{\rightarrow} \mathrm{H}^{q}\left(\underset{n}{\lim } \tilde{C}_{\varphi, \gamma_{K}}^{\bullet}\left(\mathbf{D}_{\text {dif }, n}^{(+)}(D)\right)\right) .
$$

Combining the above isomorphisms and the above long exact sequence, we obtain the following desired long exact sequence:

$$
\begin{aligned}
& 0 \rightarrow \mathrm{H}^{0}(K, D) \rightarrow \mathrm{H}^{0}(K, D[1 / t]) \oplus \mathrm{H}^{0}\left(K, \mathbf{D}_{\text {dif }}^{+}(D)\right) \rightarrow \mathrm{H}^{0}\left(K, \mathbf{D}_{\text {dif }}(D)\right) \\
& \stackrel{\delta_{1, D}}{\longrightarrow} \mathrm{H}^{1}(K, D) \rightarrow \mathrm{H}^{1}(K, D[1 / t]) \oplus \mathrm{H}^{1}\left(K, \mathbf{D}_{\mathrm{dif}}^{+}(D)\right) \rightarrow \mathrm{H}^{1}\left(K, \mathbf{D}_{\mathrm{dif}}(D)\right) \\
& \stackrel{\delta_{2, D}}{\longrightarrow} \mathrm{H}^{2}(K, D) \rightarrow \mathrm{H}^{2}(K, D[1 / t]) \rightarrow 0 .
\end{aligned}
$$


The functoriality of this exact sequence is trivial by construction. This finishes the proof of the theorem.

Definition 2.10. Let $D$ be a $\left(\varphi, \Gamma_{K}\right)$-module over $\mathbf{B}_{\text {rig, }, K}^{\dagger}$. Then we define a map

$$
\exp _{K, D}: \mathbf{D}_{\mathrm{dR}}^{K}(D) \rightarrow \mathrm{H}^{1}(K, D)
$$

as the connecting map $\delta_{1, D}: \mathbf{D}_{\mathrm{dR}}^{K}(D)=\mathrm{H}^{0}\left(K, \mathbf{D}_{\text {dif }}(D)\right) \rightarrow \mathrm{H}^{1}(K, D)$ of the long exact sequence of Theorem 2.8. We call $\exp _{K, D}$ the exponential map of $D$.

Remark 2.11. By definition, we have $\exp _{K, D}\left(\mathrm{Fil}^{0} \mathbf{D}_{\mathrm{dR}}(D)\right)=0$. Hence $\exp _{K, D}$ induces a map

$$
\exp _{K, D}: \mathbf{D}_{\mathrm{dR}}^{K}(D) / \mathrm{Fil}^{0} \mathbf{D}_{\mathrm{dR}}^{K}(D) \rightarrow \mathrm{H}^{1}(K, D)
$$

To study the map $\exp _{K, D}$, it is useful to define $\exp _{K, D}$ in a more explicit way. The following lemma gives explicit definitions of $\exp _{K, D}$ and $\delta_{2, D}: \mathrm{H}^{1}\left(K, \mathbf{D}_{\text {dif }}(D)\right) \rightarrow$ $\mathrm{H}^{2}(K, D)$.

Lemma 2.12. (1) Let $x$ be an element of $\mathbf{D}_{\mathrm{dR}}^{K}(D)$. Take an $n \geqq n(D)$ such that $x \in \mathbf{D}_{\mathrm{dif}, n}(D)$, and take an $\tilde{x} \in\left(D^{(n)}[1 / t]\right)^{\Delta_{K}}$ such that $\iota_{m}(\widetilde{x})-x \in \mathbf{D}_{\mathrm{dif}, m}^{+}(D)$ for any $m \geqq n$ (such an $\tilde{x}$ exists by Lemma 2.9). Then we have

$$
\exp _{K, D}(x)=\left[\left(\gamma_{K}-1\right) \widetilde{x},(\varphi-1) \widetilde{x}\right] \in \mathrm{H}^{1}(K, D) .
$$

(2) Let $[x]$ be an element of $\mathrm{H}^{1}\left(K, \mathbf{D}_{\text {dif }}(D)\right)$. Let $x \in \mathbf{D}_{\mathrm{dif}, n}(D)^{\Delta_{K}}$ be a lift of $[x]$ for some $n \geqq n(D)$. Take an $\tilde{x} \in\left(D^{(n)}[1 / t]\right)^{\Delta_{K}}$ such that $\iota_{m}(\tilde{x})-x \in \mathbf{D}_{\mathrm{dif}, m}^{+}(D)$ for any $m \geqq n$ (such an $\widetilde{x}$ exists by Lemma 2.9). Then we have

$$
\delta_{2, D}([x])=[(\varphi-1) \widetilde{x}] \in \mathrm{H}^{2}(K, D) .
$$

Proof. These formulae directly follow from the proof of the theorem above and the construction of the snake lemma.

\subsection{Dual exponential map}

In this subsection, when $D$ is de Rham, we define a map $\exp _{K, D^{\vee}(1)}^{*}: \mathrm{H}^{1}(K, D) \rightarrow$ $\mathrm{Fil}^{0} \mathbf{D}_{\mathrm{dR}}^{K}(D)$, which we call the dual exponential map of $D$. Then, we prove that the map $\exp _{K, D}^{*}: \mathrm{H}^{1}\left(K, D^{\vee}(1)\right) \rightarrow \mathrm{Fil}^{0} \mathbf{D}_{\mathrm{dR}}^{K}\left(D^{\vee}(1)\right)$ is the adjoint of the map $\exp _{K, D}$ : $\mathbf{D}_{\mathrm{dR}}^{K}(D) / \mathrm{Fil}^{0} \mathbf{D}_{\mathrm{dR}}^{K}(D) \rightarrow \mathrm{H}^{1}(K, D)$.

Before defining $\exp _{K, D^{\vee}(1)}^{*}$, we prove some preliminary lemmas. Let $D_{1}, D_{2}$ be $\left(\varphi, \Gamma_{K}\right)$-modules over $\mathbf{B}_{\text {rig, }, K}^{\dagger}$. We define a pairing

$$
\cup_{\text {dif }}: \mathrm{H}^{0}\left(K, \mathbf{D}_{\text {dif }}\left(D_{1}\right)\right) \times \mathrm{H}^{1}\left(K, \mathbf{D}_{\text {dif }}\left(D_{2}\right)\right) \rightarrow \mathrm{H}^{1}\left(K, \mathbf{D}_{\text {dif }}\left(D_{1} \otimes D_{2}\right)\right)
$$

by $x \cup_{\text {dif }}[y]:=[x \otimes y]$ for any $x \in \mathrm{H}^{0}\left(K, \mathbf{D}_{\text {dif }}\left(D_{1}\right)\right)$ and $[y] \in \mathrm{H}^{1}\left(K, \mathbf{D}_{\text {dif }}\left(D_{2}\right)\right)$. 
Lemma 2.13. The following two diagrams are commutative:

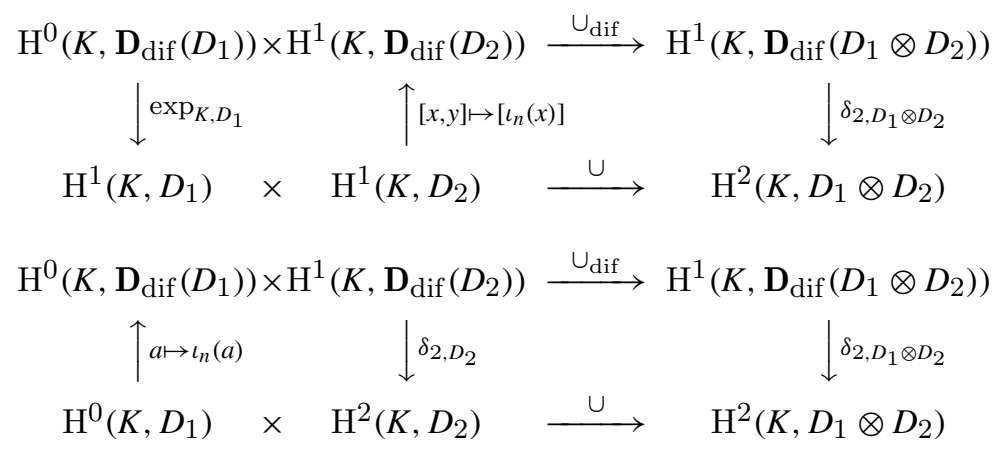

In other words, we have equalities

$$
\delta_{2, D_{1} \otimes D_{2}}\left(z \bigcup_{\text {dif }}\left[\iota_{n}(x)\right]\right)=\exp _{K, D_{1}}(z) \cup[x, y]
$$

and

$$
\delta_{2, D_{1} \otimes D_{2}}\left(\iota_{n}(a) \bigcup_{\text {dif }}[b]\right)=a \cup \delta_{2, D_{2}}([b])
$$

for any $z \in \mathrm{H}^{0}\left(K, \mathbf{D}_{\text {dif }}\left(D_{1}\right)\right),[x, y] \in \mathrm{H}^{1}\left(K, D_{2}\right)$ and $a \in \mathrm{H}^{0}\left(K, D_{1}\right),[b] \in \mathrm{H}^{1}\left(K, \mathbf{D}_{\mathrm{dif}}\left(D_{2}\right)\right)$.

Proof. Here, we only prove the commutativity of the first diagram. We can prove the commutativity of the second diagram in a similar way.

Take any $z \in \mathrm{H}^{0}\left(K, \mathbf{D}_{\text {dif }}\left(D_{1}\right)\right)$ and $[x, y] \in \mathrm{H}^{1}\left(K, D_{2}\right)$. Take $n$ sufficiently large such that $z \in \mathbf{D}_{\text {dif, }, n}\left(D_{1}\right)$ and $x \in D_{2}^{(n)}, y \in D_{2}^{(n+1)}$. By Lemma 2.12(1), if we take $\tilde{z} \in\left(D_{1}^{(n)}[1 / t]\right)^{\Delta_{K}}$ such that $\iota_{m}(\tilde{z})-z \in \mathbf{D}_{\mathrm{dif}, m}^{+}\left(D_{1}\right)$ for any $m \geqq n$, then we have

$$
\exp _{K, D_{1}}(z)=\left[\left(\gamma_{K}-1\right) \tilde{z},(\varphi-1) \tilde{z}\right]
$$

Hence, we have

$$
\begin{aligned}
\exp _{K, D_{1}}(z) \cup[x, y] & =\left[\left(\gamma_{K}-1\right) \tilde{z},(\varphi-1) \tilde{z}\right] \cup[x, y] \\
& =\left[(\varphi-1) \tilde{z} \otimes \varphi(x)-\left(\gamma_{K}-1\right) \tilde{z} \otimes \gamma_{K}(y)\right] \in \mathrm{H}^{2}\left(K, D_{1} \otimes D_{2}\right) .
\end{aligned}
$$

On the other hand, under the natural quasi-isomorphism $C_{\gamma_{K}}^{\bullet}\left(\mathbf{D}_{\text {dif }, n}(D)\right) \rightarrow$ $\tilde{C}_{\varphi, \gamma_{K}}^{\bullet}\left(\mathbf{D}_{\text {dif }, n}(D)\right)$ which is defined in the proof of Theorem 2.8 , the element $\left[\iota_{n}(x)\right] \in$ $\mathrm{H}^{1}\left(K, \mathbf{D}_{\text {dif }, n}\left(D_{2}\right)\right)$ is sent to

$$
\left[\left(\iota_{m}(x)\right)_{m \geqq n},\left(\iota_{m}(y)\right)_{m \geqq n+1}\right] \in \mathrm{H}^{1}\left(\tilde{C}_{\varphi, \gamma_{K}}^{\bullet}\left(\mathbf{D}_{\mathrm{dif}, n}\left(D_{2}\right)\right)\right) .
$$

Hence, the element $z \cup_{\text {dif }}\left[\iota_{n}(x)\right] \in \mathrm{H}^{1}\left(K, \mathbf{D}_{\text {dif }, n}\left(D_{1} \otimes D_{2}\right)\right)$ is sent to

$$
\left[\left(z \otimes \iota_{m}(x)\right)_{m \geqq n},\left(z \otimes \iota_{m}(y)\right)_{m \geqq n+1}\right] \in \mathrm{H}^{1}\left(\tilde{C}_{\varphi, \gamma_{K}}^{\bullet}\left(\mathbf{D}_{\text {dif }, n}\left(D_{1} \otimes D_{2}\right)\right)\right) .
$$

The element $(\tilde{z} \otimes x, \tilde{z} \otimes y) \in\left(D_{1}^{(n)} \otimes D_{2}^{(n)}[1 / t]\right)^{\Delta_{K}} \bigoplus\left(D_{1}^{(n+1)} \otimes D_{2}^{(n+1)}[1 / t]\right)^{\Delta_{K}} \quad$ satisfies that $\left(\left(\iota_{m}(\tilde{z} \otimes x)\right)_{m \geqq n},\left(\iota_{m}(\tilde{z} \otimes y)\right)_{m \geqq n+1}\right)-\left(\left(z \otimes \iota_{m}(x)\right)_{m \geqq n},\left(z \otimes \iota_{m}(y)\right)_{m \geqq n+1}\right) \in \prod_{m \geqq n} \mathbf{D}_{\text {dif, } m}^{+}$ 
$\left(D_{1} \otimes D_{2}\right) \bigoplus \prod_{m \geqq n+1} \mathbf{D}_{\text {dif }, m}^{+}\left(D_{1} \otimes D_{2}\right)$. Hence, by the definition of the boundary map $\delta_{2, D_{1} \otimes D_{2}}$, we obtain

$$
\delta_{2, D_{1} \otimes D_{2}}\left(z \bigcup_{\text {dif }}\left[\iota_{n}(x)\right]\right)=\left[(\varphi-1)(\tilde{z} \otimes x)-\left(\gamma_{K}-1\right)(\tilde{z} \otimes y)\right] .
$$

Using the equality $(\varphi-1) x=\left(\gamma_{K}-1\right) y$, it is easy to show the equality

$$
(\varphi-1) \tilde{z} \otimes \varphi(x)-\left(\gamma_{K}-1\right) \tilde{z} \otimes \gamma_{K}(y)=(\varphi-1)(\tilde{z} \otimes x)-\left(\gamma_{K}-1\right)(\tilde{z} \otimes y) .
$$

Hence we obtain the desired equality

$$
\exp _{K, D_{1}}(z) \cup[x, y]=\delta_{2, D_{1} \otimes D_{2}}\left(z \bigcup_{\text {dif }}\left[\iota_{n}(x)\right]\right) .
$$

Let $D$ be a de Rham $\left(\varphi, \Gamma_{K}\right)$-module over $\mathbf{B}_{\text {rig, } K}^{\dagger}$. Then the natural map

$$
K_{\infty}((t)) \otimes_{K} \mathbf{D}_{\mathrm{dR}}^{K}(D) \rightarrow \mathbf{D}_{\mathrm{dif}}(D): f(t) \otimes x \mapsto f(t) x
$$

is an isomorphism. We identify $K_{\infty}((t)) \otimes_{K} \mathbf{D}_{\mathrm{dR}}^{K}(D)$ with $\mathbf{D}_{\text {dif }}(D)$ by this isomorphism. Then, it is easy to check that the map

$$
g_{D}: \mathbf{D}_{\mathrm{dR}}^{K}(D) \stackrel{\sim}{\rightarrow} \mathrm{H}^{1}\left(K, \mathbf{D}_{\mathrm{dif}}(D)\right)\left(\stackrel{\sim}{\rightarrow} \mathrm{H}^{1}\left(C_{\gamma_{K}}^{\bullet}\left(K_{\infty}((t)) \otimes_{K} \mathbf{D}_{\mathrm{dR}}^{K}(D)\right)\right)\right)
$$

defined by

$$
g_{D}(x):=\left[\log \left(\chi\left(\gamma_{K}\right)\right)(1 \otimes x)\right] \in \mathrm{H}^{1}\left(C_{\gamma_{K}}^{\bullet}\left(K_{\infty}((t)) \otimes_{K} \mathbf{D}_{\mathrm{dR}}^{K}(D)\right)\right)
$$

is an isomorphism and is independent of the choice of $\gamma_{K}$ up to the canonical isomorphism. We note that $\mathbf{D}_{\mathrm{dR}}^{K}\left(\mathbf{B}_{\mathrm{rig}, K}^{\dagger}(1)\right)=K \cdot \frac{1}{t} e_{1}$.

Lemma 2.14. The following diagram is commutative:

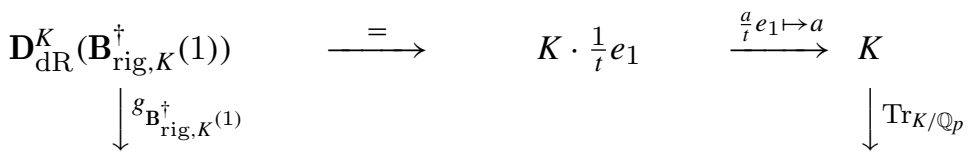

$$
\begin{aligned}
& \mathrm{H}^{1}\left(K, \mathbf{D}_{\text {dif }}\left(\mathbf{B}_{\text {rig }, K}^{\dagger}(1)\right)\right) \stackrel{\delta_{2, \mathbf{B}_{\text {rig }, K}^{\dagger}}}{\longrightarrow} \mathrm{H}^{2}\left(K, \mathbf{B}_{\text {rig }, K}^{\dagger}(1)\right) \stackrel{f_{\mathrm{tr}}}{\longrightarrow} \mathbb{Q}_{p}
\end{aligned}
$$

Proof. Using the trace and the corestriction, it suffices to show the lemma when $K=\mathbb{Q}_{p}$. Assume that $K=\mathbb{Q}_{p}$. We prove the lemma by comparing the cohomology of the $\left(\varphi, \Gamma_{\mathbb{Q}_{p}}\right)$-module $\mathbf{B}_{\text {rig, } \mathbb{Q}_{p}}^{\dagger}(1)$ with the Galois cohomology of the $p$-adic representation $\mathbb{Q}_{p}(1)$ of $G_{\mathbb{Q}_{p}}$ as follows. Please see $\S 2.5$ for some notation and definitions used in the proof below.

Take the element $\left[\log \left(\chi\left(\gamma_{K}\right)\right), 0\right] \in \mathrm{H}^{1}\left(\mathbb{Q}_{p}, \mathbf{B}_{\text {rig, } \mathbb{Q}_{p}}^{\dagger}\right)$. Then we have

$$
g_{\mathbf{B}_{\mathrm{rig}, \mathbb{Q}_{p}}^{\dagger}(1)}\left(\frac{a}{t} e_{1}\right)=\frac{a}{t} e_{1} \bigcup_{\mathrm{dif}}\left[\iota_{n}\left(\log \left(\chi\left(\gamma_{K}\right)\right)\right)\right]
$$


by the definition of $g_{D}$ and $\cup_{\text {dif }}$. Hence, by Lemma 2.13 , we obtain an equality

$$
\begin{aligned}
\delta_{2, \mathbf{B}_{\mathrm{rig}, \mathbb{Q}_{p}}^{\dagger}(1)}\left(g_{\mathbf{B}_{\mathrm{rig}, \mathbb{Q}_{p}}^{\dagger}(1)}\left(\frac{a}{t} e_{1}\right)\right) & =\delta_{2, \mathbf{B}_{\mathrm{rig}, \mathbb{Q}_{p}}^{\dagger}(1)}\left(\frac{a}{t} e_{1} \bigcup_{\operatorname{dif}}\left[\iota_{n}\left(\log \left(\chi\left(\gamma_{K}\right)\right)\right)\right]\right) \\
& =\exp _{\mathbb{Q}_{p}, \mathbf{B}_{\mathrm{rig}, \mathbb{Q} p}^{\dagger}(1)}\left(\frac{a}{t} e_{1}\right) \cup\left[\log \left(\chi\left(\gamma_{K}\right)\right), 0\right]
\end{aligned}
$$

for any $a \in \mathbb{Q}_{p}$. Hence, it suffices to show that

$$
\left.f_{\operatorname{tr}}\left(\exp _{\mathbb{Q}_{p}, \mathbf{B}_{\mathrm{rig}, \mathbb{Q}_{p}}^{\dagger}(1)}\left(\frac{a}{t} e_{1}\right)\right) \cup\left[\log \left(\chi\left(\gamma_{K}\right)\right), 0\right]\right)=a .
$$

We note that the comparison isomorphism

$$
\mathrm{H}^{1}\left(\mathbb{Q}_{p}, \mathbf{B}_{\mathrm{rig}, \mathbb{Q}_{p}}^{\dagger}\right) \stackrel{\sim}{\rightarrow} \mathrm{H}^{1}\left(\mathbb{Q}_{p}, W\left(\mathbf{B}_{\mathrm{rig}, \mathbb{Q}_{p}}^{\dagger}\right) \stackrel{\sim}{\rightarrow} \mathrm{H}^{1}\left(\mathbb{Q}_{p}, \mathbb{Q}_{p}\right)\right.
$$

(where $\mathbb{Q}_{p}$ on the right-hand side is the trivial $p$-adic representation of $G_{\mathbb{Q}_{p}}$ ) sends $\left[\log \left(\chi\left(\gamma_{K}\right)\right), 0\right]$ to the element $\log (\chi) \in \operatorname{Hom}\left(G_{\mathbb{Q}_{p}}^{\mathrm{ab}}, \mathbb{Q}_{p}\right)=\mathrm{H}^{1}\left(\mathbb{Q}_{p}, \mathbb{Q}_{p}\right)$ defined by $\log (\chi)$ : $G_{\mathbb{Q}_{p}}^{\mathrm{ab}} \rightarrow \mathbb{Q}_{p}: g \mapsto \log (\chi(g))$. Hence, by Theorem 2.21 , in particular, by the compatibility of $\exp _{K, D}$ with $\exp _{K, W(D)}$ (we remark that we do not use any results in this subsection $(\S 2.4)$ to prove this compatibility, but we use them to prove the compatibility of $\delta_{2, D}$ with $\left.\delta_{2, W(D)}\right)$, it suffices to show that Tate's pairing satisfies

$$
\left\langle\exp _{\mathbb{Q}_{p}, \mathbb{Q}_{p}}\left(\frac{a}{t} e_{1}\right), \log (\chi)\right\rangle=a
$$

for any $a \in \mathbb{Q}_{p}$. Because it is known that $\kappa(b)=\exp _{\mathbb{Q}_{p}, \mathbb{Q}_{p}}\left(\frac{\log (b)}{t} e_{1}\right)$ for any $b \in \mathbb{Z}_{p}^{\times}$ $\left(\kappa: \mathbb{Q}_{p}^{\times} \rightarrow \mathrm{H}^{1}\left(\mathbb{Q}_{p}, \mathbb{Q}_{p}(1)\right)\right.$ is the Kummer map), we obtain

$$
\begin{aligned}
\left\langle\exp _{\mathbb{Q}_{p}, \mathbb{Q}_{p}}\left(\frac{\log (b)}{t} e_{1}\right), \log (\chi)\right\rangle & =\langle\kappa(b), \log (\chi)\rangle \\
& =\log (b)
\end{aligned}
$$

for any $b \in \mathbb{Z}_{p}^{\times}$, where the last equality follows from Remark 2.3. This finishes the proof of the lemma.

Let $D$ be a $\left(\varphi, \Gamma_{K}\right)$-module over $\mathbf{B}_{\text {rig, } K}^{\dagger}$. We define a pairing

$$
\begin{aligned}
\langle,\rangle_{\text {dif }}: & \mathrm{H}^{0}\left(K, \mathbf{D}_{\text {dif }}(D)\right) \times \mathrm{H}^{1}\left(K, \mathbf{D}_{\text {dif }}\left(D^{\vee}(1)\right)\right) \stackrel{\cup_{\text {dif }}}{\longrightarrow} \mathrm{H}^{1}\left(K, \mathbf{D}_{\text {dif }}\left(D \otimes D^{\vee}(1)\right)\right) \\
& \stackrel{\text { ev }}{\longrightarrow} \mathrm{H}^{1}\left(K, \mathbf{D}_{\text {dif }}\left(\mathbf{B}_{\mathrm{rig}, K}^{\dagger}(1)\right)\right) \stackrel{\mathbf{B}_{\mathrm{rig}, K}^{\dagger}}{\longrightarrow} \mathbf{D}_{\mathrm{dR}}^{K}\left(\mathbf{B}_{\mathrm{rig}, K}^{\dagger}(1)\right) \stackrel{\frac{a}{t} e_{1} \mapsto a}{\longrightarrow} K .
\end{aligned}
$$

Lemma 2.15. Let $D$ be a $\left(\varphi, \Gamma_{K}\right)$-module over $\mathbf{B}_{\mathrm{rig}, K}^{\dagger}$. Then the following diagrams

$$
\begin{aligned}
& \mathrm{H}^{0}\left(K, \mathbf{D}_{\text {dif }}(D)\right) \times \mathrm{H}^{1}\left(K, \mathbf{D}_{\text {dif }}\left(D^{\vee}(1)\right)\right) \stackrel{\langle,\rangle_{\text {dif }}}{\longrightarrow} K \\
& \downarrow \exp _{K, D} \quad \uparrow[x, y] \mapsto\left[\iota_{n}(x)\right] \quad \downarrow \operatorname{Tr}_{K / \mathbb{Q} p} \\
& \mathrm{H}^{1}(K, D) \quad \times \quad \mathrm{H}^{1}\left(K, D^{\vee}(1)\right) \underset{\langle,\rangle}{\longrightarrow} \mathbb{Q}_{p}
\end{aligned}
$$


and

$$
\begin{array}{rrrrr}
\mathrm{H}^{0}\left(K, \mathbf{D}_{\text {dif }}(D)\right) \times \mathrm{H}^{1}\left(K, \mathbf{D}_{\text {dif }}\left(D^{\vee}(1)\right)\right) & \stackrel{\langle,\rangle_{\text {dif }}}{\longrightarrow} & K \\
\uparrow_{x \mapsto l_{n}(x)} & \downarrow \delta_{2, D^{\vee}(1)} & & & \downarrow \operatorname{Tr}_{K / \mathbb{Q} p} \\
\mathrm{H}^{0}(K, D) & \times & \mathrm{H}^{2}\left(K, D^{\vee}(1)\right) & \longrightarrow,\rangle & \mathbb{Q}_{p}
\end{array}
$$

are commutative. In other words, we have equalities

$$
\left\langle\exp _{K, D}(z),[x, y]\right\rangle=\operatorname{Tr}_{K / \mathbb{Q}_{p}}\left(\left\langle z,\left[\iota_{n}(x)\right]\right\rangle_{\text {dif }}\right)
$$

and

$$
\left\langle a, \delta_{2, D^{\vee}(1)}([b])\right\rangle=\operatorname{Tr}_{K / \mathbb{Q}_{p}}\left(\left\langle\iota_{n}(a),[b]\right\rangle_{\text {dif }}\right)
$$

for any $z \in \mathrm{H}^{0}\left(K, \mathbf{D}_{\text {dif }}(D)\right),[x, y] \in \mathrm{H}^{1}\left(K, D^{\vee}(1)\right) \quad$ and $\quad a \in \mathrm{H}^{0}(K, D), \quad[b] \in \mathrm{H}^{1}(K$, $\left.\mathbf{D}_{\text {dif }}\left(D^{\vee}(1)\right)\right)$.

Proof. This lemma follows from Lemmas 2.13 and 2.14 .

Let $D$ be a de Rham $\left(\varphi, \Gamma_{K}\right)$-module over $\mathbf{B}_{\text {rig, } K}^{\dagger}$.

We define a map

$$
\exp _{K, D^{\vee}(1)}^{*}: \mathrm{H}^{1}(K, D) \rightarrow \operatorname{Fil}^{0} \mathbf{D}_{\mathrm{dR}}^{K}(D)
$$

as the composition of the natural map

$$
\mathrm{H}^{1}(K, D) \rightarrow \mathrm{H}^{1}\left(K, \mathbf{D}_{\text {dif }}^{+}(D)\right) \rightarrow \mathrm{H}^{1}\left(K, \mathbf{D}_{\text {dif }}(D)\right):[x, y] \mapsto\left[\iota_{n}(x)\right]
$$

(for sufficiently large $n$ ) with the inverse of the isomorphism

$$
g_{D}: \mathbf{D}_{\mathrm{dR}}^{K}(D) \stackrel{\sim}{\rightarrow} \mathrm{H}^{1}\left(K, \mathbf{D}_{\mathrm{dif}}(D)\right) .
$$

Because we have $\mathbf{D}_{\text {dif }}^{+}(D)=\operatorname{Fil}^{0}\left(K_{\infty}((t)) \otimes_{K} \mathbf{D}_{\mathrm{dR}}^{K}(D)\right)$, we can easily see that the image of $\exp _{K, D^{\vee}(1)}^{*}$ is contained in $\mathrm{Fil}^{0} \mathbf{D}_{\mathrm{dR}}^{K}(D)$. As in the case of $p$-adic Galois representations, the map $\exp _{K, D}^{*}$ is the adjoint of $\exp _{K, D}$ in the following sense. We define a $K$-bi-linear perfect pairing $[-,-]_{\mathrm{dR}}$ by

$$
[-,-]_{\mathrm{dR}}: \mathbf{D}_{\mathrm{dR}}^{K}(D) \times \mathbf{D}_{\mathrm{dR}}^{K}\left(D^{\vee}(1)\right) \stackrel{\mathrm{ev}}{\longrightarrow} \mathbf{D}_{\mathrm{dR}}^{K}\left(\mathbf{B}_{\mathrm{rig}, K}^{\dagger}(1)\right) \stackrel{\frac{a}{t} e_{1} \mapsto a}{\longrightarrow}, K
$$

where ev is the natural evaluation map. By the definition of $\mathrm{Fil}^{i}$, this pairing induces a perfect pairing

$$
[-,-]_{\mathrm{dR}}: \mathbf{D}_{\mathrm{dR}}^{K}(D) / \mathrm{Fil}^{0} \mathbf{D}_{\mathrm{dR}}^{K}(D) \times \mathrm{Fil}^{0} \mathbf{D}_{\mathrm{dR}}^{K}\left(D^{\vee}(1)\right) \rightarrow K .
$$

Proposition 2.16. Let $D$ be a de Rham $\left(\varphi, \Gamma_{K}\right)$-module over $\mathbf{B}_{\mathrm{rig}, K}^{\dagger}$. For any $\bar{x} \in$ $\mathbf{D}_{\mathrm{dR}}^{K}(D) / \mathrm{Fil}^{0} \mathbf{D}_{\mathrm{dR}}^{K}(D)$ and $y \in \mathrm{H}^{1}\left(K, D^{\vee}(1)\right)$, the following equality holds:

$$
\left\langle\exp _{K, D}(\bar{x}), y\right\rangle=\operatorname{Tr}_{K / \mathbb{Q}_{p}}\left(\left[\bar{x}, \exp _{K, D}^{*}(y)\right]_{\mathrm{dR}}\right) .
$$

Proof. By Lemma 2.15, it suffices to show the equality

$$
[x, z]_{\mathrm{dR}}=\left\langle x, g_{D^{\vee}(1)}(z)\right\rangle_{\mathrm{dif}}
$$


for any $x \in \mathrm{H}^{0}\left(K, \mathbf{D}_{\text {dif }}(D)\right), z \in \mathrm{H}^{0}\left(K, \mathbf{D}_{\text {dif }}\left(D^{\vee}(1)\right)\right)$; but this equality is trivial by definition.

\subsection{Comparison with the Bloch-Kato exponential map of $B$-pairs}

In this subsection, we show that the long exact sequence of Theorem 2.8 associated to $D$ is isomorphic to the long exact sequence naturally defined from the cohomologies of the corresponding $B$-pair $W(D)$. In particular, in the étale case, we show that the sequence of Theorem 2.8 is isomorphic to the long exact sequence induced from the Bloch-Kato fundamental short exact sequence.

We first recall the definition of $B$-pairs and the definition of the functor from the category of $(\varphi, \Gamma)$-modules to the category of $B$-pairs which induces an equivalence between these categories; see [5] for more details.

The following definition is due to Berger [5].

Definition 2.17. We say that a pair $W:=\left(W_{e}, W_{\mathrm{dR}}^{+}\right)$is a $B$-pair of $G_{K}$ if

(1) $W_{e}$ is a finite free $\mathbf{B}_{e}$-module with a continuous semi-linear $G_{K}$-action,

(2) $W_{\mathrm{dR}}^{+}$is a $G_{K}$-stable finite $\mathbf{B}_{\mathrm{dR}}^{+}$-submodule of $W_{\mathrm{dR}}:=\mathbf{B}_{\mathrm{dR}} \otimes_{\mathbf{B}_{e}} W_{e}$ which generates $W_{\mathrm{dR}}$ as $\mathbf{B}_{\mathrm{dR}}$-module,

where semi-linear means that $g(a x)=g(a) g(x)$ for any $a \in \mathbf{B}_{e}, x \in W_{e}$ and $g \in G_{K}$.

Remark 2.18. Let $V$ be a $p$-adic representation of $G_{K}$. We define a $B$-pair

$$
W(V):=\left(\mathbf{B}_{e} \otimes_{\mathbb{Q}_{p}} V, \mathbf{B}_{\mathrm{dR}}^{+} \otimes_{\mathbb{Q}_{p}} V\right) .
$$

By Bloch-Kato's fundamental short exact sequence [7]

$$
0 \rightarrow \mathbb{Q}_{p} \stackrel{x \mapsto(x, x)}{\longrightarrow} \mathbf{B}_{e} \bigoplus \mathbf{B}_{\mathrm{dR}}^{+} \stackrel{(x, y) \mapsto x-y}{\longrightarrow} \mathbf{B}_{\mathrm{dR}} \rightarrow 0,
$$

we can easily see that this functor $V \mapsto W(V)$ is fully faithful; hence we can view the category of $p$-adic representations of $G_{K}$ as a full subcategory of the category of $B$-pairs of $G_{K}$.

By the theorems of Fontaine, Cherbonnier and Colmez and Kedlaya, the category of $p$-adic representations of $G_{K}$ is equivalent to the category of étale $\left(\varphi, \Gamma_{K}\right)$-modules over $\mathbf{B}_{\text {rig, } K}^{\dagger}$. Berger extended this categorical equivalence to the equivalence between the category of $B$-pairs of $G_{K}$ with that of $\left(\varphi, \Gamma_{K}\right)$-modules over $\mathbf{B}_{\text {rig, },}^{\dagger}$, which we recall below.

We first note that we have a $\left(\varphi, G_{K}\right)$-equivariant canonical injection $\mathbf{B}_{\text {rig, }, K}^{\dagger} \hookrightarrow \widetilde{\mathbf{B}}_{\text {rig }}^{\dagger}$. Let $D$ be a $\left(\varphi, \Gamma_{K}\right)$-module over $\mathbf{B}_{\text {rig, }, K}^{\dagger}$ of rank $d$. For each $n \geqq n(D)$, we define

$$
W_{e}\left(D^{(n)}\right):=\left(\widetilde{\mathbf{B}}_{\text {rig }}^{\dagger, r_{n}}[1 / t] \otimes_{\mathbf{B}_{\text {rig }, K}^{\dagger, r_{n}}} D^{(n)}\right)^{\varphi=1} .
$$

Since we have an isomorphism

$$
\mathbf{B}_{\mathrm{rig}, K}^{\dagger, r_{n+1}} \otimes_{\varphi, \mathbf{B}_{\mathrm{rig}, K}^{\dagger, r_{n}}} D^{(n)} \stackrel{\sim}{\rightarrow} D^{(n+1)}: a \otimes x \mapsto a \varphi(x)
$$


and the map $\varphi: \widetilde{\mathbf{B}}_{\text {rig }}^{\dagger, r_{n}} \stackrel{\sim}{\rightarrow} \widetilde{\mathbf{B}}_{\text {rig }}^{\dagger, r_{n+1}}$ is isomorphism, we obtain a natural isomorphism

$$
\begin{aligned}
\widetilde{\mathbf{B}}_{\mathrm{rig}}^{\dagger, r_{n}} & \otimes_{\mathbf{B}_{\mathrm{rig}, K}^{\dagger, r_{n}}} D^{(n)} \stackrel{a \otimes x \mapsto \varphi(a) \otimes x}{\longrightarrow} \widetilde{\mathbf{B}}_{\mathrm{rig}}^{\dagger, r_{n+1}} \otimes_{\varphi, \mathbf{B}_{\mathrm{rig}, K}^{\dagger, r_{n}}} D^{(n)} \\
& \stackrel{\sim}{\rightarrow} \widetilde{\mathbf{B}}_{\mathrm{rig}}^{\dagger, r_{n+1}} \otimes_{\mathbf{B}_{\mathrm{rig}, K}^{\dagger, r_{n+1}}}\left(\mathbf{B}_{\mathrm{rig}, K}^{\dagger, r_{n+1}} \otimes_{\varphi, \mathbf{B}_{\mathrm{rig}, K}^{\dagger, r_{n}}} D^{(n)}\right) \stackrel{a \otimes(b \otimes x) \mapsto a \otimes b \varphi(x)}{\longrightarrow} \widetilde{\mathbf{B}}_{\mathrm{rig}}^{\dagger, r_{n+1}} \otimes_{\mathbf{B}_{\mathrm{rig}, K}^{\dagger, r_{n+1}}} D^{(n+1)},
\end{aligned}
$$

i.e., the map

$$
\varphi: \widetilde{\mathbf{B}}_{\mathrm{rig}}^{\dagger, r_{n}} \otimes_{\mathbf{B}_{\mathrm{rig}, K}^{\dagger, r_{n}}} D^{(n)} \rightarrow \widetilde{\mathbf{B}}_{\mathrm{rig}}^{\dagger, r_{n+1}} \otimes_{\mathbf{B}_{\mathrm{rig}, K}^{\dagger, r_{n+1}}} D^{(n+1)}: a \otimes x \mapsto \varphi(a) \otimes \varphi(x)
$$

is an isomorphism. Hence, we obtain the following diagram:

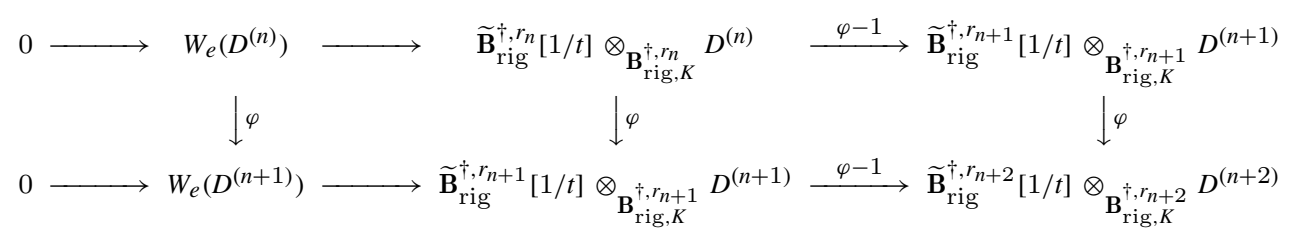

with exact rows. Hence, the map

$$
\varphi: W_{e}\left(D^{(n)}\right) \stackrel{\sim}{\rightarrow} W_{e}\left(D^{(n+1)}\right)
$$

is also an isomorphism. We define

$$
W_{e}(D):=W_{e}\left(D^{(n)}\right)
$$

for any $n \geqq n(D)$. Using the isomorphism $\varphi: W_{e}\left(D^{(n)}\right) \stackrel{\sim}{\rightarrow} W_{e}\left(D^{(n+1)}\right), W_{e}(D)$ does not depend on the choice of $n$. One has that $W_{e}(D)$ is a finite free $\mathbf{B}_{e}$-module of rank $d$, and the natural map

$$
\widetilde{\mathbf{B}}_{\text {rig }}^{\dagger, r_{n}}[1 / t] \otimes_{\mathbf{B}_{e}} W_{e}\left(D^{(n)}\right) \rightarrow \widetilde{\mathbf{B}}_{\text {rig }}^{\dagger, r_{n}}[1 / t] \otimes_{\mathbf{B}_{\text {rig }, K}^{\dagger, r_{n}}} D^{(n)}: a \otimes x \mapsto a x
$$

is an isomorphism by Proposition 2.2.6 of [5]. Put

$$
W_{\mathrm{dR}}(D):=\mathbf{B}_{\mathrm{dR}} \otimes_{\mathbf{B}_{e}} W_{e}(D) .
$$

Using the isomorphism above, we obtain an isomorphism

$$
\begin{gathered}
W_{\mathrm{dR}}(D) \stackrel{\sim}{\rightarrow} \mathbf{B}_{\mathrm{dR}} \otimes_{\mathbf{B}_{e}} W_{e}\left(D^{(n)}\right) \stackrel{\sim}{\rightarrow} \mathbf{B}_{\mathrm{dR}} \otimes_{\iota_{n}, \widetilde{\mathbf{B}}_{\mathrm{rig}}^{\dagger, r_{n}}[1 / t]}\left(\widetilde{\mathbf{B}}_{\mathrm{rig}}^{\dagger, r_{n}}[1 / t] \otimes_{\mathbf{B}_{e}} W_{e}\left(D^{(n)}\right)\right) \\
\stackrel{\sim}{\rightarrow} \mathbf{B}_{\mathrm{dR}} \otimes_{\iota_{n}, \widetilde{\mathbf{B}}_{\mathrm{rig}}^{\dagger, r_{n}}[1 / t]}\left(\widetilde{\mathbf{B}}_{\mathrm{rig}}^{\dagger, r_{n}}[1 / t] \otimes_{\mathbf{B}_{\mathrm{rig}, K}^{\dagger, r_{n}}} D^{(n)}\right) \stackrel{\sim}{\rightarrow} \mathbf{B}_{\mathrm{dR}} \otimes_{\iota_{n}, \mathbf{B}_{\mathrm{rig}, K}^{\dagger, r_{n}}} D^{(n)} .
\end{gathered}
$$

We define a $\mathbf{B}_{\mathrm{dR}}^{+}$-submodule

$$
W_{\mathrm{dR}}^{+}(D):=\mathbf{B}_{\mathrm{dR}}^{+} \otimes_{\iota_{n}, \mathbf{B}_{\mathrm{rig}, K}^{\dagger, r_{n}}} D^{(n)}
$$

of $W_{\mathrm{dR}}(D)$. Using the isomorphism

$$
\mathbf{B}_{\mathrm{dR}}^{+} \otimes_{\iota_{n}, \mathbf{B}_{\mathrm{rig}, K}^{\dagger, r_{n}}} D^{(n)} \stackrel{\sim}{\rightarrow} \mathbf{B}_{\mathrm{dR}}^{+} \otimes_{\iota_{n+1}, \mathbf{B}_{\mathrm{rig}, K}^{\dagger, r_{n+1}}} D^{(n+1)}: a \otimes x \mapsto a \otimes \varphi(x),
$$

$W_{\mathrm{dR}}^{+}(D)$ also does not depend on the choice of $n$. Hence, we obtain a $B$-pair $W(D):=\left(W_{e}(D), W_{\mathrm{dR}}^{+}(D)\right)$. 
The main theorem of $[5]$ is the following.

Theorem 2.19. The functor $D \mapsto W(D)$ is exact and gives an equivalence of categories between the category of $\left(\varphi, \Gamma_{K}\right)$-modules over $\mathbf{B}_{\text {rig, },}^{\dagger}$ and the category of B-pairs of $G_{K}$. Moreover, if we restrict this functor to étale $\left(\varphi, \Gamma_{K}\right)$-modules, this gives an equivalence of categories between the category of étale $\left(\varphi, \Gamma_{K}\right)$-modules over $\mathbf{B}_{\text {rig, },}^{\dagger}$ and the category of p-adic representations of $G_{K}$.

Proof. This is Theorem 2.2.7 and Proposition 2.2.9 of [5].

Remark 2.20. The inverse functor $D(-)$ of $W(-)$ is defined as follows; see $\S 2$ of [5] for the proof. Let $W=\left(W_{e}, W_{\mathrm{dR}}^{+}\right)$be a $B$-pair of $G_{K}$ of rank $d$. For each $n \geqq 1$, we first define

$$
\widetilde{D}^{(n)}(W):=\left\{x \in \widetilde{\mathbf{B}}_{\mathrm{rig}}^{\dagger, r_{n}}[1 / t] \otimes_{\mathbf{B}_{e}} W_{e} \mid \iota_{m}(x) \in W_{\mathrm{dR}}^{+} \text {for any } m \geqq n\right\} .
$$

Berger showed that $\widetilde{D}(W):=\lim _{\longrightarrow} \widetilde{D}^{(n)}(W)$ is a finite free $\widetilde{\mathbf{B}}_{\text {rig }}^{\dagger}$-module of rank $d$ with a $\left(\varphi, G_{K}\right)$-action. Then, $D(W)$ is defined as the unique $\left(\varphi, \Gamma_{K}\right)$-submodule $D(W) \subseteq$ $\widetilde{D}(W)^{\operatorname{Ker}(\chi)}$ over $\mathbf{B}_{\text {rig, }, K}^{\dagger}$ such that $\widetilde{\mathbf{B}}_{\text {rig }}^{\dagger} \otimes_{\mathbf{B}_{\text {rig }, K}^{\dagger}} D(W) \stackrel{\sim}{\rightarrow} \widetilde{D}(W)$.

Next, we recall the definition of Galois cohomology of $B$-pairs; see $\S 2$ of [25] and the appendix of [26] for details. For a continuous $G_{K}$-module $M$, and for each $q \geqq 0$, we denote by

$$
C^{q}\left(G_{K}, M\right):=\left\{c: G_{K}^{\times q} \rightarrow M \text { continuous map }\right\}
$$

the set of $q$ continuous cochains (when $q=0$, we define $G_{K}^{\times 0}:=\{$ one point $\}$ ). As usual, we define the map

$$
\delta_{q}: C^{q}\left(G_{K}, M\right) \rightarrow C^{q+1}\left(G_{K}, M\right)
$$

by

$$
\begin{aligned}
\delta_{q}(c)\left(g_{1}, g_{2}, \ldots, g_{q+1}\right):= & g_{1} c\left(g_{2}, \ldots, g_{q+1}\right)+(-1)^{q+1} c\left(g_{1}, g_{2}, \ldots, g_{q}\right) \\
& +\sum_{s=1}^{q}(-1)^{s} c\left(g_{1}, \ldots, g_{s-1}, g_{s} g_{s+1}, g_{s+2}, \ldots, g_{q+1}\right)
\end{aligned}
$$

and define the continuous cochain complex concentrated in degree $[0,+\infty)$ by

$$
C^{\bullet}\left(G_{K}, M\right):=\left[C^{0}\left(G_{K}, M\right) \stackrel{\delta_{0}}{\rightarrow} C^{1}\left(G_{K}, M\right) \stackrel{\delta_{1}}{\rightarrow} \cdots\right]
$$

We define

$$
\mathrm{H}^{q}(K, M):=\mathrm{H}^{q}\left(C^{\bullet}\left(G_{K}, M\right)\right) .
$$

For a $B$-pair $W:=\left(W_{e}, W_{\mathrm{dR}}^{+}\right)$, we denote by

$$
C^{\bullet}\left(G_{K}, W\right):=\operatorname{Cone}\left(C^{\bullet}\left(G_{K}, W_{e}\right) \oplus C^{\bullet}\left(G_{K}, W_{\mathrm{dR}}^{+}\right) \stackrel{\left(c_{e}, c_{\mathrm{dR}}\right) \mapsto c_{e}-c_{\mathrm{dR}}}{\longrightarrow} C^{\bullet}\left(G_{K}, W_{\mathrm{dR}}\right)\right)[-1]
$$

the degree (-1)-shift of the mapping cone of the map of complexes

$$
C^{\bullet}\left(G_{K}, W_{e}\right) \oplus C^{\bullet}\left(G_{K}, W_{\mathrm{dR}}^{+}\right) \rightarrow C^{\bullet}\left(G_{K}, W_{\mathrm{dR}}\right):\left(c_{e}, c_{\mathrm{dR}}\right) \mapsto c_{e}-c_{\mathrm{dR}}
$$


We define

$$
\mathrm{H}^{q}(K, W):=\mathrm{H}^{q}\left(C^{\bullet}\left(G_{K}, W\right)\right) .
$$

By the definition of mapping cone, we have the following long exact sequence:

$$
\begin{aligned}
& 0 \rightarrow \mathrm{H}^{0}(K, W) \rightarrow \mathrm{H}^{0}\left(K, W_{e}\right) \oplus \mathrm{H}^{0}\left(K, W_{\mathrm{dR}}^{+}\right) \rightarrow \mathrm{H}^{0}\left(K, W_{\mathrm{dR}}\right) \\
& \stackrel{\delta_{1, W}}{\longrightarrow} \mathrm{H}^{1}(K, W) \rightarrow \mathrm{H}^{1}\left(K, W_{e}\right) \oplus \mathrm{H}^{1}\left(K, W_{\mathrm{dR}}^{+}\right) \rightarrow \mathrm{H}^{1}\left(K, W_{\mathrm{dR}}\right) \\
& \stackrel{\delta_{2, W}}{\longrightarrow} \mathrm{H}^{2}(K, W) \rightarrow \mathrm{H}^{2}\left(K, W_{e}\right) \rightarrow 0,
\end{aligned}
$$

where the vanishings of $\mathrm{H}^{q}\left(K, W_{\mathrm{dR}}^{+}\right), \mathrm{H}^{q}\left(K, W_{\mathrm{dR}}\right), \mathrm{H}^{q+1}(K, W)$ and $\mathrm{H}^{q+1}\left(K, W_{e}\right)$ for any $q \geqq 2$ are proved in [26].

We define

$$
\mathbf{D}_{\mathrm{dR}}^{K}(W):=\mathrm{H}^{0}\left(K, W_{\mathrm{dR}}\right),
$$

and we define

$$
\exp _{K, W}:=\delta_{1, W}: \mathbf{D}_{\mathrm{dR}}^{K}(W) \rightarrow \mathrm{H}^{1}(K, W)
$$

as the first boundary map of the above exact sequence.

When $W=W(V)$, since we have a short exact sequence

$$
0 \rightarrow V \rightarrow \mathbf{B}_{e} \otimes_{\mathbb{Q}_{p}} V \oplus \mathbf{B}_{\mathrm{dR}}^{+} \otimes_{\mathbb{Q}_{p}} V \rightarrow \mathbf{B}_{\mathrm{dR}} \otimes_{\mathbb{Q}_{p}} V \rightarrow 0
$$

by Bloch and Kato, we have a canonical quasi-isomorphism

$$
C^{\bullet}\left(G_{K}, V\right) \rightarrow C^{\bullet}\left(G_{K}, W(V)\right) .
$$

This quasi-isomorphism gives an isomorphism

$$
\mathrm{H}^{q}(K, V) \stackrel{\sim}{\rightarrow} \mathrm{H}^{q}(K, W(V))
$$

for each $q$. By this isomorphism, the above exact sequence for $W=W(V)$ is equal to the exact sequence

$$
\begin{aligned}
& 0 \rightarrow \mathrm{H}^{0}(K, V) \rightarrow \mathrm{H}^{0}\left(K, \mathbf{B}_{e} \otimes_{\mathbb{Q}_{p}} V\right) \oplus \mathrm{H}^{0}\left(K, \mathbf{B}_{\mathrm{dR}}^{+} \otimes_{\mathbb{Q}_{p}} V\right) \rightarrow \mathrm{H}^{0}\left(K, \mathbf{B}_{\mathrm{dR}} \otimes_{\mathbb{Q}_{p}} V\right) \\
& \stackrel{\delta_{1, V}}{\longrightarrow} \mathrm{H}^{1}(K, V) \rightarrow \mathrm{H}^{1}\left(K, \mathbf{B}_{e} \otimes_{\mathbb{Q}_{p}} V\right) \oplus \mathrm{H}^{1}\left(K, \mathbf{B}_{\mathrm{dR}}^{+} \otimes_{\mathbb{Q}_{p}} V\right) \rightarrow \mathrm{H}^{1}\left(K, \mathbf{B}_{\mathrm{dR}} \otimes_{\mathbb{Q}_{p}} V\right) \\
& \stackrel{\delta_{2, V}}{\longrightarrow} \mathrm{H}^{2}(K, V) \rightarrow \mathrm{H}^{2}\left(K, \mathbf{B}_{e} \otimes_{\mathbb{Q}_{p}} V\right) \rightarrow 0,
\end{aligned}
$$

obtained from the Bloch-Kato fundamental short exact sequence.

The main result of this subsection is the following.

Theorem 2.21. Let $D$ be a $\left(\varphi, \Gamma_{K}\right)$-module over $\mathbf{B}_{\text {rig, } K}^{\dagger}$. For each $q \geqq 0$, there exist the following functorial isomorphisms.

(1) $\mathrm{H}^{q}(K, D) \stackrel{\sim}{\rightarrow} \mathrm{H}^{q}(K, W(D))$,

(2) $\mathrm{H}^{q}(K, D[1 / t]) \stackrel{\sim}{\rightarrow} \mathrm{H}^{q}\left(K, W_{e}(D)\right)$,

(3) $\mathrm{H}^{q}\left(K, \mathbf{D}_{\mathrm{dif}}^{+}(D)\right) \stackrel{\sim}{\rightarrow} \mathrm{H}^{q}\left(K, W_{\mathrm{dR}}^{+}(D)\right)$,

(4) $\mathrm{H}^{q}\left(K, \mathbf{D}_{\text {dif }}(D)\right) \stackrel{\sim}{\rightarrow} \mathrm{H}^{q}\left(K, W_{\mathrm{dR}}(D)\right)$. 
Moreover, these isomorphisms give an isomorphism between the exact sequence associated to $D$ in Theorem 2.8 and that associated to $W(D)$ defined above.

Proof. We proved (1) in Theorem 5.11 of [26]. Since we have $W_{\mathrm{dR}}^{+}(D)=$ $\mathbf{B}_{\mathrm{dR}}^{+} \otimes_{K_{\infty}[[t]]} \mathbf{D}_{\text {dif }}^{+}(D)$, then (3) follows Theorem 2.14 of [15]. (4) follows from (3) since we have $W_{\mathrm{dR}}(D)=\lim _{n \geqq 0} \frac{1}{t^{n}} W_{\mathrm{dR}}^{+}(D)$ and $\mathbf{D}_{\mathrm{dif}}(D)=\lim _{n \geqq 0} \frac{1}{t^{n}} \mathbf{D}_{\text {dif }}^{+}(D)$.

We prove (2) using (1). By (1), we have

$$
\mathrm{H}^{q}(K, D[1 / t]) \stackrel{\sim}{\rightarrow} \underset{n}{\lim } \mathrm{H}^{q}\left(K, \frac{1}{t^{n}} D\right) \stackrel{\sim}{\rightarrow} \underset{n}{\lim } \mathrm{H}^{q}\left(K, W\left(\frac{1}{t^{n}} D\right)\right) .
$$

Since we have $W\left(\frac{1}{t^{n}} D\right) \stackrel{\sim}{\rightarrow}\left(W_{e}(D), \frac{1}{t^{n}} W_{\mathrm{dR}}^{+}(D)\right)$ for each $n \geqq 0$, we obtain

$$
\underset{n \geqq 0}{\lim _{n \rightarrow 0}} C^{\bullet}\left(G_{K}, W_{\mathrm{dR}}^{+}\left(\frac{1}{t^{n}} D\right)\right)=\underset{n \geqq 0}{\lim } C^{\bullet}\left(G_{K}, \frac{1}{t^{n}} W_{\mathrm{dR}}^{+}(D)\right)=C^{\bullet}\left(G_{K}, W_{\mathrm{dR}}(D)\right) .
$$

Hence, we obtain an isomorphism

$$
\begin{aligned}
\lim _{n \geqq 0} & \mathrm{H}^{q}\left(K, W\left(\frac{1}{t^{n}} D\right)\right) \stackrel{\sim}{\rightarrow} \mathrm{H}^{q}\left(\underset{n \geqq 0}{\underset{n}{\longrightarrow}} \mathrm{C}^{\bullet}\left(G_{K}, W\left(\frac{1}{t^{n}}(D)\right)\right)\right) \\
& \stackrel{\sim}{\rightarrow} \mathrm{H}^{q}\left(\operatorname { C o n e } \left(C^{\bullet}\left(G_{K}, W_{e}(D)\right) \oplus C^{\bullet}\left(G_{K}, W_{\mathrm{dR}}(D)\right)\right.\right. \\
& \left.\left.\stackrel{\left(c_{e}, c_{\mathrm{dR}}\right) \mapsto c_{e}-c_{\mathrm{dR}}}{\longrightarrow} C^{\bullet}\left(G_{K}, W_{\mathrm{dR}}(D)\right)\right)[-1]\right) .
\end{aligned}
$$

Since we have the following short exact sequence of complexes:

$$
\begin{aligned}
0 & \rightarrow C^{\bullet}\left(G_{K}, W_{e}(D)\right) \stackrel{x \mapsto(x, x)}{\longrightarrow} C^{\bullet}\left(G_{K}, W_{e}(D)\right) \oplus C^{\bullet}\left(G_{K}, W_{\mathrm{dR}}(D)\right) \\
& \stackrel{(x, y) \mapsto x-y}{\longrightarrow} C^{\bullet}\left(G_{K}, W_{\mathrm{dR}}(D)\right) \rightarrow 0,
\end{aligned}
$$

we obtain a natural isomorphism

$$
\begin{aligned}
& \mathrm{H}^{q}\left(K, W_{e}(D)\right) \\
& \quad \stackrel{\sim}{\rightarrow} \mathrm{H}^{q}\left(\operatorname { C o n e } \left(C^{\bullet}\left(G_{K}, W_{e}(D)\right) \oplus C^{\bullet}\left(G_{K}, W_{\mathrm{dR}}(D)\right)\right.\right. \\
& \left.\left.\quad \stackrel{\left(c_{e}, c_{\mathrm{dR}}\right) \mapsto c_{e}-c_{\mathrm{dR}}}{\longrightarrow} C^{\bullet}\left(G_{K}, W_{\mathrm{dR}}(D)\right)\right)[-1]\right),
\end{aligned}
$$

which proves (2).

Next, we prove that the isomorphisms (1)-(4) of the theorem give an isomorphism of the corresponding long exact sequences. Since the other commutativities are easy to check, it suffices to show that the following two diagrams are commutative

(i)

$$
\begin{array}{rlr}
\mathrm{H}^{0}\left(K, \mathbf{D}_{\mathrm{dif}}(D)\right) & \stackrel{\sim}{\rightarrow} & \mathrm{H}^{0}\left(K, W_{\mathrm{dR}}(D)\right) \\
\downarrow \exp _{K, D} & & \\
& \stackrel{\sim}{\rightarrow} & \exp _{K, W(D)} \\
\mathrm{H}^{1}(K, D) & \stackrel{\longrightarrow}{\longrightarrow} & \mathrm{H}^{1}(K, W(D))
\end{array}
$$


(ii)

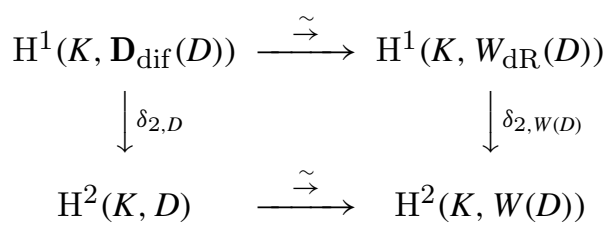

We first prove the commutativity of (i). For simplicity, we assume that $\Gamma_{K}$ has a topological generator $\gamma_{K}$; the general case can be proved similarly using the argument of $\S 2.1$ of [23]. If we denote by $\operatorname{Ext}^{1}\left(\mathbf{B}_{\mathrm{rig}, K}^{\dagger}, D\right)$ (respectively $\left.\operatorname{Ext}^{1}\left(\left(\mathbf{B}_{e}, \mathbf{B}_{\mathrm{dR}}^{+}\right), W(D)\right)\right)$ the group of extension classes of $\mathbf{B}_{\text {rig, } K}^{\dagger}$ by $D$ (respectively of the trivial $B$-pair $\left(\mathbf{B}_{e}, \mathbf{B}_{\mathrm{dR}}^{+}\right.$) by $W(D))$, then we have the following canonical isomorphisms

$$
h_{D}: \mathrm{H}^{1}(K, D) \stackrel{\sim}{\rightarrow} \operatorname{Ext}^{1}\left(\mathbf{B}_{\mathrm{rig}, K}^{\dagger}, D\right), \quad h_{W(D)}: \mathrm{H}^{1}(K, W(D)) \stackrel{\sim}{\rightarrow} \operatorname{Ext}^{1}\left(\left(\mathbf{B}_{e}, \mathbf{B}_{\mathrm{dR}}^{+}\right), W(D)\right)
$$

by $\S 2.1$ of [23] and by $\S 2.1$ of [25], and we have the following commutative diagram

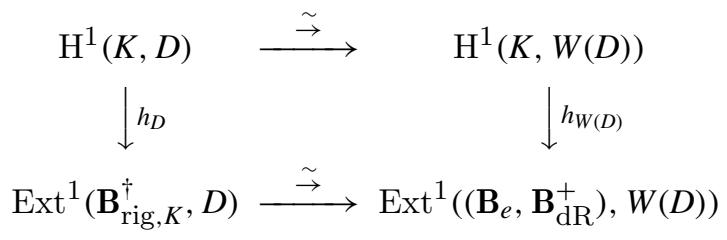

by Theorem 5.11 of [26], where the isomorphism

$$
\operatorname{Ext}^{1}\left(\mathbf{B}_{\mathrm{rig}, K}^{\dagger}, D\right) \stackrel{\sim}{\rightarrow} \operatorname{Ext}^{1}\left(\left(\mathbf{B}_{e}, \mathbf{B}_{\mathrm{dR}}^{+}\right), W(D)\right)
$$

is given by

$$
\left[0 \rightarrow \mathbf{B}_{\mathrm{rig}, K}^{\dagger} \rightarrow D^{\prime} \rightarrow D \rightarrow 0\right] \mapsto\left[0 \rightarrow\left(\mathbf{B}_{e}, \mathbf{B}_{\mathrm{dR}}^{+}\right) \rightarrow W\left(D^{\prime}\right) \rightarrow W(D) \rightarrow 0\right],
$$

i.e., given by applying the functor $W(-)$.

Let $a \in \mathrm{H}^{0}\left(K, \mathbf{D}_{\text {dif }}(D)\right)=\mathbf{D}_{\text {dif }}(D)^{\gamma_{K}=1} \stackrel{\sim}{\rightarrow} \mathrm{H}^{0}\left(K, W_{\mathrm{dR}}(D)\right)$. By the above diagram, it suffices to show that the functor $W(-)$ sends the extension corresponding to $\exp _{K, D}(a)$ to the extension corresponding to $\exp _{K, W(D)}(a)$.

Take $n$ sufficiently large such that $a \in \mathbf{D}_{\text {dif, } n}(D)^{\gamma_{K}=1}$. By (1) of Lemma 2.12, and by the definition of the isomorphism $\mathrm{H}^{1}(K, D) \stackrel{\sim}{\rightarrow} \operatorname{Ext}^{1}\left(\mathbf{B}_{\text {rig, },}^{\dagger}, D\right)$, if we take $\tilde{a} \in D^{(n)}[1 / t]$ such that $\iota_{m}(\tilde{a})-a \in \mathbf{D}_{\text {dif, } m}^{+}(D)$ for any $m \geqq n$, the extension $D_{a}$ corresponding to $\exp _{K, D}(a)$ is explicitly defined by

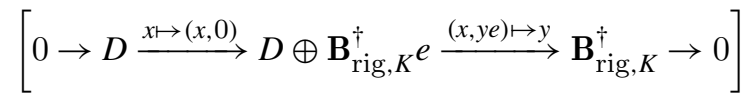

such that

$$
\begin{aligned}
\varphi((x, y e)) & :=\left(\varphi(x)+\varphi(y)\left(\gamma_{K}-1\right) \tilde{a}, \varphi(y) e\right), \\
\gamma_{K}((x, y e)) & :=\left(\gamma_{K}(x)+\gamma_{K}(y)(\varphi-1) \tilde{a}, \gamma_{K}(y) e\right)
\end{aligned}
$$

for any $(x, y e) \in D \oplus \mathbf{B}_{\text {rig, } K^{\dagger}}^{e}$. 
On the other hand, the extension

$$
W_{a}:=\left(W_{e, a}:=W_{e}(D) \oplus \mathbf{B}_{e} e_{\mathrm{crys}}, W_{\mathrm{dR}, a}^{+}:=W_{\mathrm{dR}}^{+}(D) \oplus \mathbf{B}_{\mathrm{dR}}^{+} e_{\mathrm{dR}}\right)
$$

corresponding to $\exp _{K, W(D)}(a)$ is defined by

$$
g\left(x+y e_{\mathrm{crys}}\right):=g(x)+g(y) e_{\mathrm{crys}}, \quad g\left(x^{\prime}+y^{\prime} e_{\mathrm{dR}}\right):=g\left(x^{\prime}\right)+g\left(y^{\prime}\right) e_{\mathrm{dR}}
$$

for any $g \in G_{K}, x \in W_{e}(D), x^{\prime} \in W_{\mathrm{dR}}^{+}(D), y \in \mathbf{B}_{e}, y^{\prime} \in \mathbf{B}_{\mathrm{dR}}^{+}$, and the inclusion

$$
W_{\mathrm{dR}, a}^{+} \hookrightarrow \mathbf{B}_{\mathrm{dR}} \otimes_{\mathbf{B}_{e}} W_{e, a}=W_{\mathrm{dR}}(D) \oplus \mathbf{B}_{\mathrm{dR}} e_{\mathrm{crys}}
$$

is defined by

$$
x+y e_{\mathrm{dR}} \mapsto(x+y a)+y e_{\mathrm{crys}}\left(x \in W_{\mathrm{dR}}^{+}(D), y \in \mathbf{B}_{\mathrm{dR}}^{+}\right) .
$$

Let us show that $D\left(W_{a}\right) \stackrel{\sim}{\rightarrow} D_{a}$ as an extension. By the definition of $\tilde{a}$, we can easily check that $\widetilde{D}^{(n)}\left(W_{a}\right)$ defined in Remark 2.20 is isomorphic to

$$
\widetilde{\mathbf{B}}_{\text {rig }}^{\dagger, r_{n}} \otimes_{\mathbf{B}_{\text {rig }, K}^{\dagger, r_{n}}} D^{(n)} \oplus \widetilde{\mathbf{B}}_{\text {rig }}^{\dagger, r_{n}}\left(e_{\text {crys }}+\tilde{a}\right) \subseteq \widetilde{\mathbf{B}}_{\text {rig }}^{\dagger, r_{n}}[1 / t] \otimes_{\mathbf{B}_{e}} W_{e, a}
$$

and that $\widetilde{D}\left(W_{a}\right)$ contains a $\left(\varphi, \Gamma_{K}\right)$-module $D \oplus \mathbf{B}_{\text {rig, } K}^{\dagger}\left(e_{\text {crys }}+\tilde{a}\right)$ over $\mathbf{B}_{\text {rig, },}^{\dagger}$, which is easily seen to be isomorphic to $D_{a}$, hence finishing the proof of the commutativity of (i).

Finally, we prove the commutativity of (ii). By Lemma 2.15 (we note that we can show the $B$-pairs analogue of Lemma 2.15 in the same way), it suffices to show that the following diagram is commutative:

(ii) ${ }^{\prime}$

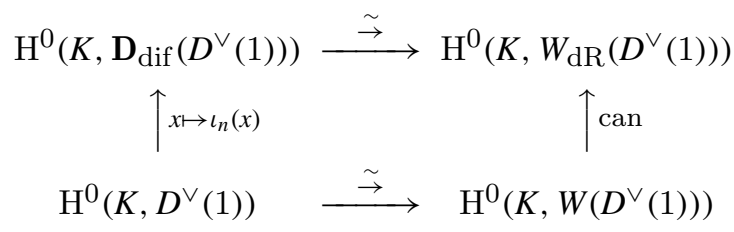

The commutativity of this diagram is trivial. We finish the proof of the theorem.

\section{The Perrin-Riou big exponential map for de Rham $(\varphi, \Gamma)$-modules}

This section is the main part of this article. For any de Rham $(\varphi, \Gamma)$-module $D$, we construct a system of maps $\left\{\operatorname{Exp}_{D, h}\right\}_{h \gg 0}$, which we call big exponential maps, and prove their important properties, i.e., their interpolation formulae and theorem $\delta(D)$. The first two subsections deal with preliminaries. In $\S 3.1$, we recall Pottharst's theory of the analytic Iwasawa cohomologies [32]. In $\S 3.2$, we recall Berger's construction of $p$-adic differential equations associated to de Rham $(\varphi, \Gamma)$-modules $[3,6]$. The next two subsections form the main part of this article. In $\S 3.3$, we define the maps $\left\{\operatorname{Exp}_{D, h}\right\}_{h \gg 0}$ and prove their interpolation formulae. In $\S 3.4$, we formulate and prove theorem $\delta(D)$. In $\S 3.5$, we compare our big exponential maps and our theorem $\delta(D)$ with those of Perrin-Riou or Pottharst in the crystalline case. 


\subsection{Analytic Iwasawa cohomology}

In this subsection, we recall the results of Pottharst concerning analytic Iwasawa cohomologies of $(\varphi, \Gamma)$-modules over the Robba ring [32].

Let $\Lambda:=\mathbb{Z}_{p}\left[\left[\Gamma_{K}\right]\right]:=\lim _{n} \mathbb{Z}_{p}\left[\Gamma_{K} / \Gamma_{K_{n}}\right]$ be the Iwasawa algebra of $\Gamma_{K}$. If we decompose $\Gamma_{K}$ by $\Gamma_{K} \stackrel{\sim}{\rightarrow} \Gamma_{K}$,tor $\times \Gamma_{K}$,free, where $\Gamma_{K}$,tor $\subseteq \Gamma_{K}$ is the torsion subgroup of $\Gamma_{K}$ and $\Gamma_{K, \text { free }}=\Gamma_{K} \cap \chi^{-1}\left(1+2 p \mathbb{Z}_{p}\right)$, then we have an isomorphism $\Lambda \stackrel{\sim}{\rightarrow} \mathbb{Z}_{p}\left[\Gamma_{K, \text { tor }}\right] \otimes_{\mathbb{Z}_{p}} \mathbb{Z}_{p}$

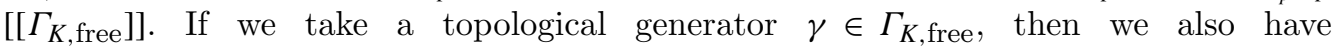
a $\mathbb{Z}_{p}\left[\Gamma_{K, \text { tor }}\right]$-algebra isomorphism $\mathbb{Z}_{p}\left[\Gamma_{K, \text { tor }}\right] \otimes_{\mathbb{Z}_{p}} \mathbb{Z}_{p}\left[\left[\Gamma_{K, \text { free }}\right] \stackrel{\sim}{\rightarrow} \mathbb{Z}_{p}\left[\Gamma_{K, \text { tor }}\right] \otimes_{\mathbb{Z}_{p}} \mathbb{Z}_{p}[[T]]\right.$ defined by $1 \otimes[\gamma] \mapsto 1 \otimes(1+T)$.

Let $\mathfrak{m} \subseteq \Lambda$ be the Jacobson radical of $\Lambda$. For each $n \geqq 1$, we set $\Lambda_{n}:=\Lambda\left[\frac{\mathfrak{m}^{n}}{p}\right]^{\wedge}[1 / p]$, where $\Lambda\left[\frac{\mathfrak{m}^{n}}{p}\right]^{\wedge}$ is the $p$-adic completion of $\Lambda\left[\frac{\mathfrak{m}^{n}}{p}\right]$, which is an affinoid algebra over $\mathbb{Q}_{p}$. The natural map $\Lambda\left[\frac{\mathfrak{m}^{n+1}}{p}\right] \rightarrow \Lambda\left[\frac{\mathfrak{m}^{n}}{p}\right]$ induces a continuous map $\Lambda_{n+1} \rightarrow \Lambda_{n}$ for each $n \geqq 1$. We set $\Lambda_{\infty}:=\lim _{\longleftarrow} \Lambda_{n}$. If we fix an isomorphism $\Lambda \stackrel{\sim}{\rightarrow} \mathbb{Z}_{p}\left[\Gamma_{K, \text { tor }}\right] \otimes_{\mathbb{Z}_{p}} \mathbb{Z}_{p}[[T]]$ as above, then this isomorphism naturally extends to a $\mathbb{Q}_{p}\left[\Gamma_{K \text {,tor }}\right]$-algebra isomorphism $\Lambda_{\infty} \stackrel{\sim}{\rightarrow} \mathbb{Q}_{p}\left[\Gamma_{K, \text { tor }}\right] \otimes_{\mathbb{Q}_{p}} \mathbf{B}_{\text {rig, } \mathbb{Q}_{p}}^{+}$, where the ring $\mathbf{B}_{\text {rig, } \mathbb{Q}_{p}}^{+}$is defined by

$$
\mathbf{B}_{\text {rig, } \mathbb{Q}_{p}}^{+}:=\left\{f(T)=\sum_{n=0}^{\infty} a_{n} T^{n} \mid a_{n} \in \mathbb{Q}_{p} \text { and } f(T) \text { is convergent on } 0 \leqq|T|<1\right\} \text {. }
$$

We remark that the above isomorphism $\Lambda_{\infty} \stackrel{\sim}{\rightarrow} \mathbb{Q}_{p}\left[\Gamma_{K}\right.$, tor $] \otimes_{\mathbb{Q}_{p}} \mathbf{B}_{\text {rig, }, \mathbb{Q}_{p}}^{+}$also depends on the choice of a topological generator $\gamma$ and is highly non-canonical, and is only used to help the reader to understand the structure of $\Lambda_{\infty}$.

We define $\Lambda_{n}\left[\Gamma_{K}\right]$-modules $\widetilde{\Lambda}_{n}$ and $\widetilde{\Lambda}_{n}^{\iota}$ by $\widetilde{\Lambda}_{n}=\widetilde{\Lambda}_{n}^{\iota}=\Lambda_{n}$ as a $\Lambda_{n}$-module and $\gamma(\lambda):=[\gamma] \cdot \lambda, \gamma\left(\lambda^{\prime}\right):=\left[\gamma^{-1}\right] \cdot \lambda^{\prime}$ for $\lambda \in \tilde{\Lambda}_{n}, \lambda^{\prime} \in \tilde{\Lambda}_{n}^{\iota}$ and $\gamma \in \Gamma_{K}$.

Let $D$ be a $\left(\varphi, \Gamma_{K}\right)$-module over $\mathbf{B}_{\text {rig, } K}^{\dagger}$. For each $n \geqq 1$, we define a $\left(\varphi, \Gamma_{K}\right)$-module $D \widehat{\otimes}_{\mathbb{Q}_{p}} \widetilde{\Lambda}_{n}^{\iota}$ over $\mathbf{B}_{\text {rig, } K}^{\dagger} \widehat{\otimes}_{\mathbb{Q}_{p}} \Lambda_{n}$ as follows (see $\S 2$ of [32] for a more precise definition). We define $D \widehat{\otimes}_{\mathbb{Q}_{p}} \widetilde{\Lambda}_{n}^{l}:=D \widehat{\otimes}_{\mathbb{Q}_{p}} \Lambda_{n}$ as a $\mathbf{B}_{\text {rig, },}^{\dagger} \widehat{\otimes}_{\mathbb{Q}_{p}} \Lambda_{n}$-module and define $\varphi(x \widehat{\otimes} \lambda):=\varphi(x) \widehat{\otimes} \lambda$, $\psi(x \widehat{\otimes} \lambda):=\psi(x) \widehat{\otimes} \lambda$ and $\gamma(x \widehat{\otimes} \lambda):=\gamma(x) \widehat{\otimes}\left[\gamma^{-1}\right] \cdot \lambda$ for $x \in D, \lambda \in \Lambda_{n}$ and $\gamma \in \Gamma_{K}$.

For each $n \geqq 1$, we define two complexes $C_{\varphi, \gamma_{K}}^{\bullet}\left(D \widehat{\otimes}_{\mathbb{Q}_{p}} \widetilde{\Lambda}_{n}^{\iota}\right)$ and $C_{\psi, \gamma_{K}}^{\bullet}\left(D \widehat{\otimes}_{\mathbb{Q}_{p}} \widetilde{\Lambda}_{n}^{\iota}\right)$ and define the natural map of complexes $C_{\varphi, \gamma_{K}}^{\bullet}\left(D \widehat{\otimes}_{\mathbb{Q}_{p}} \widetilde{\Lambda}_{n}^{l}\right) \rightarrow C_{\psi, \gamma_{K}}^{\bullet}\left(D \widehat{\otimes}_{\mathbb{Q}_{p}} \widetilde{\Lambda}_{n}^{l}\right)$ in the same way as those for $D$. We define $\mathrm{H}^{q}\left(K, D \widehat{\otimes}_{\mathbb{Q}_{p}} \widetilde{\Lambda}_{n}^{\iota}\right):=\mathrm{H}^{q}\left(C_{\varphi, \gamma_{K}}^{\bullet}\left(D \widehat{\otimes}_{\mathbb{Q}_{p}} \widetilde{\Lambda}_{n}^{\iota}\right)\right)$, which is a $\Lambda_{n}$-module. The natural map $\Lambda_{n+1} \rightarrow \Lambda_{n}$ induces a natural map $D \widehat{\otimes}_{\mathbb{Q}_{p}} \widetilde{\Lambda}_{n+1}^{\iota} \rightarrow D \widehat{\otimes}_{\mathbb{Q}_{p}} \widetilde{\Lambda}_{n}^{\iota}$, and this map induces $\mathrm{H}^{q}\left(K, D \widehat{\otimes}_{\mathbb{Q}_{p}} \widetilde{\Lambda}_{n+1}^{\iota}\right) \rightarrow \mathrm{H}^{q}\left(K, D \widehat{\otimes}_{\mathbb{Q}_{p}} \widetilde{\Lambda}_{n}^{\iota}\right)$. Following [32], we define the analytic Iwasawa cohomology of $D$ as follows.

Definition 3.1. Let $D$ be a $\left(\varphi, \Gamma_{K}\right)$-module over $\mathbf{B}_{\mathrm{rig}, K}^{\dagger}$, and let $q \geqq 0$ be an integer. We define the $q$ th analytic Iwasawa cohomology of $D$ by

$$
\mathbf{H}_{\mathrm{Iw}}^{q}(K, D):={\underset{\lim }{n}}_{\mathrm{H}^{q}}^{q}\left(K, D \widehat{\otimes}_{\mathbb{Q}_{p}} \widetilde{\Lambda}_{n}^{\iota}\right),
$$

which is a $\Lambda_{\infty}$-module. 
Because we have a decomposition $\mathbb{Q}_{p}\left[\Gamma_{K}\right.$,tor $]=\bigoplus_{\eta \in \widehat{\Gamma}_{K, \text { tor }}} \mathbb{Q}_{p} \alpha_{\eta}$, where $\widehat{\Gamma}_{K \text {,tor }}$ is the character group of $\Gamma_{K}$,tor and $\alpha_{\eta}$ is the idempotent corresponding to $\eta$, we also have $\Lambda_{\infty}=\bigoplus_{\eta \in \hat{\Gamma}_{K, \text { tor }}} \Lambda_{\infty} \alpha_{\eta}$, and each $\Lambda_{\infty} \alpha_{\eta}$ is non-canonically isomorphic to $\mathbf{B}_{\text {rig, } \mathbb{Q}_{p}}^{+}$. Let $M$ be a $\Lambda_{\infty}$-module. Using this decomposition, we obtain a decomposition $M=\bigoplus_{\eta \in \widehat{\Gamma}_{K, \text { tor }}} M_{\eta}$, where we define $M_{\eta}:=\alpha_{\eta} M$ which is a $\Lambda_{\infty} \alpha_{\eta}$-module. For a $\mathbf{B}_{\text {rig, } \mathbb{Q}_{p}}^{+}$-module $N$, we define $N_{\text {tor }}:=\left\{x \in N \mid a x=0\right.$ for some non zero $\left.a \in \mathbf{B}_{\text {rig, } \mathbb{Q}_{p}}^{+}\right\}$. For a $\Lambda_{\infty}$-module $M$, we define $M_{\text {tor }}:=\bigoplus_{\eta \in \widehat{\Gamma}_{K, \text { tor }}}\left(M_{\eta}\right)_{\text {tor }}$.

As for the fundamental properties of $\mathbf{H}_{\mathrm{Iw}}^{q}(K, D)$, Pottharst proved the following results, which are a generalization of Perrin-Riou's results [29] in the case of $p$-adic Galois representations.

Theorem 3.2. Let $D$ be a $\left(\varphi, \Gamma_{K}\right)$-module over $\mathbf{B}_{\text {rig, }, K}^{\dagger}$ of rank $d$. Then we have the following.

(1) For each $n \geqq 1$ and $q \geqq 0$, the natural map

$$
\mathrm{H}^{q}\left(K, D \widehat{\otimes}_{\mathbb{Q}_{p}} \widetilde{\Lambda}_{n+1}^{\iota}\right) \rightarrow \mathrm{H}^{q}\left(K, D \widehat{\otimes}_{\mathbb{Q}_{p}} \widetilde{\Lambda}_{n}^{\iota}\right)
$$

induces an isomorphism of $\Lambda_{n}$-modules

$$
\mathrm{H}^{q}\left(K, D \widehat{\otimes}_{\mathbb{Q}_{p}} \widetilde{\Lambda}_{n+1}^{l}\right) \otimes_{\Lambda_{n+1}} \Lambda_{n} \stackrel{\sim}{\rightarrow} \mathrm{H}^{q}\left(K, D \widehat{\otimes}_{\mathbb{Q}_{p}} \widetilde{\Lambda}_{n}^{l}\right),
$$

(2) $\mathbf{H}_{\mathrm{Iw}}^{q}(K, D)=0$ if $q \neq 1,2$,

(3) $\mathbf{H}_{\mathrm{Iw}}^{1}(K, D)_{\text {tor }}$ and $\mathbf{H}_{\mathrm{Iw}}^{2}(K, D)$ are finite-dimensional $\mathbb{Q}_{p}$-vector spaces,

(4) $\mathbf{H}_{\mathrm{Iw}}^{1}(K, D) / \mathbf{H}_{\mathrm{Iw}}^{1}(K, D)_{\text {tor }}$ is a finite free $\Lambda_{\infty}$-module of rank $d\left[K: \mathbb{Q}_{p}\right]$.

Proof. This is Theorem 2.6 and Proposition 2.9 of [32].

Let $A$ be a $\mathbb{Q}_{p}$-affinoid algebra, and let $\delta: \Gamma_{K} \rightarrow A^{\times}$be a continuous homomorphism. We define $A(\delta):=A e_{\delta}$ the free rank-1 $A$-module with the base $e_{\delta}$ with an $A$-linear $\Gamma_{K}$ action by $\gamma\left(e_{\delta}\right):=\delta(\gamma) e_{\delta}$ for $\gamma \in \Gamma_{K}$. Then the continuous $\mathbb{Q}_{p}$-algebra homomorphism $f_{\delta}: \Lambda_{\infty} \rightarrow A$ which is defined by $f_{\delta}([\gamma]):=\delta(\gamma)^{-1}$ for any $\gamma \in \Gamma_{K}$ induces the isomorphism

$$
D \hat{\otimes} \mathbb{Q}_{p}\left(\widetilde{\Lambda}_{\infty}^{\iota} \otimes_{\Lambda_{\infty}, f_{\delta}} A\right) \stackrel{\sim}{\rightarrow} D \hat{\otimes} \mathbb{Q}_{p} A(\delta): x \hat{\otimes}(\lambda \otimes a) \mapsto x \hat{\otimes} f_{\delta}(\lambda) a e_{\delta}
$$

of $\left(\varphi, \Gamma_{K}\right)$-modules over $\mathbf{B}_{\text {rig, } K}^{\dagger} \hat{\otimes}_{\mathbb{Q}_{p}} A$. This isomorphism induces the canonical projection map

$$
\mathbf{H}_{\mathrm{Iw}}^{q}(K, D) \rightarrow \mathrm{H}^{q}\left(K, D \hat{\otimes} \mathbb{Q}_{p}\left(\widetilde{\Lambda}_{\infty}^{\iota} \otimes_{\Lambda_{\infty}, f_{\delta}} A\right)\right) \stackrel{\sim}{\rightarrow} \mathrm{H}^{q}\left(K, D \hat{\otimes} \mathbb{Q}_{p} A(\delta)\right) .
$$

For each $L=K_{n}(n \geqq 1)$ or $L=K, k \in \mathbb{Z}$ and $q \geqq 0$, as a special case of the above projection map, we define the canonical map

$$
\operatorname{pr}_{L, D(k)}: \mathbf{H}_{\mathrm{Iw}}^{q}(K, D) \rightarrow \mathrm{H}^{q}(L, D(k))
$$

as follows. First, define the continuous homomorphism $\delta_{L}: \Gamma_{K} \rightarrow \mathbb{Q}_{p}\left[\Gamma_{K} / \Gamma_{L}\right]^{\times}: \gamma \mapsto$ $[\bar{\gamma}]^{-1}$. Then, for each $k \in \mathbb{Z}$, we obtain the projection map

$$
\mathbf{H}_{\mathrm{Iw}}^{q}(K, D) \rightarrow \mathrm{H}^{q}\left(K, D \otimes_{\mathbb{Q}_{p}} \mathbb{Q}_{p}\left[\Gamma_{K} / \Gamma_{L}\right]\left(\delta_{L} \chi^{k}\right)\right)
$$


associated to the character $\delta_{L} \chi^{k}$. Using the isomorphism $D \otimes \mathbb{Q}_{p} \mathbb{Q}_{p}\left[\Gamma_{K} / \Gamma_{L}\right]\left(\delta_{L} \chi^{k}\right) \stackrel{\sim}{\rightarrow}$ $D(k) \otimes \mathbb{Q}_{p} \mathbb{Q}_{p}\left[\widetilde{\Gamma_{K} / \Gamma_{L}}\right]^{\imath}: x \otimes a e_{\delta_{L} \chi^{k}} \mapsto\left(x \otimes e_{k}\right) \otimes a$ (where $\mathbb{Q}_{p}\left[\widetilde{\Gamma_{K} / \Gamma_{L}}\right]^{\iota}$ is defined similarly as $\left.\widetilde{\Lambda}_{\infty}^{\iota}\right)$, we define

$$
\begin{aligned}
\operatorname{pr}_{L, D(k)}: \mathbf{H}_{\mathrm{Iw}}^{q}(K, D) & \rightarrow \mathrm{H}^{q}\left(K, D \otimes_{\mathbb{Q}_{p}} \mathbb{Q}_{p}\left[\Gamma_{K} / \Gamma_{L}\right]\left(\delta_{L} \chi^{k}\right)\right) \\
& \stackrel{\sim}{\rightarrow} \mathrm{H}^{q}\left(K, D(k) \otimes_{\mathbb{Q}_{p}} \mathbb{Q}_{p}\left[\widehat{\Gamma_{K} / \Gamma_{L}}\right]\right) \\
& \stackrel{\sim}{\rightarrow} \mathrm{H}^{q}(L, D(k)),
\end{aligned}
$$

where the last isomorphism is the canonical one induced by Shapiro's lemma (see Theorem 2.2 of [23]).

For each $k \in \mathbb{Z}$, we define a canonical isomorphism

$$
f_{D, k}: \mathbf{H}_{\mathrm{IW}}^{q}(K, D) \stackrel{\sim}{\rightarrow} \mathbf{H}_{\mathrm{IW}}^{q}(K, D(k))
$$

of $\mathbb{Q}_{p}$-vector spaces as follows. We first define continuous $\mathbb{Q}_{p}$-algebra isomorphisms $f_{k}: \Lambda_{0} \stackrel{\sim}{\rightarrow} \Lambda_{0}\left(\Lambda_{0}=\Lambda, \Lambda_{n}, \Lambda_{\infty}\right)$ by $f_{k}([\gamma]):=\chi(\gamma)^{-k}[\gamma]$ for any $\gamma \in \Gamma_{K}$. Using $f_{k}$, for each $n \geqq 1$, we define a continuous $\mathbf{B}_{\text {rig, } K^{-}}^{\dagger}$ linear isomorphism $D \widehat{\otimes}_{\mathbb{Q}_{p}} \widetilde{\Lambda}_{n}^{\iota} \stackrel{\sim}{\rightarrow} D(k) \widehat{\otimes}_{\mathbb{Q}_{p}} \widetilde{\Lambda}_{n}^{\iota}$ : $x \widehat{\otimes} \lambda \mapsto\left(x \otimes e_{k}\right) \widehat{\otimes} f_{k}(\lambda)$. This map commutes with $\varphi$ and $\Gamma_{K}$-actions, and hence induces an isomorphism $C_{\varphi, \gamma_{K}}^{\bullet}\left(D \widehat{\otimes}_{\mathbb{Q}_{p}} \widetilde{\Lambda}_{n}^{l}\right) \stackrel{\sim}{\rightarrow} C_{\varphi, \gamma_{K}}^{\bullet}\left(D(k) \widehat{\otimes}_{\mathbb{Q}_{p}} \widetilde{\Lambda}_{n}^{l}\right)$ of complexes of $\mathbb{Q}_{p}$-vector spaces, and hence also induces an isomorphism $f_{D, k}: \mathbf{H}_{\mathrm{Iw}}^{q}(K, D) \stackrel{\sim}{\rightarrow} \mathbf{H}_{\mathrm{Iw}}^{q}(K, D(k))$ of $\mathbb{Q}_{p}$-vector spaces for each $q$.

Using the $\psi$-complex $C_{\psi, \gamma_{K}}^{\bullet}\left(D \widehat{\otimes}_{\mathbb{Q}_{p}} \widetilde{\Lambda}_{n}^{\iota}\right)$, we can describe $\mathbf{H}_{\mathrm{Iw}}^{q}(K, D)$ in a more explicit way as follows.

Theorem 3.3. Let $D$ be a $\left(\varphi, \Gamma_{K}\right)$-module over $\mathbf{B}_{\mathrm{rig}, K}^{\dagger}$.

(1) For each $n \geqq 1$, the map

$$
\left(\gamma_{K}-1\right):\left(D \widehat{\otimes}_{\mathbb{Q}_{p}} \widetilde{\Lambda}_{n}^{\iota}\right)^{\Delta_{K}, \psi=0} \rightarrow\left(D \widehat{\otimes}_{\mathbb{Q}_{p}} \widetilde{\Lambda}_{n}^{\iota}\right)^{\Delta_{K}, \psi=0}
$$

is an isomorphism. In particular, the natural map

$$
C_{\varphi, \gamma_{K}}^{\bullet}\left(D \widehat{\otimes}_{\mathbb{Q}_{p}} \widetilde{\Lambda}_{n}^{l}\right) \rightarrow C_{\psi, \gamma_{K}}^{\bullet}\left(D \widehat{\otimes}_{\mathbb{Q}_{p}} \widetilde{\Lambda}_{n}^{l}\right)
$$

is a quasi-isomorphism.

(2) The complex

$$
C_{\psi}^{\bullet}(D):[D \stackrel{\psi-1}{\longrightarrow} D]
$$

of $\Lambda_{\infty}$-modules concentrated in degree $[1,2]$ calculates $\mathrm{H}_{\mathrm{Iw}}^{q}(K, D)$, i.e., we have functorial isomorphisms of $\Lambda_{\infty}$-modules

$$
\iota_{D}: D^{\psi=1} \stackrel{\sim}{\rightarrow} \mathbf{H}_{\mathrm{Iw}}^{1}(K, D)
$$

and

$$
D /(\psi-1) D \stackrel{\sim}{\rightarrow} \mathbf{H}_{\mathrm{IW}}^{2}(K, D) .
$$

Proof. This is Theorem 2.6 of [32]. 
Remark 3.4. Let $D$ be a $\left(\varphi, \Gamma_{K}\right)$-module over $\mathbf{B}_{\text {rig, } K}^{\dagger}$. Then one has that the structure of the $\mathbb{Q}_{p}\left[\Gamma_{K}\right]$-module on $D$ uniquely extends to a structure of a continuous $\Lambda_{\infty}$-module (see Proposition 2.13 of [10]).

We define $p_{\Delta_{K}}:=\frac{1}{\left|\Delta_{K}\right|} \sum_{g \in \Delta_{K}} g \in \mathbb{Q}\left[\Delta_{K}\right], \log _{0}(a):=\frac{\log (a)}{p^{v_{p}(\log (a))}} \in \mathbb{Z}_{p}^{\times}$for any non-torsion $a \in \mathbb{Z}_{p}^{\times}$. For $q=1$, the isomorphism

$$
\iota_{D}: D^{\psi=1} \stackrel{\sim}{\rightarrow} \mathbf{H}_{\mathrm{Iw}}^{1}(K, D)
$$

is defined as the composition of the following isomorphisms:

$$
\begin{aligned}
& \iota_{D}: D^{\psi=1} \stackrel{\sim}{\rightarrow}{\underset{n}{\lim }}_{n}\left(\left(D \widehat{\otimes}_{\mathbb{Q}_{p}} \widetilde{\Lambda}_{n}^{\iota}\right)^{\Delta_{K}} /\left(\gamma_{K}-1\right)\left(D \widehat{\otimes}_{\mathbb{Q}_{p}} \widetilde{\Lambda}_{n}^{\iota}\right)^{\Delta_{K}}\right)^{\psi=1} \\
& \stackrel{\sim}{\rightarrow} \underset{n}{\lim } \mathrm{H}^{1}\left(C_{\psi, \gamma_{K}}^{\bullet}\left(D \hat{\otimes} \mathbb{Q}_{p} \widetilde{\Lambda}_{n}^{\iota}\right)\right) \\
& \stackrel{\sim}{\rightarrow} \underset{n}{\lim } \mathrm{H}^{1}\left(C_{\varphi, \gamma_{K}}^{\bullet}\left(D \hat{\otimes} \mathbb{Q}_{p} \widetilde{\Lambda}_{n}^{\iota}\right)\right)=\mathbf{H}_{\mathrm{IW}}^{1}(K, D),
\end{aligned}
$$

where each isomorphism is defined as follows. The first isomorphism is defined by $x \mapsto\left(\left|\Gamma_{K \text {,tor }}\right| \log _{0}\left(\chi\left(\gamma_{K}\right)\right) \overline{p_{\Delta_{K}}(x \widehat{\otimes} 1)}\right)_{n \geqq 1}$, for any $x \in D^{\psi=1}$ (see the proof of Theorem 2.6 of [32]). We remark that it is $\Lambda_{\infty}$-linear because we have $p_{\Delta_{K}}(\gamma x \widehat{\otimes} 1)=p_{\Delta_{K}}(x \widehat{\otimes} 1) \cdot[\gamma](\bmod$ $\left.\left(\gamma_{K}-1\right)\right)$ for any $\gamma \in \Gamma_{K}$. The second isomorphism is defined as the projective limit of the isomorphisms

$$
\left(\left(D \widehat{\otimes}_{\mathbb{Q}_{p}} \widetilde{\Lambda}_{n}^{\iota}\right)^{\Delta_{K}} /\left(\gamma_{K}-1\right)\left(D \widehat{\otimes}_{\mathbb{Q}_{p}} \widetilde{\Lambda}_{n}^{\iota}\right)^{\Delta_{K}}\right)^{\psi=1} \stackrel{\sim}{\rightarrow} \mathrm{H}^{1}\left(C_{\psi, \gamma_{K}}^{\bullet}\left(D \widehat{\otimes}_{\mathbb{Q}_{p}} \widetilde{\Lambda}_{n}^{\iota}\right)\right): \bar{x} \mapsto[x, y],
$$

where $x \in\left(D \widehat{\otimes}_{\mathbb{Q}_{p}} \widetilde{\Lambda}_{n}^{\iota}\right)^{\Delta_{K}}$ is a lift of $\bar{x}$ and $y \in\left(D \widehat{\otimes}_{\mathbb{Q}_{p}} \widetilde{\Lambda}_{n}^{\iota}\right)^{\Delta_{K}}$ is an element such that $(\psi-1) x=\left(\gamma_{K}-1\right) y$ (see Proposition 2.4 of [32]). The third isomorphism is induced by Theorem $3.3(1)$.

For each $k \in \mathbb{Z}$, we have the following commutative diagram:

$$
\begin{array}{cc}
D^{\psi=1} \stackrel{\iota_{D}}{\longrightarrow} & \mathbf{H}_{\mathrm{IW}}^{1}(K, D) \\
\downarrow_{x \mapsto x \otimes e_{k}} & \downarrow_{D, k} \\
D(k)^{\psi=1} \stackrel{\iota_{D(k)}}{\longrightarrow} & \mathbf{H}_{\mathrm{IW}}^{1}(K, D(k))
\end{array}
$$

\section{2. $p$-adic differential equations associated to de $\operatorname{Rham}(\varphi, \Gamma)$-modules}

In this subsection, we recall the results of Berger concerning the construction of $p$-adic differential equations associated to de Rham $(\varphi, \Gamma)$-modules. Let $D$ be a de Rham $\left(\varphi, \Gamma_{K}\right)$-module over $\mathbf{B}_{\mathrm{rig}, K}^{\dagger}$. Then we have an isomorphism $K_{n}((t)) \otimes_{K} \mathbf{D}_{\mathrm{dR}}^{K}(D) \stackrel{\sim}{\rightarrow}$ $\mathbf{D}_{\text {dif }, n}(D)$ for each $n \geqq n(D)$. Hence $K_{n}[[t]] \otimes_{K} \mathbf{D}_{\mathrm{dR}}^{K}(D)$ is a $\Gamma_{K}$-stable $K_{n}[[t]]$-lattice of $\mathbf{D}_{\text {dif }, n}(D)$ for each $n \geqq n(D)$. Define $\nabla_{0}:=\frac{\log (\gamma)}{\log (\chi(\gamma))} \in \Lambda_{\infty}$, where $\gamma$ is a non-torsion element of $\Gamma_{K}$, which is independent of the choice of $\gamma$. For each $i \in \mathbb{Z}$, we define $\nabla_{i}:=\nabla_{0}-i \in \Lambda_{\infty}$. The operator $\nabla_{0}$ satisfies the Leibnitz rule $\nabla_{0}(f x)=\nabla_{0}(f) x+f \nabla_{0}(x)$ for any $f \in \mathbf{B}_{\text {rig, } K}^{\dagger}, x \in D$. When $K=F$ is unramified over $\mathbb{Q}_{p}$, then we have $\nabla_{0}(f(T))=$ $t(T+1) \frac{d f(T)}{d T}$ for $f(T) \in \mathbf{B}_{\text {rig, }, F}^{\dagger}$. For the case of general $K$, let $P(X) \in \mathbf{B}_{\mathrm{rig}, K_{0}^{\prime}}^{\dagger}[T]$ be the monic minimal polynomial of $\pi_{K} \in \mathbf{B}_{\text {rig, } K}^{\dagger}$ over $\mathbf{B}_{\text {rig, } K_{0}^{\prime}}^{\dagger}$. Calculating $0=\nabla_{0}\left(P\left(\pi_{K}\right)\right)$, we 
obtain $\nabla_{0}\left(\pi_{K}\right)=-\frac{1}{\frac{d P}{d X}\left(\pi_{K}\right)} \nabla_{0}(P)\left(\pi_{K}\right)$, where we define $\nabla_{0}(P)(X):=\sum_{i=0}^{m} \nabla_{0}\left(a_{i}\right) X^{m}$ for any $P(X)=\sum_{i=0}^{m} a_{m} X^{m} \in B_{\mathrm{rig}, K_{0}^{\prime}}^{\dagger}[X]$. We denote by $\widehat{\Omega}_{\mathbf{B}_{\mathrm{rig}, K}^{\dagger} / K_{0}^{\prime}}$ the continuous differentials. Then one has $\widehat{\Omega}_{\mathbf{B}_{\mathrm{rig}, K}^{\dagger} / K_{0}^{\prime}}=\mathbf{B}_{\text {rig, }, K}^{\dagger} d T$ by the étaleness of the inclusion $\mathbf{B}_{\text {rig, } K_{0}^{\prime}}^{\dagger} \subseteq \mathbf{B}_{\text {rig }, K}^{\dagger}$.

Theorem 3.5. Let $D$ be a de Rham $\left(\varphi, \Gamma_{K}\right)$-module over $\mathbf{B}_{\mathrm{rig}, K}^{\dagger}$ of rank $d$. For each $n \geqq n(D)$, we define

$$
\mathbf{N}_{\text {rig }}^{(n)}(D):=\left\{x \in D^{(n)}[1 / t] \mid \iota_{m}(x) \in K_{m}[[t]] \otimes_{K} \mathbf{D}_{\mathrm{dR}}^{K}(D) \text { for any } m \geqq n\right\} .
$$

Then $\mathbf{N}_{\text {rig }}(D):=\lim _{\rightarrow} \mathbf{N}_{\text {rig }}^{(n)}(D)$ is a $\left(\varphi, \Gamma_{K}\right)$-module over $\mathbf{B}_{\text {rig, }, K}^{\dagger}$ of rank $d$ which satisfies the following.

(1) $\mathbf{N}_{\text {rig }}(D)[1 / t]=D[1 / t]$,

(2) $\mathbf{D}_{\mathrm{dif}, n}^{+}\left(\mathbf{N}_{\text {rig }}(D)\right)=K_{n}[[t]] \otimes_{K} \mathbf{D}_{\mathrm{dR}}^{K}(D)$ for any $n \geqq n(D)$,

(3) $\nabla_{0}\left(\mathbf{N}_{\text {rig }}(D)\right) \subseteq t \mathbf{N}_{\text {rig }}(D)$.

In fact, properties (1) and (2) uniquely characterize $\mathbf{N}_{\text {rig }}(D)$.

Proof. See, for example, Theorem 5.10 of [3] or Theorem 3.2.3 of [6].

By condition (3) in the above theorem, we can define a differential operator

$$
\partial:=\frac{1}{t} \nabla_{0}: \mathbf{N}_{\text {rig }}(D) \rightarrow \mathbf{N}_{\text {rig }}(D)
$$

which satisfies that $\partial \varphi=p \varphi \partial$ and $\partial \gamma=\chi(\gamma) \gamma \partial$ for any $\gamma \in \Gamma_{K}$. In particular, we can equip $\mathbf{N}_{\text {rig }}(D)$ with a structure of a $p$-adic differential equation over $\mathbf{B}_{\text {rig, } K}^{\dagger}$ with Frobenius structure by

$$
\mathbf{N}_{\text {rig }}(D) \rightarrow \mathbf{N}_{\text {rig }}(D) \otimes_{\mathbf{B}_{\text {rig }, K}^{\dagger}} \widehat{\Omega}_{\mathbf{B}_{\text {rig }, K}^{\dagger} / K_{0}^{\prime}}: x \mapsto \partial(x) d T,
$$

where we define $\varphi(d T):=p d T$ and $\gamma(d T):=\chi(\gamma) d T$ for any $\gamma \in \Gamma_{K}$.

Moreover, because we have an isomorphism

$$
\begin{aligned}
\mathbf{N}_{\text {rig }}(D(-1)) & \stackrel{\sim}{\rightarrow} \mathbf{N}_{\text {rig }}(D) \otimes_{\mathbf{B}_{\text {rig }, K}^{\dagger}} \mathbf{N}_{\text {rig }}\left(\mathbf{B}_{\text {rig }, K}^{\dagger}(-1)\right) \\
& =\mathbf{N}_{\text {rig }}(D) \otimes_{\mathbf{B}_{\text {rig }, K}^{\dagger}} t \mathbf{B}_{\text {rig }, K}^{\dagger}(-1)=t \mathbf{N}_{\text {rig }}(D)(-1),
\end{aligned}
$$

we obtain a $\varphi$-equivariant map:

$$
\widetilde{\partial}: \mathbf{N}_{\text {rig }}(D) \rightarrow \mathbf{N}_{\text {rig }}(D(-1)): x \mapsto \nabla_{0}(x) \otimes e_{-1} .
$$

\subsection{Construction of $\operatorname{Exp}_{D, h}$ for de Rham $(\varphi, \Gamma)$-modules}

This subsection is the main part of this article. We generalize Perrin-Riou's big exponential map to all the de $\operatorname{Rham}(\varphi, \Gamma)$-modules, and prove that this map interpolates the exponential map and the dual exponential map of cyclotomic twists of a given $(\varphi, \Gamma)$-module.

We first prove the following easy lemma. We remark that a stronger version (in the crystalline case) appears in $\S 2.2$ of [4]. 
Lemma 3.6. Let $D$ be a de Rham $\left(\varphi, \Gamma_{K}\right)$-module over $\mathbf{B}_{\text {rig, } K}^{\dagger}$, and let $h \in \mathbb{Z}_{\geqq 1}$ such that $\mathrm{Fil}^{-h} \mathbf{D}_{\mathrm{dR}}^{K}(D)=\mathbf{D}_{\mathrm{dR}}^{K}(D)$. Then we have

$$
\nabla_{h-1} \cdot \nabla_{h-2} \cdots \nabla_{1} \cdot \nabla_{0}\left(\mathbf{N}_{\text {rig }}(D)\right) \subseteq D .
$$

Proof. By (3) of Theorem 3.5, and by the formula $\nabla_{i}\left(t^{i} x\right)=t^{i} \nabla_{0}(x)$ for each $i \in \mathbb{Z}$, we obtain an inclusion $\nabla_{h-1} \cdots \nabla_{0}\left(\mathbf{N}_{\text {rig }}(D)\right) \subseteq t^{h} \mathbf{N}_{\text {rig }}(D)$. Hence, it suffices to show that $t^{h} \mathbf{N}_{\text {rig }}(D)$ is contained in $D$. By (2) of Theorem 3.5, we have $\mathbf{D}_{\text {dif }, n}^{+}\left(t^{h} \mathbf{N}_{\text {rig }}(D)\right)=t^{h} K_{n}[[t]] \otimes_{K} \mathbf{D}_{\mathrm{dR}}^{K}(D)$ for each $n \geqq n(D)$. Hence, by the assumption on $h, t^{h} K_{n}[[t]] \otimes_{K} \mathbf{D}_{\mathrm{dR}}^{K}(D)$ is contained in $\operatorname{Fil}^{0}\left(K_{n}((t)) \otimes_{K} \mathbf{D}_{\mathrm{dR}}^{K}(D)\right)=\mathbf{D}_{\mathrm{dif}, n}^{+}(D)$ for any $n \geqq n(D)$. Hence $t^{h} \mathbf{N}_{\text {rig }}(D)$ is also contained in $D$.

Definition 3.7. Let $D$ be a de Rham $\left(\varphi, \Gamma_{K}\right)$-module over $\mathbf{B}_{\text {rig, }, K}^{\dagger}$, and let $h \in \mathbb{Z}_{\geqq 1}$ such that $\mathrm{Fil}^{-h} \mathbf{D}_{\mathrm{dR}}^{K}(D)=\mathbf{D}_{\mathrm{dR}}^{K}(D)$. Then we define a $\Lambda_{\infty}$-linear map

$$
\operatorname{Exp}_{D, h}: \mathbf{N}_{\text {rig }}(D)^{\psi=1} \rightarrow \mathbf{H}_{\mathrm{Iw}}^{1}(K, D): x \mapsto \iota_{D}\left(\nabla_{h-1} \cdots \nabla_{0}(x)\right),
$$

where $\iota_{D}: D^{\psi=1} \stackrel{\sim}{\rightarrow} \mathbf{H}_{\mathrm{Iw}}^{1}(K, D)$ is the isomorphism defined in Theorem 3.3.

Remark 3.8. This definition is strongly influenced by the work of Berger [4], where he reconstructed Perrin-Riou's big exponential map using $(\varphi, \Gamma)$-modules over the Robba ring. Using the work in $\S 3.5$ below, comparing $\mathbf{D}_{\text {crys }}^{K}(D) \otimes_{\mathbb{Q}_{p}}\left(\mathbf{B}_{\text {rig, } \mathbb{Q}_{p}}^{+}\right)^{\psi=0}$ with $\mathbf{N}_{\text {rig }}(D)^{\psi=1}$, one sees that in the crystalline étale case our map is essentially the same as that of Berger, reinterpreted in terms of $\mathbf{N}_{\text {rig }}(D)^{\psi=1}$. Therefore, we regard our map as a generalization of his.

Next, we define a projection map for each $L=K$ or $L=K_{n}(n \geqq 1)$

$$
T_{L}: \mathbf{N}_{\text {rig }}(D)^{\psi=1} \rightarrow \mathbf{D}_{\mathrm{dR}}^{L}(D)
$$

as follows. Because we have $\psi\left(\mathbf{N}_{\text {rig }}^{(m+1)}(D)\right) \subseteq \mathbf{N}_{\text {rig }}^{(m)}(D)$ for any sufficiently large $m$, we have an equality $\mathbf{N}_{\text {rig }}(D)^{\psi=1}=\mathbf{N}_{\text {rig }}^{(m)}(D)^{\psi=1}$ for any $m \gg 0$. Let $n \geqq 1$ be any integer. We take a sufficiently large $m \geqq n$, as above. Then we define $T_{L}$ for $L=K_{n}$ or $L=K$ by

$$
\begin{aligned}
T_{L}: \mathbf{N}_{\text {rig }}(D)^{\psi=1}=\mathbf{N}_{\text {rig }}^{(m)}(D)^{\psi=1} & \stackrel{\iota_{m}}{\rightarrow} K_{m}[[t]] \otimes_{K} \mathbf{D}_{\mathrm{dR}}^{K}(D) \\
& \stackrel{t \mapsto 0}{\longrightarrow} \mathbf{D}_{\mathrm{dR}}^{K_{m}}(D) \stackrel{\frac{1}{\left[K_{m}: L\right]} \operatorname{Tr}_{K_{m} / L}}{\longrightarrow} \mathbf{D}_{\mathrm{dR}}^{L}(D) .
\end{aligned}
$$

Because we have a commutative diagram

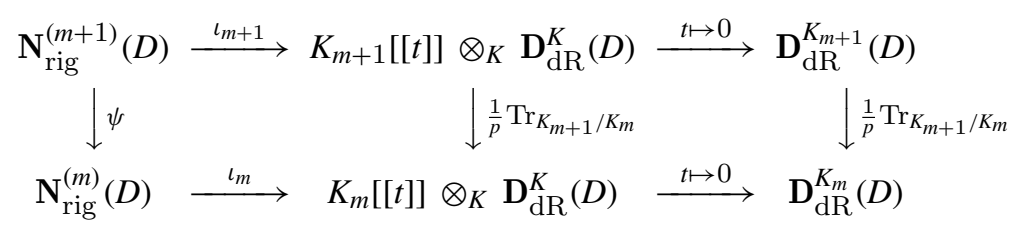

the definition of $T_{L}$ does not depend on the choice of $m \gg n$. 
The following lemma directly follows from the definition.

Lemma 3.9. Let $D$ be a de Rham $\left(\varphi, \Gamma_{K}\right)$-module over $\mathbf{B}_{\text {rig, } K}^{\dagger}$, and let $h \in \mathbb{Z}_{\geqq 1}$ such that $\mathrm{Fil}^{-h} \mathbf{D}_{\mathrm{dR}}^{K}(D)=\mathbf{D}_{\mathrm{dR}}^{K}(D)$. Then we have the following.

(1) $\operatorname{Exp}_{D, h+1}=\nabla_{h} \operatorname{Exp}_{D, h}$.

(2) The following diagram is commutative:

$$
\begin{array}{cr}
\mathbf{N}_{\text {rig }}(D(1))^{\psi=1} \stackrel{\widetilde{\partial}}{\longrightarrow} & \mathbf{N}_{\text {rig }}(D)^{\psi=1} \\
\operatorname{Exp}_{D(1), h+1} & \downarrow \operatorname{Exp}_{D, h} \\
\mathbf{H}_{\mathrm{Iw}}^{1}(K, D(1)) \stackrel{f_{D(1),-1}}{\longrightarrow} & \mathbf{H}_{\mathrm{IW}}^{1}(K, D)
\end{array}
$$

where $f_{D(1),-1}: \mathbf{H}_{\mathrm{Iw}}^{1}(K, D(1)) \stackrel{\sim}{\rightarrow} \mathbf{H}_{\mathrm{Iw}}^{1}(K, D)$ is the canonical isomorphism defined in $\S 3.1$.

The main theorem of this paper is the following, which says that $\operatorname{Exp}_{D, h}$ interpolates $\exp _{L, D(k)}$ for suitable $k \geqq-(h-1)$ and $\exp _{L, D^{\vee}(1-k)}^{*}$ for any $k \leqq-h$ for any $L=K_{n}, K$. According to the comparison of $\mathbf{D}_{\text {crys }}^{K}(D) \otimes \mathbb{Q}_{p}\left(\mathbf{B}_{\text {rig, } \mathbb{Q}_{p}}^{+}\right)^{\psi=0}$ with $\mathbf{N}_{\text {rig }}(D)^{\psi=1}$ provided in $\S 3.5$, we see this theorem as a generalization of Berger's theorem (Theorem 2.10 of [4]) in the crystalline étale case.

Theorem 3.10. For any $h \in \mathbb{Z}_{\geqq 1}$ such that $\mathrm{Fil}^{-h} \mathbf{D}_{\mathrm{dR}}^{K}(D)=\mathbf{D}_{\mathrm{dR}}^{K}(D), \operatorname{Exp}_{D, h}$ satisfies the following formulae.

(1) If $k \geqq 1$ and there exists $x_{k} \in \mathbf{N}_{\text {rig }}(D(k))^{\psi=1}$ such that $\widetilde{\partial}^{k}\left(x_{k}\right)=x$, or if $0 \geqq k \geqq$ $-(h-1)$ and $x_{k}:=\widetilde{\partial}^{-k}(x)$, then

$$
\operatorname{pr}_{L, D(k)}\left(\operatorname{Exp}_{D, h}(x)\right)=\frac{(-1)^{h+k-1}(h+k-1) !\left|\Gamma_{L, \text { tor }}\right|}{p^{m(L)}} \exp _{L, D(k)}\left(T_{L}\left(x_{k}\right)\right),
$$

(2) if $-h \geqq k$, then

$$
\exp _{L, D^{\vee}(1-k)}^{*}\left(\operatorname{pr}_{L, D(k)}\left(\operatorname{Exp}_{D, h}(x)\right)=\frac{\left|\Gamma_{L, \text { tor }}\right|}{(-h-k) ! p^{m(L)}} T_{L}\left(\widetilde{\partial}^{-k}(x)\right),\right.
$$

for any $L=K, K_{n}(n \geqq 1)$, where we put $m(L):=\min \left\{v_{p}\left(\log (\chi(\gamma)) \mid \gamma \in \Gamma_{L}\right\}\right.$.

Proof. We first prove (1). By Lemma 3.9, it suffices to show (1) for $k=0$. Moreover, since we have a commutative diagram

$$
\begin{array}{cc}
\mathbf{D}_{\mathrm{dR}}^{K_{m}}(D) \stackrel{\exp _{K_{m}, D}}{\longrightarrow} & \mathrm{H}^{1}\left(K_{m}, D\right) \\
\downarrow \operatorname{Tr}_{K_{m} / L} & \downarrow \operatorname{cor}_{K_{m} / L} \\
\mathbf{D}_{\mathrm{dR}}^{L}(D) \stackrel{\exp _{L, D}}{\longrightarrow} & \mathrm{H}^{1}(L, D)
\end{array}
$$

for each $L=K_{n}, K(m \geqq n)$ (where $\operatorname{cor}_{K_{m} / L}$ is the corestriction map), and since we have an equality

$$
\left[K_{n}: L\right] \frac{\left|\Gamma_{K_{n}, \text { tor }}\right|}{p^{m\left(K_{n}\right)}}=\frac{\left|\Gamma_{L, \text { tor }}\right|}{p^{m(L)}}
$$


it suffices to show (1) when $L=K_{n}$ for sufficiently large $n$. Hence we may assume that $n \geqq n(D)$ and $\Gamma_{K_{n}}$,tor $=\{1\}$. We set $N_{n}:=\left|\Gamma_{K} /\left(\Gamma_{K_{n}} \times \Delta_{K}\right)\right|$. Then we can write uniquely $\gamma_{K}^{N_{n}}=\gamma_{n} g$, where $\gamma_{n} \in \Gamma_{K_{n}}$ is a topological generator of $\Gamma_{K_{n}}$ and $g \in \Delta_{K}$. Under this situation, we prove (1) using the isomorphisms $\mathbf{H}_{\mathrm{Iw}}^{1}(K, D) \stackrel{\sim}{\rightarrow} \lim _{m} \mathrm{H}^{1}\left(C_{\psi, \gamma_{K}}^{\bullet}\left(D \widehat{\otimes}_{\mathbb{Q}_{p}} \widetilde{\Lambda}_{m}^{\iota}\right)\right)$ and $\mathrm{H}^{1}\left(K_{n}, D\right) \stackrel{\sim}{\rightarrow} \mathrm{H}^{1}\left(C_{\psi, \gamma_{n}}^{\bullet}(D)\right)$. We define

$$
\frac{\nabla_{0}}{\gamma_{n}-1}:=\frac{1}{\log \left(\chi\left(\gamma_{n}\right)\right)} \sum_{k=1}^{\infty} \frac{(-1)^{k-1}}{n}\left(\gamma_{n}-1\right)^{k-1} \in \Lambda_{\infty}
$$

Let $x \in\left(\mathbf{N}_{\text {rig }}^{(n)}(D)\right)^{\psi=1}$ be any element. By the same argument as in the proof of Theorem 2.3 of [4], we have equalities

$$
\frac{\nabla_{0}}{\gamma_{n}-1}\left(T_{K_{n}}(x)\right)=\frac{1}{\log \left(\chi\left(\gamma_{n}\right)\right)} T_{K_{n}}(x) \in \mathbf{D}_{\mathrm{dR}}^{K_{n}}(D)
$$

and

$$
\iota_{n}\left(\frac{\nabla_{0}}{\gamma_{n}-1}(x)\right)=\frac{\nabla_{0}}{\gamma_{n}-1}\left(\iota_{n}(x)\right)=\frac{1}{\log \left(\chi\left(\gamma_{n}\right)\right)} T_{K_{n}}(x)+t z \in K_{n}[[t]] \otimes_{K_{n}} \mathbf{D}_{\mathrm{dR}}^{K_{n}}(D)
$$

for some $z \in K_{n}[[t]] \otimes_{K_{n}} \mathbf{D}_{\mathrm{dR}}^{K_{n}}(D)$. Hence, if we define

$$
\widetilde{x}:=\nabla_{h-1} \cdot \nabla_{h-2} \cdots \nabla_{1} \cdot \frac{\nabla_{0}}{\gamma_{n}-1}(x) \in\left(D^{(n)}[1 / t]\right)^{\psi=1},
$$

then we obtain

$$
\begin{aligned}
\iota_{n}(\tilde{x}) & =\nabla_{h-1} \cdot \nabla_{h-2} \cdots \nabla_{1} \cdot \frac{\nabla_{0}}{\gamma_{n}-1}\left(\iota_{n}(x)\right) \\
& =\nabla_{h-1} \cdot \nabla_{h-2} \cdots \nabla_{1}\left(\frac{1}{\log \left(\chi\left(\gamma_{n}\right)\right)} T_{K_{n}}(x)+t z\right) \\
& \equiv \frac{(-1)^{h-1}(h-1) !}{\log \left(\chi\left(\gamma_{n}\right)\right)} T_{K_{n}}(x)\left(\bmod t^{h} K_{n}[[t]] \otimes_{K_{n}} \mathbf{D}_{\mathrm{dR}}^{K_{n}}(D)\right) .
\end{aligned}
$$

Next, we claim that we have

$$
\iota_{m}(\widetilde{x}) \equiv \iota_{n}(\widetilde{x})\left(\bmod t^{h} K_{m}[[t]] \otimes_{K_{m}} \mathbf{D}_{\mathrm{dR}}^{K_{m}}(D)\right)
$$

for any $m \geqq n$. To prove this claim, it suffices to show that

$$
\iota_{m+1}(\widetilde{x})-\iota_{m}(\widetilde{x}) \in t^{h} K_{m+1}[[t]] \otimes_{K_{m+1}} \mathbf{D}_{\mathrm{dR}}^{K_{m+1}}(D)
$$

for any $m \geqq n$. Moreover, since we have $\iota_{m+1}((\varphi-1) z)=\iota_{m}(z)-\iota_{m+1}(z)$ and $\mathbf{D}_{\text {dif }, m}\left(t^{h} \mathbf{N}_{\text {rig }}(D)\right)=t^{h} K_{m}[[t]] \otimes_{K_{m}} \mathbf{D}_{\mathrm{dR}}^{K_{m}}(D)$, it suffices to show that

$$
(\varphi-1) \widetilde{x} \in t^{h} \mathbf{N}_{\text {rig }}^{(n+1)}(D) \text {. }
$$

Since $x \in \mathbf{N}_{\text {rig }}(D)^{\psi=1}$, we have $\varphi(x)-x \in \mathbf{N}_{\text {rig }}(D)^{\psi=0}$. By Theorem 2.4, there exists a unique $y_{n} \in \mathbf{N}_{\text {rig }}(D)^{\psi=0}$ such that

$$
\varphi(x)-x=\left(\gamma_{n}-1\right) y_{n} .
$$


Then we have

$$
\begin{aligned}
(\varphi-1) \widetilde{x} & \left.=\nabla_{h-1} \cdots \nabla_{1} \cdot \frac{\nabla_{0}}{\gamma_{n}-1}(\varphi(x)-x)\right) \\
& =\nabla_{h-1} \cdots \nabla_{1} \cdot \frac{\nabla_{0}}{\gamma_{n}-1}\left(\left(\gamma_{n}-1\right) y_{n}\right) \\
& =\nabla_{h-1} \cdots \nabla_{1} \cdot \nabla_{0}\left(y_{n}\right) \in t^{h} \mathbf{N}_{\text {rig }}^{(n+1)}(D),
\end{aligned}
$$

where the last inclusion follows from Lemma 3.6.

By this claim, by Lemma 2.12 (1), by the definition of the canonical isomorphism $\mathrm{H}^{1}\left(K_{n}, D\right) \stackrel{\sim}{\rightarrow} \mathrm{H}^{1}\left(C_{\psi, \gamma_{n}}^{\bullet}(D)\right)$, and by the fact that $t^{h} K_{m}[[t]] \otimes_{K_{m}} \mathbf{D}_{\mathrm{dR}}^{K_{m}}(D) \subseteq \mathbf{D}_{\mathrm{dif}, m}^{+}(D)$, we obtain

$$
\begin{aligned}
\frac{(-1)^{h-1}(h-1) !}{\log \left(\chi\left(\gamma_{n}\right)\right)} \exp _{K_{n}, D}\left(T_{K_{n}}(x)\right) & =\left[\left(\gamma_{n}-1\right) \widetilde{x},(1-\psi) \widetilde{x}\right] \\
& =\left[\nabla_{h-1} \cdot \nabla_{h-2} \cdots \nabla_{1} \cdot \nabla_{0}(x), 0\right] \in \mathrm{H}^{1}\left(C_{\psi, \gamma_{n}}^{\bullet}(D)\right) .
\end{aligned}
$$

Since the natural projection map $\operatorname{pr}_{K_{n}, D}: D^{\psi=1} \rightarrow \mathrm{H}^{1}\left(K_{n}, D\right) \stackrel{\sim}{\rightarrow} \mathrm{H}^{1}\left(C_{\psi, \gamma_{n}}^{\bullet}(D)\right)$ is given by $\operatorname{pr}_{K_{n}, D}(y)=\left[\log _{0}\left(\chi\left(\gamma_{n}\right)\right) y, 0\right]$, we obtain

$$
\begin{aligned}
\operatorname{pr}_{K_{n}, D}\left(\operatorname{Exp}_{D, h}(x)\right) & =\left[\log _{0}\left(\chi\left(\gamma_{n}\right)\right) \nabla_{h-1} \cdots \nabla_{0}(x), 0\right] \\
& =(-1)^{h-1}(h-1) ! \frac{\log _{0}\left(\chi\left(\gamma_{n}\right)\right)}{\log \left(\chi\left(\gamma_{n}\right)\right)} \exp _{K_{n}, D}\left(T_{K_{n}}(x)\right) \\
& =\frac{(-1)^{h-1}(h-1) !}{p^{m\left(K_{n}\right)}} \exp _{K_{n}, D}\left(T_{K_{n}}(x)\right),
\end{aligned}
$$

which proves (1).

Next we prove (2). Because we have

$$
\operatorname{Tr}_{K_{n+1} / K_{n}}\left(\exp _{K_{n+1}, D^{\vee}(1)}^{*}(x)\right)=\exp _{K_{n}, D^{\vee}(1)}^{*}\left(\operatorname{cor}_{K_{n+1} / K_{n}}(x)\right)
$$

for any $x \in \mathrm{H}^{1}\left(K_{n+1}, D\right)$, it suffices to show (2) for sufficiently large $n$ as in the proof of (1). Moreover, by Lemma 3.9, it suffices to show (2) for $\operatorname{Exp}_{D, 1}$ when $D$ satisfies $\mathrm{Fil}^{-1} \mathbf{D}_{\mathrm{dR}}^{K}(D)=\mathbf{D}_{\mathrm{dR}}^{K}(D)$. For $x \in \mathbf{N}_{\text {rig }}(D)^{\psi=1}$, we write

$$
\iota_{n}(x):=\sum_{m=0} t^{m} x_{m} \in \mathbf{D}_{\mathrm{dif}, n}^{+}\left(\mathbf{N}_{\mathrm{rig}}(D)\right)=K_{n}[[t]] \otimes_{K_{n}} \mathbf{D}_{\mathrm{dR}}^{K_{n}}(D) \quad\left(x_{m} \in \mathbf{D}_{\mathrm{dR}}^{K_{n}}(D)\right) .
$$

Because we have the following commutative diagram

$$
\begin{aligned}
& \mathbf{N}_{\text {rig }}(D) \longrightarrow \iota_{n} \quad K_{n}[[t]] \otimes_{K_{n}} \mathbf{D}_{\mathrm{dR}}^{K_{n}}(D) \\
& \downarrow^{-k} \quad \downarrow f(t) \otimes x \mapsto\left(\frac{d}{d t}\right)^{-k}(f(t)) \otimes t^{-k} x e_{k} \\
& \mathbf{N}_{\text {rig }}(D(k)) \underset{\iota_{n}}{\longrightarrow} K_{n}[[t]] \otimes_{K_{n}} \mathbf{D}_{\mathrm{dR}}^{K_{n}}(D(k))
\end{aligned}
$$


for each $k \leqq-1$, we obtain

$$
\begin{aligned}
T_{K_{n}}\left(\widetilde{\partial}^{-k}(x)\right) & =\left.\iota_{n}\left(\widetilde{\partial}^{-k}(x)\right)\right|_{t=0} \\
& =\left.\left(\frac{d}{d t}\right)^{-k}\left(\sum_{m=0}^{\infty} t^{m} x_{m}\right)\right|_{t=0} \otimes t^{-k} e_{k} \\
& =(-k) ! \cdot x_{-k} \otimes t^{-k} e_{k} \in \mathbf{D}_{\mathrm{dR}}^{K_{n}}(D(k))=\mathbf{D}_{\mathrm{dR}}^{K_{n}}(D) \otimes t^{-k} e_{k} .
\end{aligned}
$$

On the other hand, we have an equality

$$
\begin{aligned}
\operatorname{pr}_{K_{n}, D(k)}\left(\operatorname{Exp}_{D, 1}(x)\right) & =\operatorname{pr}_{K_{n}, D(k)}\left(\nabla_{0}(x)\right) \\
& =\left[\log _{0}\left(\chi\left(\gamma_{n}\right)\right) \nabla_{0}(x) \otimes e_{k}, 0\right] \in \mathrm{H}^{1}\left(K_{n}, D(k)\right)=\mathrm{H}^{1}\left(C_{\psi, \gamma_{n}}^{\bullet}(D(k))\right),
\end{aligned}
$$

and the natural map

$$
\mathrm{H}^{1}\left(C_{\psi, \gamma_{n}}^{\bullet}(D(k))\right) \rightarrow \mathrm{H}^{1}\left(C_{\gamma_{n}}^{\bullet}\left(\mathbf{D}_{\mathrm{dif}}(D(k))\right)\right)=\mathrm{H}^{1}\left(C_{\gamma_{n}}^{\bullet}\left(K_{\infty}((t)) \otimes_{K_{n}} \mathbf{D}_{\mathrm{dR}}^{K_{n}}(D(k))\right)\right)
$$

sends the element $\left[\log _{0}\left(\chi\left(\gamma_{n}\right)\right) \nabla_{0}(x) \otimes e_{k}, 0\right]$ to

$$
\begin{aligned}
{\left[\log _{0}\left(\chi\left(\gamma_{n}\right)\right) \nabla_{0}\left(\iota_{n}(x)\right) \otimes e_{k}\right] } & =\left[\log _{0}\left(\chi\left(\gamma_{n}\right)\right) \nabla_{0}\left(\sum_{m=0}^{\infty} t^{m} x_{m}\right) \otimes e_{k}\right] \\
& =\left[\log _{0}\left(\chi\left(\gamma_{n}\right)\right)\left(\sum_{m=1}^{\infty} m t^{m} x_{m}\right) \otimes e_{k}\right] .
\end{aligned}
$$

Moreover, since we have

$$
\left[\log _{0}\left(\chi\left(\gamma_{n}\right)\right)\left(\sum_{m=1, m \neq-k}^{\infty} m t^{m} x_{m}\right) \otimes e_{k}\right]=0 \in \mathrm{H}^{1}\left(C_{\gamma_{n}}^{\bullet}\left(\mathbf{D}_{\mathrm{dif}}(D(k))\right)\right)
$$

we obtain an equality

$$
\begin{aligned}
{\left[\log _{0}\left(\chi\left(\gamma_{n}\right)\right)\left(\sum_{m=1}^{\infty} m t^{m} x_{m}\right) \otimes e_{k}\right] } & =\left[\log _{0}\left(\chi\left(\gamma_{n}\right)\right)(-k) x_{-k} t^{-k} \otimes e_{k}\right] \\
& \in \mathrm{H}^{1}\left(C_{\gamma_{n}}^{\bullet}\left(\mathbf{D}_{\text {dif }}(D(k))\right)\right) .
\end{aligned}
$$

By these calculations, and by the definition of $\exp _{K_{n}, D^{\vee}(1-k)}^{*}$, we obtain

$$
\begin{aligned}
\exp _{K_{n}, D^{\vee}(1-k)}^{*}\left(\operatorname{pr}_{K_{n}, D(k)}\left(\operatorname{Exp}_{D, 1}(x)\right)\right) & =\exp _{K_{n}, D^{\vee}(1-k)}^{*}\left(\left[\log _{0}\left(\chi\left(\gamma_{n}\right)\right) \nabla_{0}(x) \otimes e_{k}, 0\right]\right) \\
& =(-k) \frac{\log _{0}\left(\chi\left(\gamma_{n}\right)\right)}{\log \left(\chi\left(\gamma_{n}\right)\right)} x_{-k} \otimes t^{-k} e_{k} \in \mathbf{D}_{\mathrm{dR}}^{K_{n}}(D(k)) \\
& =\frac{1}{(-1-k) ! \cdot p^{m\left(K_{n}\right)}} T_{K_{n}}\left(\widetilde{\partial}^{-k}(x)\right),
\end{aligned}
$$

which proves (2), and hence finishes the proof of the theorem.

\subsection{Determinant of $\operatorname{Exp}_{D, h}$ : a generalization of Perrin-Riou's theorem $\delta(V)$}

In this subsection, we formulate and prove a theorem which we call $\delta(D)$ concerning the determinant of our big exponential maps, which says that the determinant of our map $\operatorname{Exp}_{D, h}$ can be described by the second Iwasawa cohomologies $\mathbf{H}_{\mathrm{Iw}}^{2}(K, D)$ and 
$\mathbf{H}_{\mathrm{Iw}}^{2}\left(K, \mathbf{N}_{\text {rig }}(D)\right)$ and by the ' $\Gamma$-factor', which is determined by the Hodge-Tate weights of $D$.

To formulate theorem $\delta(D)$, we need to recall the definition of the characteristic ideal $\operatorname{char}_{\Lambda_{\infty}}(M) \subseteq \Lambda_{\infty}$ for a co-admissible torsion $\Lambda_{\infty}$-module $M$. A co-admissible $\Lambda_{\infty}$-module is defined as a $\Lambda_{\infty}$-module which is isomorphic to the global section of a coherent sheaf on the rigid analytic space $\cup_{n} \operatorname{Spm}\left(\Lambda_{n}\right)$. See [33] or $\S 1$ of [32] for more precise definitions. Let $M$ be a torsion co-admissible $\Lambda_{\infty}$-module. For each $n \geqq 1$, we put $M_{n}:=M \otimes_{\Lambda_{\infty}} \Lambda_{n}$, which is a finite generated torsion $\Lambda_{n}$-module, and $M \stackrel{\sim}{\rightarrow} \lim _{n} M_{n}$ by the theorem of Schneider and Teitelbaum. Since $\Lambda_{n}$ is a finite product of principal ideal domains, $M_{n}$ is a finite length $\Lambda_{n}$-module. Hence, we can define a unique principal ideal $\left(f_{M_{n}}\right)$ of $\Lambda_{n}$ such that $\operatorname{length}_{\left(\Lambda_{n}\right)_{x}}\left(\left(M_{n}\right)_{x}\right)=v_{x}\left(f_{M_{n}}\right)$ for each maximal ideal $x$ of $\Lambda_{n}$, where $v_{x}$ is the normalized valuation of the local ring $\left(\Lambda_{n}\right)_{x}$ of $\Lambda_{n}$ at $x$. By the theorem of Lazard, there exists a unique principal ideal $\left(f_{M}\right)$ of $\Lambda_{\infty}$ such that $f_{M} \Lambda_{n}=\left(f_{M_{n}}\right) \subseteq \Lambda_{n}$ for each $n \geqq 1$. Then, the characteristic ideal $\operatorname{char}_{\Lambda_{\infty}}(M)$ of $M$ is defined by

$$
\operatorname{char}_{\Lambda_{\infty}}(M):=\left(f_{M}\right) \subseteq \Lambda_{\infty}
$$

Let $\operatorname{Frac}\left(\Lambda_{\infty}\right)$ be the ring of the total fractions of $\Lambda_{\infty}$. Since we have $\Lambda_{\infty} \stackrel{\sim}{\rightarrow} \bigoplus_{\eta \in \widehat{\Gamma}_{K, \text { tor }}} \Lambda_{\infty} \alpha_{\eta}$ and we have a non-canonical isomorphism $\Lambda_{\infty} \alpha_{\eta} \stackrel{\sim}{\rightarrow} \mathbf{B}_{\text {rig, } \mathbb{Q}_{p}}^{+}$for each $\eta \in \widehat{\Gamma}_{K}$,tor, we have $\operatorname{Frac}\left(\Lambda_{\infty}\right)=\bigoplus_{\eta \in \widehat{\Gamma}_{K} \text { tor }} \operatorname{Frac}\left(\Lambda_{\infty} \alpha_{\eta}\right)$, where $\operatorname{Frac}\left(\Lambda_{\infty} \alpha_{\eta}\right)$ is the fraction field of $\Lambda_{\infty} \alpha_{\eta}$. For any principal ideals $\left(f_{1}\right),\left(f_{2}\right) \subseteq \Lambda_{\infty}$ such that $f_{i} \alpha_{\eta} \neq 0$ for any $i=1,2$ and $\eta \in \widehat{\Gamma}_{K}$,tor, we denote by $\left(f_{1}\right)\left(f_{2}\right)^{-1} \subseteq \operatorname{Frac}\left(\Lambda_{\infty}\right)$ the principal fractional ideal of $\operatorname{Frac}\left(\Lambda_{\infty}\right)$ generated by $\frac{f_{1}}{f_{2}} \in \operatorname{Frac}\left(\Lambda_{\infty}\right)$.

Let $M_{1}$ and $M_{2}$ be co-admissible $\Lambda_{\infty}$-modules, and let $f: M_{1} \rightarrow M_{2}$ be a $\Lambda_{\infty}$-linear morphism. We assume that $\operatorname{Coker}(f)$ is a torsion $\Lambda_{\infty}$-module and that the natural induced map $\alpha_{\eta} \bar{f}: \alpha_{\eta}\left(M_{1} / M_{1, \text { tor }}\right) \rightarrow \alpha_{\eta}\left(M_{2} / M_{2, \text { tor }}\right)$ is a non-zero injection for each $\eta \in \widehat{\Gamma}_{K, \text { tor }}$. Because we have $\left(M / M_{\text {tor }}\right) \otimes_{\Lambda_{\infty}} \Lambda_{n} \stackrel{\sim}{\rightarrow} M_{n} / M_{n, \text { tor }}$ for any co-admissible $\Lambda_{\infty}$-module $M$, and because the latter is a finite projective $\Lambda_{n}$-module, we can define $\operatorname{det}_{\Lambda_{n}}\left(\bar{f}_{n}\right):=\operatorname{det}_{\Lambda_{n}}\left(\bar{f}_{n}: M_{1, n} / M_{1, n, \text { tor }} \rightarrow M_{2, n} / M_{2, n \text {,tor }}\right) \in \Lambda_{n}$ and $\operatorname{det}_{\Lambda_{\infty}}(\bar{f}):=$ $\lim _{n} \operatorname{det}_{\Lambda_{n}}\left(\bar{f}_{n}\right) \in \Lambda_{\infty}$, which satisfies that $\alpha_{\eta} \operatorname{det}_{\Lambda_{\infty}}(\bar{f}) \neq 0$ for any $\eta \in \widehat{\Gamma}_{K}$,tor. We define a principal fractional ideal $\operatorname{det}_{\Lambda_{\infty}}(f) \subseteq \operatorname{Frac}\left(\Lambda_{\infty}\right)$ by

$$
\operatorname{det}_{\Lambda_{\infty}}(f):=\left(\operatorname{det}_{\Lambda_{\infty}}(\bar{f})\right) \operatorname{char}_{\Lambda_{\infty}}\left(M_{2, \text { tor }}\right)\left(\operatorname{char}_{\Lambda_{\infty}}\left(M_{1, \text { tor }}\right)\right)^{-1} \subseteq \operatorname{Frac}\left(\Lambda_{\infty}\right) .
$$

Lemma 3.11. $\operatorname{det}_{\Lambda_{\infty}}(-)$ satisfies the following formulae.

(i) $\operatorname{det}_{\Lambda_{\infty}}(f)=\operatorname{char}_{\Lambda_{\infty}}(\operatorname{Coker}(f))\left(\operatorname{char}_{\Lambda_{\infty}}(\operatorname{Ker}(f))\right)^{-1}$.

(ii) For any $f_{1}: M_{1} \rightarrow M_{2}$ and $f_{2}: M_{2} \rightarrow M_{3}$ as above, we have an equality

$$
\operatorname{det}_{\Lambda_{\infty}}\left(f_{2} \circ f_{1}\right)=\operatorname{det}_{\Lambda_{\infty}}\left(f_{1}\right) \operatorname{det}_{\Lambda_{\infty}}\left(f_{2}\right) .
$$

(iii) If we have a commutative diagram

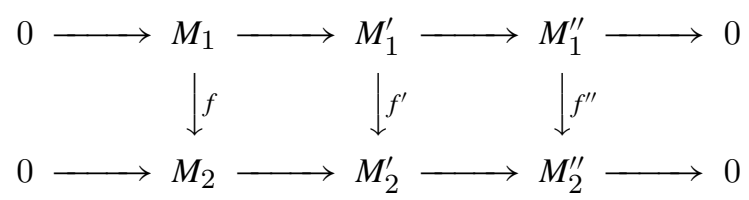


with exact rows, then we have an equality

$$
\operatorname{det}_{\Lambda_{\infty}}\left(f^{\prime}\right)=\operatorname{det}_{\Lambda_{\infty}}(f) \operatorname{det}_{\Lambda_{\infty}}\left(f^{\prime \prime}\right) .
$$

Proof. One can prove this by an easy linear algebra argument, so we omit the proof.

Let $M_{1}, M_{2}$ be $\Lambda_{\infty}$-modules, and let $d_{i}: M_{i} \rightarrow M_{i}$ be a $\Lambda_{\infty}$-linear endomorphism. Denote by $C_{d_{i}}^{\bullet}\left(M_{i}\right):=\left[M_{i} \stackrel{d_{i}}{\rightarrow} M_{i}\right]$ the complex of $\Lambda_{\infty}$-modules concentrated in degree $[1,2]$. We assume that $\mathrm{H}^{j}\left(C_{d_{i}}^{\bullet}\left(M_{i}\right)\right)$ are co-admissible $\Lambda_{\infty}$-modules for any $i, j \in\{1,2\}$. Let $f: M_{1} \rightarrow M_{2}$ be a $\Lambda_{\infty^{-}}$linear morphism which satisfies that $f \circ d_{1}=d_{2} \circ f$. We assume that the induced maps $\mathrm{H}^{i}(f): \mathrm{H}^{i}\left(C_{d_{1}}^{\bullet}\left(M_{1}\right)\right) \rightarrow \mathrm{H}^{i}\left(C_{d_{2}}^{\bullet}\left(M_{2}\right)\right)$ for $i=1,2$ satisfy the conditions in the last paragraph. Then we define a principal fractional ideal

$$
\operatorname{det}_{\Lambda_{\infty}}\left(\mathrm{H}^{\bullet}(f)\right):=\operatorname{det}_{\Lambda_{\infty}}\left(\mathrm{H}^{1}(f)\right) \operatorname{det}_{\Lambda_{\infty}}\left(\mathrm{H}^{2}(f)\right)^{-1} .
$$

Lemma 3.12. $\operatorname{det}_{\Lambda_{\infty}}\left(H^{\bullet}(f)\right)$ satisfies the following.

(iv) For $\left(M_{i}, d_{i}\right)(i=1,2,3), f_{1}: M_{1} \rightarrow M_{2}$ and $f_{2}: M_{2} \rightarrow M_{3}$ as above, we have

$$
\operatorname{det}_{\Lambda_{\infty}}\left(\mathrm{H}^{\bullet}\left(f_{2} \circ f_{1}\right)\right)=\operatorname{det}_{\Lambda_{\infty}}\left(\mathrm{H}^{\bullet}\left(f_{1}\right)\right) \operatorname{det}_{\Lambda_{\infty}}\left(\mathrm{H}^{\bullet}\left(f_{2}\right)\right) .
$$

(v) If $\operatorname{Ker}(f)$ and $\operatorname{Coker}(f)$ are both torsion co-admissible $\Lambda_{\infty}$-modules, then we have an equality

$$
\operatorname{det}_{\Lambda_{\infty}}\left(\mathrm{H}^{\bullet}(f)\right)=\Lambda_{\infty}
$$

Proof. This is also proved by an easy linear algebra argument, so we omit the proof.

We apply these definitions to the following situation. Let $D$ be a de Rham $\left(\varphi, \Gamma_{K}\right)$-module over $\mathbf{B}_{\text {rig, } K}^{\dagger}$. Take $h \geqq 1$ such that $\operatorname{Fil}^{-h} \mathbf{D}_{\mathrm{dR}}^{K}(D)=\mathbf{D}_{\mathrm{dR}}^{K}(D)$. We want to apply the above definitions to the maps $\psi-1: D \rightarrow D, \psi-1: \mathbf{N}_{\text {rig }}(D) \rightarrow \mathbf{N}_{\text {rig }}(D)$ and the map $\nabla_{h-1} \cdots \nabla_{0}: \mathbf{N}_{\text {rig }}(D) \rightarrow D$ defined in Lemma 3.6. By Theorem 3.2, in order to apply the above definition to this setting, we need to show the following lemma.

Lemma 3.13. The map

$$
\overline{\nabla_{h-1} \cdots \nabla_{0}}: \mathbf{N}_{\text {rig }}(D)^{\psi=1} / \mathbf{N}_{\text {rig }}(D)_{\text {tor }}^{\psi=1} \rightarrow D^{\psi=1} / D_{\text {tor }}^{\psi=1}
$$

which is induced by $\nabla_{h-1} \cdots \nabla_{0}: \mathbf{N}_{\text {rig }}(D)^{\psi=1} \rightarrow D^{\psi=1}$ is injective.

Proof. We first note that the map $\nabla_{h-1} \cdots \nabla_{0}: \mathbf{N}_{\text {rig }}(D)^{\psi=1} \rightarrow D^{\psi=1}$ is the composition of $\nabla_{h-1} \cdots \nabla_{0}: \mathbf{N}_{\text {rig }}(D)^{\psi=1} \rightarrow\left(t^{h} \mathbf{N}_{\text {rig }}(D)\right)^{\psi=1}$ with the natural injection $\left(t^{h} \mathbf{N}_{\text {rig }}(D)\right)^{\psi=1} \hookrightarrow D^{\psi=1}$. Since we have

$$
\left(t^{h} \mathbf{N}_{\text {rig }}(D)\right)_{\text {tor }}^{\psi=1}=D_{\text {tor }}^{\psi=1} \cap\left(t^{h} \mathbf{N}_{\text {rig }}(D)\right)^{\psi=1},
$$

the map $\left(t^{h} \mathbf{N}_{\text {rig }}(D)\right)^{\psi=1} /\left(t^{h} \mathbf{N}_{\text {rig }}(D)\right)_{\text {tor }}^{\psi=1} \rightarrow D^{\psi=1} / D_{\text {tor }}^{\psi=1}$ is injective. To show that the map

$$
\mathbf{N}_{\text {rig }}(D)^{\psi=1} / \mathbf{N}_{\text {rig }}(D)_{\text {tor }}^{\psi=1} \stackrel{\overline{\nabla_{h-1} \cdots \nabla_{0}}}{\longrightarrow}\left(t^{h} \mathbf{N}_{\text {rig }}(D)\right)^{\psi=1} /\left(t^{h} \mathbf{N}_{\text {rig }}(D)\right)_{\text {tor }}^{\psi=1}
$$


is injective, it suffices to show that the map

$$
\mathbf{N}_{\text {rig }}(D)^{\psi=1} / \mathbf{N}_{\text {rig }}(D)_{\text {tor }}^{\psi=1} \stackrel{\overline{\nabla_{h-1} \cdots \nabla_{0}}}{\longrightarrow} \mathbf{N}_{\text {rig }}(D)^{\psi=1} / \mathbf{N}_{\text {rig }}(D)_{\text {tor }}^{\psi=1}
$$

is injective. Since $\mathbf{N}_{\text {rig }}(D)^{\psi=1} / \mathbf{N}_{\text {rig }}(D)_{\text {tor }}^{\psi=1}$ is a finite free $\Lambda_{\infty}$-module by Theorem 3.2 and $\nabla_{h-1} \cdots \nabla_{0} \in \Lambda_{\infty}$ is a non-zero divisor, the map

$$
\mathbf{N}_{\text {rig }}(D)^{\psi=1} / \mathbf{N}_{\text {rig }}(D)_{\text {tor }}^{\psi=1} \stackrel{\overline{\nabla_{h-1} \cdots \nabla_{0}}}{\longrightarrow} \mathbf{N}_{\text {rig }}(D)^{\psi=1} / \mathbf{N}_{\text {rig }}(D)_{\text {tor }}^{\psi=1}
$$

is injective, which proves the lemma.

By this lemma and by Theorem 3.2, we can define a fractional ideal

$$
\operatorname{det}_{\Lambda_{\infty}}\left(\mathrm{H}^{\bullet}\left(\mathbf{N}_{\text {rig }}(D) \stackrel{\nabla_{h-1} \cdots \nabla_{0}}{\longrightarrow} D\right)\right) \subseteq \operatorname{Frac}\left(\Lambda_{\infty}\right) .
$$

By the definition of $\operatorname{det}_{\Lambda_{\infty}}(-)$, and since $\mathbf{H}_{\mathrm{IW}}^{2}(K,-)$ are co-admissible torsion $\Lambda_{\infty}$-modules by Theorem 3.2 , we have an equality:

$$
\begin{array}{r}
\operatorname{det}_{\Lambda_{\infty}}\left(\mathrm{H}^{\bullet}\left(\mathbf{N}_{\mathrm{rig}}(D) \stackrel{\nabla_{h-1} \cdots \nabla_{0}}{\longrightarrow} D\right)\right)=\operatorname{det}_{\Lambda_{\infty}}\left(\mathbf{N}_{\mathrm{rig}}(D) \psi=1 \stackrel{\operatorname{Exp}_{D, h}}{\longrightarrow} \mathbf{H}_{\mathrm{Iw}}^{1}(K, D)\right) . \\
\operatorname{char}_{\Lambda_{\infty}}\left(\mathbf{H}_{\mathrm{Iw}}^{2}\left(K, \mathbf{N}_{\mathrm{rig}}(D)\right)\right)\left(\operatorname{char}_{\Lambda_{\infty}}\left(\mathbf{H}_{\mathrm{Iw}}^{2}(K, D)\right)\right)^{-1} .
\end{array}
$$

Concerning this determinant, we have the following theorem. As we will explain in the next subsection, this theorem can be seen as a generalization of theorem $\delta(V)$ of Perrin-Riou (Conjecture 3.4.7 of [29]) and of theorem $\delta(D)$ of Pottharst (Theorem 3.4 of $[32])$.

Theorem 3.14. $(\delta(D))$ Let $D$ be a de Rham $\left(\varphi, \Gamma_{K}\right)$-module over $\mathbf{B}_{\text {rig, },}^{\dagger}$ of rank $d$ with Hodge-Tate weights $\left\{h_{1}, h_{2}, \ldots, h_{d}\right\}$ (note that the Hodge-Tate weight of $\mathbb{Q}_{p}(1)$ is 1 ). For any $h \geqq 1$ such that $\mathrm{Fil}^{-h} \mathbf{D}_{\mathrm{dR}}^{K}(D)=\mathbf{D}_{\mathrm{dR}}^{K}(D)$, we have the following equality of principal fractional ideals of $\Lambda_{\infty}$ :

$$
\begin{aligned}
& \frac{1}{\left(\prod_{i=1}^{d} \prod_{j_{i}=0}^{h-h_{i}-1} \nabla_{h_{i}+j_{i}}\right)^{\left[K: \mathbb{Q}_{p}\right]}} \operatorname{det}_{\Lambda_{\infty}}\left(\mathbf{N}_{\mathrm{rig}}(D)^{\psi=1} \stackrel{\operatorname{Exp}_{D, h}}{\longrightarrow} \mathbf{H}_{\mathrm{Iw}}^{1}(K, D)\right) \\
& =\operatorname{char}_{\Lambda_{\infty}}\left(\mathbf{H}_{\mathrm{Iw}}^{2}(K, D)\right)\left(\operatorname{char}_{\Lambda_{\infty}} \mathbf{H}_{\mathrm{Iw}}^{2}\left(K, \mathbf{N}_{\mathrm{rig}}(D)\right)\right)^{-1} .
\end{aligned}
$$

In particular, the ideal of the left-hand side does not depend on $h$, where we define $\prod_{j_{i}=0}^{h-h_{i}-1} \nabla_{h_{i}+j_{i}}:=1$ when $h=h_{i}$.

Proof. By the definition of $\operatorname{det}_{\Lambda_{\infty}}\left(\mathrm{H}^{\bullet}(-)\right)$ and because we have an isomorphism $\mathrm{H}^{i}([D \stackrel{\psi-1}{\longrightarrow} D]) \stackrel{\sim}{\rightarrow} \mathbf{H}_{\mathrm{Iw}}^{i}(K, D)$ by Theorem 3.3, it suffices to show that

$$
\operatorname{det}_{\Lambda_{\infty}}\left(\mathrm{H}^{\bullet}\left(\mathbf{N}_{\text {rig }}(D) \stackrel{\nabla_{h-1} \cdots \nabla_{0}}{\longrightarrow} D\right)\right)=\left(\prod_{i=1}^{d} \prod_{j_{i}=0}^{h-h_{i}-1} \nabla_{h_{i}+j_{i}}\right)^{\left[K: \mathbb{Q}_{p}\right]}
$$


Moreover, since we have an equality

$$
\begin{aligned}
\operatorname{det}_{\Lambda_{\infty}}\left(\mathrm{H}^{\bullet}\left(\mathbf{N}_{\text {rig }}(D) \stackrel{\nabla_{h-1} \cdots \nabla_{0}}{\longrightarrow} D\right)\right) \\
=\prod_{i=0}^{h-1} \operatorname{det}_{\Lambda_{\infty}}\left(\mathrm{H}^{\bullet}\left(t^{i} \mathbf{N}_{\text {rig }}(D) \stackrel{\nabla_{i}}{\rightarrow} t^{i+1} \mathbf{N}_{\text {rig }}(D)\right)\right) \cdot \operatorname{det}_{\Lambda_{\infty}}\left(\mathrm{H}^{\bullet}\left(t^{h} \mathbf{N}_{\text {rig }}(D) \stackrel{\iota}{\rightarrow} D\right)\right)
\end{aligned}
$$

by Lemma 3.12 (where $\iota: t^{h} \mathbf{N}_{\text {rig }}(D) \hookrightarrow D$ is the canonical inclusion), it suffices to show the following equalities.

(1) $\operatorname{det}_{\Lambda_{\infty}}\left(\mathrm{H}^{\bullet}\left(t^{i} \mathbf{N}_{\text {rig }}(D) \stackrel{\nabla_{i}}{\rightarrow} t^{i+1} \mathbf{N}_{\text {rig }}(D)\right)\right)=\Lambda_{\infty}$ for each $0 \leqq i \leqq h-1$,

$(2) \operatorname{det}_{\Lambda_{\infty}}\left(\mathrm{H}^{\bullet}\left(t^{h} \mathbf{N}_{\text {rig }}(D) \stackrel{\iota}{\rightarrow} D\right)\right)=\left(\prod_{i=1}^{d} \prod_{j_{i}=0}^{h-h_{i}-1} \nabla_{h_{i}+j_{i}}\right)^{\left[K: \mathbb{Q}_{p}\right]}$.

Claim (1) follows from property (v) of Lemma 3.12 because $\operatorname{Ker}\left(\nabla_{i}: t^{i} \mathbf{N}_{\text {rig }}(D) \rightarrow\right.$ $\left.t^{i+1} \mathbf{N}_{\text {rig }}(D)\right)$ and $\operatorname{Coker}\left(\nabla_{i}: t^{i} \mathbf{N}_{\text {rig }}(D) \rightarrow t^{i+1} \mathbf{N}_{\text {rig }}(D)\right)$ are finite-dimensional $K_{0}$-vector spaces by a result of Crew ( $\$ 6$ of [12]) (precisely, his result was under the assumption of the Crew conjecture, which is now a theorem proved by André [1], Mebkhout [24] and Kedlaya [22]).

We prove claim (2) as follows. We first consider the following diagram of short exact sequences:

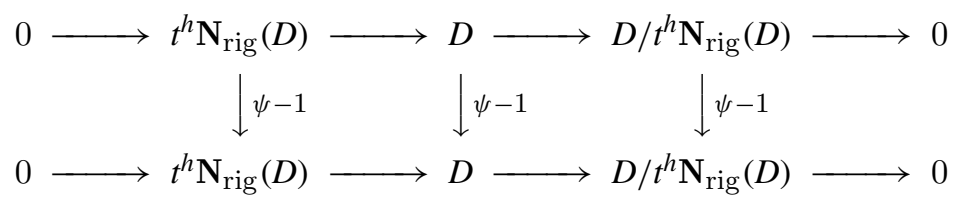

Using the snake lemma, we obtain the following long exact sequence:

$$
\begin{aligned}
0 & \rightarrow \mathbf{H}_{\mathrm{Iw}}^{1}\left(K, t^{h} \mathbf{N}_{\text {rig }}(D)\right) \rightarrow \mathbf{H}_{\mathrm{Iw}}^{1}(K, D) \rightarrow\left(D / t^{h} \mathbf{N}_{\text {rig }}(D)\right)^{\psi=1} \\
& \rightarrow \mathbf{H}_{\mathrm{Iw}}^{2}\left(K, t^{h} \mathbf{N}_{\text {rig }}(D)\right) \rightarrow \mathbf{H}_{\mathrm{IW}}^{2}(K, D) \rightarrow\left(D / t^{n} \mathbf{N}_{\text {rig }}(D)\right) /(\psi-1) \rightarrow 0 .
\end{aligned}
$$

Since $\left(D / t^{h} \mathbf{N}_{\text {rig }}(D)\right)^{\psi=1}$ is a torsion co-admissible $\Lambda_{\infty}$-module and

$$
\left(D / t^{n} \mathbf{N}_{\text {rig }}(D)\right) /(\psi-1)=0
$$

by Proposition 2.1 of [32], we obtain an equality:

$$
\operatorname{det}_{\Lambda_{\infty}}\left(\mathrm{H}^{\bullet}\left(t^{h} \mathbf{N}_{\text {rig }}(D) \stackrel{\iota}{\rightarrow} D\right)\right)=\operatorname{char}_{\Lambda_{\infty}}\left(\left(D / t^{h} \mathbf{N}_{\text {rig }}(D)\right)^{\psi=1}\right) .
$$

Hence, it suffices to show that

$$
\operatorname{char}_{\Lambda_{\infty}}\left(\left(D / t^{h} \mathbf{N}_{\text {rig }}(D)\right)^{\psi=1}\right)=\left(\prod_{i=1}^{d} \prod_{j_{i}=0}^{h-h_{i}-1} \nabla_{h_{i}+j_{i}}\right)^{\left[K: \mathbb{Q}_{p}\right]}
$$

Since we have $\mathbf{D}_{\text {dif }, n}^{+}\left(t^{h} \mathbf{N}_{\text {rig }}(D)\right)=t^{h} K_{n}[[t]] \otimes_{K_{n}} \mathbf{D}_{\mathrm{dR}}^{K_{n}}(D)$ for any sufficiently large $n \gg 0$, we have a $\Lambda_{\infty}$-linear isomorphism for each $n \gg 0$ :

$$
D^{(n)} / t^{h} \mathbf{N}_{\text {rig }}^{(n)}(D) \stackrel{\sim}{\rightarrow} \prod_{m \geqq n} \mathbf{D}_{\text {dif }, m}^{+}(D) /\left(t^{h} K_{m}[[t]] \otimes_{K_{n}} \mathbf{D}_{\mathrm{dR}}^{K_{n}}(D)\right): \bar{x} \mapsto\left(\overline{\iota_{m}(x)}\right)_{m \geqq n},
$$


where the injection follows from the definition of $\mathbf{N}_{\text {rig }}^{(n)}(D)$ and the surjection follows by the same proof as Lemma 2.9. If we write $\mathbf{D}_{\mathrm{dR}}^{K}(D)=\bigoplus_{i=1}^{d} K \beta_{i}$ such that $\beta_{i} \in$ $\mathrm{Fil}^{-h_{i}} \mathbf{D}_{\mathrm{dR}}^{K}(D) \backslash \mathrm{Fil}^{-h_{i}+1} \mathbf{D}_{\mathrm{dR}}^{K}(D)$, then we can write

$$
\begin{aligned}
\mathbf{D}_{\mathrm{dif}, n}^{+}(D) & =\operatorname{Fil}^{0}\left(K_{n}((t)) \otimes_{K_{n}} \mathbf{D}_{\mathrm{dR}}^{K_{n}}(D)\right) \\
& =\bigoplus_{i=1}^{d} K_{n}[[t]]\left(t^{h_{i}} \beta_{i}\right) .
\end{aligned}
$$

Since $\Gamma_{K}$ acts trivially on each $\beta_{i}$, we obtain a $\Lambda_{\infty}$-linear isomorphism:

$$
g_{n}: D^{(n)} / t^{h} \mathbf{N}_{\text {rig }}^{(n)}(D) \stackrel{\sim}{\rightarrow} \bigoplus_{i=1}^{d} \prod_{m \geqq n} t^{h_{i}} K_{m}[[t]] / t^{h} K_{m}[[t]] .
$$

Since we have the following commutative diagrams:

$$
\begin{aligned}
& D^{(n)} / t^{h} \mathbf{N}_{\text {rig }}^{(n)}(D) \quad \underset{g_{n}}{\longrightarrow} \prod_{m \geqq n} \bigoplus_{i=1}^{d} t^{h_{i}} K_{m}[[t]] / t^{h} K_{m}[[t]] \\
& \downarrow \bar{x} \mapsto \bar{x} \quad\left\lfloor\left(x_{m}\right)_{m \geqq n} \mapsto\left(x_{m}\right)_{m \geqq n+1}\right. \\
& D^{(n+1)} / t^{h} \mathbf{N}_{\text {rig }}^{(n+1)}(D) \underset{g_{n+1}}{\longrightarrow} \prod_{m \geqq n+1} \bigoplus_{i=1}^{d} t^{h_{i}} K_{m}[[t]] / t^{h} K_{m}[[t]]
\end{aligned}
$$

and

$$
\begin{array}{ccc}
D^{(n+1)} / t^{h} \mathbf{N}_{\text {rig }}^{(n+1)}(D) & \underset{g_{n+1}}{\longrightarrow} & \prod_{m \geqq n+1} \bigoplus_{i=1}^{d} t^{h_{i}} K_{m}[[t]] / t^{h} K_{m}[[t]] \\
\downarrow \psi & & \downarrow\left(x_{m}\right)_{m \geqq n+1} \mapsto\left(\frac{1}{p} \operatorname{Tr}_{K_{m+1} / K_{m}}\left(x_{m+1}\right)\right)_{m \geqq n} \\
D^{(n)} / t^{h} \mathbf{N}_{\text {rig }}^{(n)}(D) & \underset{g_{n}}{\longrightarrow} & \prod_{m \geqq n} \bigoplus_{i=1}^{d} t^{h_{i}} K_{m}[[t]] / t^{h} K_{m}[[t]]
\end{array}
$$

we obtain the following $\Lambda_{\infty}$-isomorphism:

$$
\begin{aligned}
\left(D / t^{h} \mathbf{N}_{\text {rig }}(D)\right)^{\psi=1} & =\underset{n \gg 0}{\lim _{n \rightarrow 0}}\left(D^{(n)} / t^{h} \mathbf{N}_{\text {rig }}^{(n)}(D)\right)^{\psi=1} \\
& \stackrel{\sim}{\rightarrow} \bigoplus_{i=1}^{d} \underset{n \gg 0}{\lim }(\underbrace{\lim _{m}}_{\frac{1}{p} \operatorname{Tr}_{K_{m+1} / K_{m}}, m \geqq n} t^{h_{i}} K_{m}[[t]] / t^{h} K_{m}[[t]]) \\
& \stackrel{\sim}{\rightarrow} \bigoplus_{i=1}^{d} \underbrace{\lim _{\leftarrow}}_{\frac{1}{p} \operatorname{Tr}_{K_{m+1} / K_{m}}, m \geqq 1} t^{h_{i}} K_{m}[[t]] / t^{h} K_{m}[[t]] .
\end{aligned}
$$

Since we similarly have the $\Lambda_{\infty}$-isomorphism

$$
\left(t^{h^{\prime}} \mathbf{B}_{\text {rig, } K}^{\dagger} / t^{h} \mathbf{B}_{\text {rig, }, K}^{\dagger}\right)^{\psi=1} \stackrel{\sim}{\rightarrow} \underset{\frac{1}{p} \operatorname{Tr}_{K_{m+1} / K_{m}}, m \geqq 1}{\lim } t^{h^{\prime}} K_{m}[[t]] / t^{h} K_{m}[[t]]
$$

for each $h^{\prime} \leqq h$, it suffices to show that

$$
\operatorname{char}_{\Lambda_{\infty}}\left(\left(t^{h^{\prime}} \mathbf{B}_{\text {rig, },}^{\dagger} / t^{h} \mathbf{B}_{\text {rig, },}^{\dagger}\right)^{\psi=1}\right)=\left(\nabla_{h^{\prime}} \nabla_{h^{\prime}-1} \cdots \nabla_{h-1}\right)^{\left[K: \mathbb{Q}_{p}\right]} .
$$


Since we have $\left(t^{h^{\prime}} \mathbf{B}_{\text {rig, } K}^{\dagger} / t^{h} \mathbf{B}_{\text {rig, } K}^{\dagger}\right) /(\psi-1)=0$ for any $h>h^{\prime}$ by Proposition 2.1 of [32], we obtain the following short exact sequence:

$0 \rightarrow\left(t^{h^{\prime}+1} \mathbf{B}_{\text {rig, }, K}^{\dagger} / t^{h} \mathbf{B}_{\text {rig, },}^{\dagger}\right)^{\psi=1} \rightarrow\left(t^{h^{\prime}} \mathbf{B}_{\text {rig, }, K}^{\dagger} / t^{h} \mathbf{B}_{\text {rig }, K}^{\dagger}\right)^{\psi=1} \rightarrow\left(t^{h^{\prime}} \mathbf{B}_{\text {rig, },}^{\dagger} / t^{h^{\prime}+1} \mathbf{B}_{\text {rig, },}^{\dagger}\right)^{\psi=1} \rightarrow 0$

for each $h>h^{\prime}$, and hence we obtain

$$
\operatorname{char}_{\Lambda_{\infty}}\left(\left(t^{h^{\prime}} \mathbf{B}_{\text {rig }, K}^{\dagger} / t^{h} \mathbf{B}_{\text {rig, },}^{\dagger}\right)^{\psi=1}\right)=\prod_{i=0}^{h-h^{\prime}-1} \operatorname{char}_{\Lambda_{\infty}}\left(t^{h^{\prime}+i} \mathbf{B}_{\text {rig }, K}^{\dagger} / t^{h^{\prime}+i+1} \mathbf{B}_{\text {rig, },}^{\dagger}\right)^{\psi=1} .
$$

Hence, to prove claim (2), it suffices to show the following lemma.

Lemma 3.15. For each $h \in \mathbb{Z}$, we have

$$
\operatorname{char}_{\Lambda_{\infty}}\left(\left(t^{h} \mathbf{B}_{\text {rig, } K}^{\dagger} / t^{h+1} \mathbf{B}_{\text {rig }, K}^{\dagger}\right)^{\psi=1}\right)=\left(\nabla_{h}^{\left[K: \mathbb{Q}_{p}\right]}\right) .
$$

Proof. From the short exact sequence

$$
0 \rightarrow t^{h+1} \mathbf{B}_{\mathrm{rig}, K}^{\dagger} \rightarrow t^{h} \mathbf{B}_{\mathrm{rig}, K}^{\dagger} \rightarrow t^{h} \mathbf{B}_{\mathrm{rig}, K}^{\dagger} / t^{h+1} \mathbf{B}_{\mathrm{rig}, K}^{\dagger} \rightarrow 0
$$

and from the fact that $\left(t^{h} \mathbf{B}_{\text {rig, } K}^{\dagger} / t^{h+1} \mathbf{B}_{\text {rig, } K}^{\dagger}\right) /(\psi-1)=0$, we obtain the following exact sequence:

$$
\begin{aligned}
0 & \rightarrow \mathbf{H}_{\mathrm{Iw}}^{1}\left(K, t^{h+1} \mathbf{B}_{\mathrm{rig}, K}^{\dagger}\right) \rightarrow \mathbf{H}_{\mathrm{Iw}}^{1}\left(K, t^{h} \mathbf{B}_{\mathrm{rig}, K}^{\dagger}\right) \rightarrow\left(t^{h} \mathbf{B}_{\mathrm{rig}, K}^{\dagger} / t^{h+1} \mathbf{B}_{\mathrm{rig}, K}^{\dagger}\right)^{\psi=1} \\
& \rightarrow \mathbf{H}_{\mathrm{Iw}}^{2}\left(K, t^{h+1} \mathbf{B}_{\mathrm{rig}, K}^{\dagger}\right) \rightarrow \mathbf{H}_{\mathrm{Iw}}^{2}\left(K, t^{h} \mathbf{B}_{\mathrm{rig}, K}^{\dagger}\right) \rightarrow 0 .
\end{aligned}
$$

Hence, we obtain an equality:

$$
\operatorname{char}_{\Lambda_{\infty}}\left(\left(t^{h} \mathbf{B}_{\text {rig }, K}^{\dagger} / t^{h+1} \mathbf{B}_{\text {rig }, K}^{\dagger}\right)^{\psi=1}\right)=\operatorname{det}_{\Lambda_{\infty}}\left(\mathrm{H}^{\bullet}\left(t^{h+1} \mathbf{B}_{\text {rig }, K}^{\dagger} \rightarrow t^{h} \mathbf{B}_{\text {rig }, K}^{\dagger}\right)\right) .
$$

If we apply (iv) of Lemma 3.12 to the composition of the maps

$$
t^{h} \mathbf{B}_{\mathrm{rig}, K}^{\dagger} \stackrel{\nabla_{h}}{\longrightarrow} t^{h+1} \mathbf{B}_{\mathrm{rig}, K}^{\dagger} \hookrightarrow t^{h} \mathbf{B}_{\mathrm{rig}, K}^{\dagger},
$$

we obtain an equality:

$$
\begin{aligned}
& \operatorname{det}_{\Lambda_{\infty}}\left(\mathrm{H}^{\bullet}\left(t^{h+1} \mathbf{B}_{\mathrm{rig}, K}^{\dagger} \hookrightarrow t^{h} \mathbf{B}_{\mathrm{rig}, K}^{\dagger}\right)\right) \\
& \quad=\operatorname{det}_{\Lambda_{\infty}}\left(\mathrm{H}^{\bullet}\left(t^{h} \mathbf{B}_{\mathrm{rig}, K}^{\dagger} \stackrel{\nabla_{h}}{\longrightarrow} t^{h} \mathbf{B}_{\mathrm{rig}, K}^{\dagger}\right)\right)\left(\operatorname{det}_{\Lambda_{\infty}}\left(\mathrm{H}^{\bullet}\left(t^{h} \mathbf{B}_{\mathrm{rig}, K}^{\dagger} \stackrel{\nabla_{h}}{\longrightarrow} t^{h+1} \mathbf{B}_{\mathrm{rig}, K}^{\dagger}\right)\right)\right)^{-1} .
\end{aligned}
$$

Since we have $\operatorname{det}_{\Lambda_{\infty}}\left(\mathrm{H}^{\bullet}\left(t^{h} \mathbf{B}_{\text {rig, }, K}^{\dagger} \stackrel{\nabla_{h}}{\rightarrow} t^{h+1} \mathbf{B}_{\text {rig, }, K}^{\dagger}\right)\right)=\Lambda_{\infty}$ by claim (1), we obtain

$$
\operatorname{det}_{\Lambda_{\infty}}\left(\mathrm{H}^{\bullet}\left(t^{h+1} \mathbf{B}_{\mathrm{rig}, K}^{\dagger} \hookrightarrow t^{h} \mathbf{B}_{\mathrm{rig}, K}^{\dagger}\right)\right)=\operatorname{det}_{\Lambda_{\infty}}\left(\mathrm{H}^{\bullet}\left(t^{h} \mathbf{B}_{\mathrm{rig}, K}^{\dagger} \stackrel{\nabla_{h}}{\longrightarrow} t^{h} \mathbf{B}_{\mathrm{rig}, K}^{\dagger}\right)\right) .
$$

Finally, because $t^{h} \mathbf{B}_{\text {rig, } K}^{\dagger} /(\psi-1)\left(t^{h} \mathbf{B}_{\text {rig, } K}^{\dagger}\right)$ is a co-admissible torsion $\Lambda_{\infty}$-module and the $\Lambda_{\infty}$-free rank of $\left(t^{h} \mathbf{B}_{\text {rig, } K}^{\dagger}\right)^{\psi=1}$ is $\left[K: \mathbb{Q}_{p}\right]$ by Theorem 3.2, we obtain

$$
\operatorname{det}_{\Lambda_{\infty}}\left(\mathrm{H}^{\bullet}\left(t^{h} \mathbf{B}_{\mathrm{rig}, K}^{\dagger} \stackrel{\nabla_{h}}{\rightarrow} t^{h} \mathbf{B}_{\mathrm{rig}, K}^{\dagger}\right)\right)=\left(\nabla_{h}^{\left[K: \mathbb{Q}_{p}\right]}\right) .
$$

Combining all these equalities, we obtain the equality

$$
\operatorname{char}_{\Lambda_{\infty}}\left(\left(t^{h} \mathbf{B}_{\text {rig, }, K}^{\dagger} / t^{h+1} \mathbf{B}_{\text {rig, }, K}^{\dagger}\right)^{\psi=1}\right)=\left(\nabla_{h}^{\left[K: \mathbb{Q}_{p}\right]}\right),
$$

which proves the lemma, and hence proves the theorem. 


\subsection{Crystalline case}

In this final subsection, we compare our results obtained in the last two subsections with the previous results of Perrin-Riou when $K$ is unramified over $\mathbb{Q}_{p}$ and $D$ is potentially crystalline such that $\left.D\right|_{K_{n}}$ is crystalline for some $n \geqq 0$. After some preliminaries on the theory of $p$-adic Fourier transform, we recall the Berger formula of the Perrin-Riou big exponential map $\Omega_{D, h}[4]$, which is a map from a $\Lambda_{\infty}$-submodule of $\Lambda_{\infty} \otimes_{\mathbb{Q}_{p}} \mathbf{D}_{\text {crys }}^{K_{n}}(D)$ to $\mathbf{H}_{\mathrm{Iw}}^{1}(K, D) / \mathbf{H}_{\mathrm{Iw}}^{1}(K, D)_{\text {tor. }}$. We next recall the statements of Perrin-Riou's theorem $\delta(V)$. Finally, we compare our exponential map $\operatorname{Exp}_{D, h}$ with Perrin-Riou's big exponential map. In particular, we show that our theorem $\delta(D)$ is equivalent to Perrin-Riou's theorem $\delta(V)$ in the unramified and crystalline case.

If $K$ is unramified, the cyclotomic character gives an isomorphism $\chi: \Gamma_{K} \stackrel{\sim}{\rightarrow} \mathbb{Z}_{p}^{\times}$. If we set $T:=[\varepsilon]-1$, then $\mathbf{B}_{\text {rig, } K}^{\dagger}=\cup_{r>0} \mathbf{B}_{\text {rig, }, K}^{\dagger, r}$ can be written as

$$
\mathbf{B}_{\mathrm{rig}, K}^{\dagger, r}:=\left\{f(T):=\sum_{n \in \mathbb{Z}} a_{n} T^{n} \mid a_{n} \in K \text { and } f(T) \text { is convergent on } p^{-1 / r} \leqq|T|_{p}<1\right\},
$$

and the actions of $\varphi$ and $\gamma \in \Gamma_{K}$ are given by the formula

$$
\varphi\left(\sum_{n \in \mathbb{Z}} a_{n} T^{n}\right):=\sum_{n \in \mathbb{Z}} \varphi\left(a_{n}\right)\left((1+T)^{p}-1\right)^{n}, \quad \gamma\left(\sum_{n \in \mathbb{Z}} a_{n} T^{n}\right):=\sum_{n \in \mathbb{Z}} a_{n}\left((1+T)^{\chi(\gamma)}-1\right)^{n} .
$$

We define a $\left(\varphi, \Gamma_{K}\right)$-stable subring $\mathbf{B}_{\text {rig, }, K}^{+}$of $\mathbf{B}_{\text {rig, }, K}^{\dagger}$ by

$$
\mathbf{B}_{\mathrm{rig}, K}^{+}:=\left\{f(T)=\sum_{n=0}^{+\infty} a_{n} T^{n} \mid a_{n} \in K \text { and } f(T) \text { is convergent on } 0 \leqq|T|_{p}<1\right\} .
$$

We have natural $\varphi$ - and $\Gamma_{K} \stackrel{\sim}{\rightarrow} \Gamma_{\mathbb{Q}_{p}}$-equivariant isomorphisms

$$
\mathbf{B}_{\mathrm{rig}, \mathbb{Q}_{p}}^{\dagger} \otimes_{\mathbb{Q}_{p}} K \stackrel{\sim}{\rightarrow} \mathbf{B}_{\mathrm{rig}, K}^{\dagger}, \quad \mathbf{B}_{\mathrm{rig}, \mathbb{Q}_{p}}^{+} \otimes_{\mathbb{Q}_{p}} K \stackrel{\sim}{\rightarrow} \mathbf{B}_{\mathrm{rig}, K}^{+}: f(T) \otimes a \mapsto a f(T) .
$$

One has a $\Lambda_{\infty}$-linear isomorphism defined by

$$
\Lambda_{\infty} \stackrel{\sim}{\rightarrow}\left(\mathbf{B}_{\mathrm{rig}, \mathbb{Q}_{p}}^{+}\right)^{\psi=0}: \lambda \mapsto \lambda \cdot(1+T) .
$$

We remark that the definition of this isomorphism depends on the choice of $T$, i.e., the choice of $\left\{\zeta_{p^{n}}\right\}_{n \geqq 1}$. In this subsection, we consider potentially crystalline $(\varphi, \Gamma)$-modules $D$ over $\mathbf{B}_{\text {rig, } K}^{\dagger}$ such that $\left.D\right|_{K_{n}}$ are crystalline for some $n \geqq 0$.

We first need to study the relationship between $\mathbf{N}_{\text {rig }}(D)^{\psi=1}$ and $\Lambda_{\infty} \otimes_{\mathbb{Q}_{p}} \mathbf{D}_{\text {crys }}^{K_{n}}(D)$.

Lemma 3.16. Let $D$ be a potentially crystalline $\left(\varphi, \Gamma_{K}\right)$-module over $\mathbf{B}_{\mathrm{rig}, K}^{\dagger}$ such that $\left.D\right|_{K_{n}}$ is crystalline for some $n \geqq 0$. Then there exists an isomorphism of $\left(\varphi, \Gamma_{K}\right)$-modules over $\mathbf{B}_{\text {rig, } K}^{\dagger}$ :

$$
\mathbf{N}_{\text {rig }}(D) \stackrel{\sim}{\rightarrow} \mathbf{B}_{\text {rig }, K}^{\dagger} \otimes_{K} \mathbf{D}_{\text {crys }}^{K_{n}}(D),
$$

where, on the right-hand side, $\varphi$ and $\Gamma_{K}$ act diagonally. 
Proof. Since the natural map

$$
\mathbf{B}_{\text {rig }, K}^{\dagger}[1 / t] \otimes_{K} \mathbf{D}_{\text {crys }}^{K_{n}}(D) \rightarrow D[1 / t]: f(T) \otimes x \mapsto f(T) x
$$

is an isomorphism, the natural map

$$
\mathbf{B}_{\text {rig, } K}^{\dagger} \otimes_{K} \mathbf{D}_{\text {crys }}^{K_{n}}(D) \rightarrow D[1 / t]: f(T) \otimes x \mapsto f(T) x
$$

is injective. Then, it is easy to see that $\mathbf{B}_{\text {rig, }, K}^{\dagger} \otimes_{K} \mathbf{D}_{\text {crys }}^{K_{n}}(D) \subseteq D[1 / t]$ satisfies conditions (1) and (2) of Theorem 3.5. Hence $\mathbf{B}_{\text {rig, } K}^{\dagger} \otimes_{K} \mathbf{D}_{\text {crys }}^{K_{n}}(D) \stackrel{\sim}{\rightarrow} \mathbf{N}_{\text {rig }}(D)$ by the uniqueness of $\mathbf{N}_{\text {rig }}(D)$.

By this lemma, $\mathbf{B}_{\text {rig, } K}^{+} \otimes_{K} \mathbf{D}_{\text {crys }}^{K_{n}}(D)$ can be seen as a $\left(\varphi, \Gamma_{K}\right)$-stable submodule of $\mathbf{N}_{\text {rig }}(D)$. Since we have an isomorphism

$$
\Lambda_{\infty} \otimes_{\mathbb{Q}_{p}} \mathbf{D}_{\text {crys }}^{K_{n}}(D) \stackrel{\sim}{\rightarrow}\left(\mathbf{B}_{\text {rig, } \mathbb{Q}_{p}}^{+}\right)^{\psi=0} \otimes_{\mathbb{Q}_{p}} \mathbf{D}_{\text {crys }}^{K_{n}}(D) \stackrel{\sim}{\rightarrow}\left(\mathbf{B}_{\text {rig, }, K}^{+} \otimes_{K} \mathbf{D}_{\text {crys }}^{K_{n}}(D)\right)^{\psi=0}
$$

and the map $(\varphi-1)$ sends $\left(\mathbf{B}_{\text {rig, } K}^{+} \otimes_{K} \mathbf{D}_{\text {crys }}^{K_{n}}(D)\right)^{\psi=1}$ to $\left(\mathbf{B}_{\text {rig, } K}^{+} \otimes_{K} \mathbf{D}_{\text {crys }}^{K_{n}}(D)\right)^{\psi=0}$, to study the relationship between $\Lambda_{\infty} \otimes_{\mathbb{Q}_{p}} \mathbf{D}_{\text {crys }}^{K_{n}}(D)$ and $\mathbf{N}_{\text {rig }}(D)^{\psi=1}$, we need to study the inclusion $\left(\mathbf{B}_{\text {rig }, K}^{+} \otimes_{K} \mathbf{D}_{\text {crys }}^{K_{n}}(D)\right)^{\psi=1} \hookrightarrow \mathbf{N}_{\text {rig }}(D)^{\psi=1}$ and the map

$$
\varphi-1:\left(\mathbf{B}_{\text {rig }, K}^{+} \otimes_{K} \mathbf{D}_{\text {crys }}^{K_{n}}(D)\right)^{\psi=1} \rightarrow\left(\mathbf{B}_{\text {rig }, K}^{+} \otimes_{K} \mathbf{D}_{\text {crys }}^{K_{n}}(D)\right)^{\psi=0} .
$$

Before studying these maps, we recall some facts concerning $p$-adic Fourier transforms (see $\S 2.6$ of $[10]$ ). Let $f: \mathbb{Z}_{p} \rightarrow \mathbb{Q}_{p}$ be a map, and let $h \in \mathbb{Z}_{\geqq 0}$. We say that $f$ is locally $h$-analytic if, for each $x \in \mathbb{Z}_{p}$, there exists $\left\{a_{n}(x)\right\}_{n \geqq 0} \subseteq \mathbb{Q}_{p}$ such that $f\left(x+p^{h} y\right)=\sum_{n=0}^{\infty} a_{n}(x) y^{h}$ for any $y \in \mathbb{Z}_{p}$. We define

$$
\mathrm{LA}_{h}\left(\mathbb{Z}_{p}, \mathbb{Q}_{p}\right):=\left\{f: \mathbb{Z}_{p} \rightarrow \mathbb{Q}_{p} \mid f \text { is locally } h \text {-analytic }\right\}
$$

and

$$
\operatorname{LA}\left(\mathbb{Z}_{p}, \mathbb{Q}_{p}\right):=\underset{h}{\lim } \operatorname{LA} A_{h}\left(\mathbb{Z}_{p}, \mathbb{Q}_{p}\right)
$$

$\mathrm{LA}_{h}\left(\mathbb{Z}_{p}, \mathbb{Q}_{p}\right)$ is a $\mathbb{Q}_{p}$-Banach space whose norm $|-|_{h}$ is defined by

$$
|f|_{h}:=\sup _{x \in \mathbb{Z}_{p}, n \geqq 0}\left|a_{n}(x)\right|_{p} .
$$

We define the actions of $\varphi, \psi$ and $\gamma \in \Gamma_{K} \stackrel{\sim}{\rightarrow} \Gamma_{\mathbb{Q}_{p}}$ on $\operatorname{LA}\left(\mathbb{Z}_{p}, \mathbb{Q}_{p}\right)$ by

$$
\begin{gathered}
\varphi(f)(x):= \begin{cases}0 & \left(\text { if } x \in \mathbb{Z}_{p}^{\times}\right) \\
f\left(\frac{x}{p}\right) & \left(\text { if } x \in p \mathbb{Z}_{p}\right),\end{cases} \\
\psi(f)(x):=f(p x), \quad \gamma(f)(x):=\frac{1}{\chi(\gamma)} f\left(\frac{x}{\chi(\gamma)}\right) .
\end{gathered}
$$

We define a map Col : $\mathbf{B}_{\text {rig, } \mathbb{Q}_{p}}^{\dagger} \rightarrow \mathrm{LA}\left(\mathbb{Z}_{p}, \mathbb{Q}_{p}\right)$, which we call the Colmez transform, by

$$
\operatorname{Col}(f)(x):=\operatorname{Res}\left((1+T)^{x} f(T) \frac{d T}{1+T}\right) \quad \text { for each } x \in \mathbb{Z}_{p},
$$


where Res : $\mathbf{B}_{\text {rig, } \mathbb{Q}_{p}}^{\dagger} \rightarrow \mathbb{Q}_{p}$ is the residue map defined by

$$
\operatorname{Res}\left(\sum_{n \in \mathbb{Z}} a_{n} T^{n}\right):=a_{-1} .
$$

The map Col commutes with the actions of $\psi, \varphi$, and $\Gamma_{\mathbb{Q}_{p}}$, and we have $\operatorname{Ker}(\mathrm{Col})=$ $\mathbf{B}_{\text {rig, } \mathbb{Q}_{p}}^{+}$. Hence we obtain the following short exact sequence:

$$
0 \rightarrow \mathbf{B}_{\text {rig, } \mathbb{Q}_{p}}^{+} \rightarrow \mathbf{B}_{\text {rig, } \mathbb{Q}_{p}}^{\dagger} \stackrel{\mathrm{Col}}{\longrightarrow} \mathrm{LA}\left(\mathbb{Z}_{p}, \mathbb{Q}_{p}\right) \rightarrow 0 .
$$

For each $k \in \mathbb{Z}_{\geq 0}$, we define a locally analytic function $x^{k}: \mathbb{Z}_{p} \rightarrow \mathbb{Q}_{p}: y \mapsto y^{k}$. This function satisfies that

$$
\psi\left(x^{k}\right)=p^{k} x^{k} \quad \text { and } \quad \gamma\left(x^{k}\right)=\chi(\gamma)^{-(k+1)} x^{k}
$$

Lemma 3.17. Let $D_{0}$ be a $\varphi$-module over $\mathbb{Q}_{p}$, i.e., $D_{0}$ is a finite-dimensional $\mathbb{Q}_{p}$-vector space with a $\mathbb{Q}_{p}$-linear automorphism $\varphi: D_{0} \stackrel{\sim}{\rightarrow} D_{0}$. Then, for sufficiently large $k_{0} \gg 0$, we have the following equalities.

(1) $\bigoplus_{k=0}^{k_{0}}\left(t^{k} \otimes D_{0}\right)^{\varphi=1}=\bigoplus_{k=0}^{\infty}\left(t^{k} \otimes D_{0}\right)^{\varphi=1}=\left(\mathbf{B}_{\text {rig, }, \mathbb{Q}_{p}}^{+} \otimes_{\mathbb{Q}_{p}} D_{0}\right)^{\varphi=1}$,

$$
\begin{aligned}
\bigoplus_{k=0}^{k_{0}}\left(t^{k} \otimes D_{0}\right) /(1-\varphi)\left(t^{k} \otimes D_{0}\right) & =\bigoplus_{k=0}^{\infty}\left(t^{k} \otimes D_{0}\right) /(1-\varphi)\left(t^{k} \otimes D_{0}\right) \\
& \stackrel{\sim}{\rightarrow}\left(\mathbf{B}_{\mathrm{rig}, \mathbb{Q}_{p}}^{+} \otimes_{\mathbb{Q}_{p}} D_{0}\right) /(1-\varphi)\left(\mathbf{B}_{\mathrm{rig}, \mathbb{Q}_{p}}^{+} \otimes_{\mathbb{Q}_{p}} D_{0}\right),
\end{aligned}
$$

(3) $\left(\mathbf{B}_{\text {rig, } \mathbb{Q}_{p}}^{+} \otimes_{\mathbb{Q}_{p}} D_{0}\right) /(1-\psi)\left(\mathbf{B}_{\text {rig, } \mathbb{Q}_{p}}^{+} \otimes_{\mathbb{Q}_{p}} D_{0}\right)=0$,

(4) $\bigoplus_{k=0}^{k_{0}}\left(x^{k} \otimes D_{0}\right)^{\psi=1}=\bigoplus_{k=0}^{\infty}\left(x^{k} \otimes D_{0}\right)^{\psi=1}=\left(\operatorname{LA}\left(\mathbb{Z}_{p}, \mathbb{Q}_{p}\right) \otimes_{\mathbb{Q}_{p}} D_{0}\right)^{\psi=1}$,

(5)

$$
\begin{aligned}
\bigoplus_{k=0}^{k_{0}}\left(x^{k} \otimes D_{0}\right) /(1-\psi)\left(x^{k} \otimes D_{0}\right) & =\bigoplus_{k=0}^{\infty}\left(x^{k} \otimes D_{0}\right) /(1-\psi)\left(x^{k} \otimes D_{0}\right) \\
& \stackrel{\sim}{\rightarrow}\left(\operatorname{LA}\left(\mathbb{Z}_{p}, \mathbb{Q}_{p}\right) \otimes_{\mathbb{Q}_{p}} D_{0}\right) /(1-\psi)\left(\operatorname{LA}\left(\mathbb{Z}_{p}, \mathbb{Q}_{p}\right) \otimes_{\mathbb{Q}_{p}} D_{0}\right),
\end{aligned}
$$

where we define $t^{k} \otimes D_{0}:=\mathbb{Q}_{p} t^{k} \otimes_{\mathbb{Q}_{p}} D_{0}$ and $x^{k} \otimes D_{0}:=\mathbb{Q}_{p} x^{k} \otimes_{\mathbb{Q}_{p}} D_{0}$ for each $k \geqq 0$.

Proof. When $D_{0}$ is one dimensional, then all these properties are proved in $\S 2$ of [10]. In the general case, this lemma can be proved in the same way, so we omit the proof.

We go back to our situation. Let $D$ be a potentially crystalline $\left(\varphi, \Gamma_{K}\right)$-module over $\mathbf{B}_{\text {rig, } K}^{\dagger}$ such that $\left.D\right|_{K_{n}}$ is crystalline for some $n \geqq 0$. We define a $\Lambda_{\infty}$-linear morphism

$$
\widetilde{\Delta}:\left(\mathbf{B}_{\mathrm{rig}, K}^{+} \otimes_{K} \mathbf{D}_{\mathrm{crys}}^{K_{n}}(D)\right)^{\psi=0} \rightarrow \bigoplus_{k=0}^{\infty} t^{k} \otimes \mathbf{D}_{\mathrm{crys}}^{K_{n}}(D) /(1-\varphi)\left(t^{k} \otimes \mathbf{D}_{\mathrm{crys}}^{K_{n}}(D)\right)
$$


by

$$
\left.\widetilde{\Delta}\left(\sum_{i=1}^{m} f_{i}(T) \otimes z_{i}\right):=\overline{\left(t^{k} \otimes\left(\sum_{i=1}^{m} \partial^{k}\left(f_{i}\right)(0) \cdot z_{i}\right)\right.}\right)_{k \geqq 0},
$$

where we recall that $\partial(f)(T)=(1+T) \frac{d f(T)}{d T}$.

The following lemma was proved in $\S 2.2$ of [29], but here we reprove it using the above lemma.

Lemma 3.18. There exists the following exact sequence of $\Lambda_{\infty}$-modules:

$$
\begin{gathered}
0 \rightarrow \bigoplus_{k=0}^{\infty}\left(t^{k} \otimes \mathbf{D}_{\text {crys }}^{K_{n}}(D)\right)^{\varphi=1} \rightarrow\left(\mathbf{B}_{\text {rig }, K}^{+} \otimes_{K} \mathbf{D}_{\text {crys }}^{K_{n}}(D)\right)^{\psi=1} \\
\stackrel{\varphi-1}{\longrightarrow}\left(\mathbf{B}_{\mathrm{rig}, K}^{+} \otimes_{K} \mathbf{D}_{\mathrm{crys}}^{K_{n}}(D)\right)^{\psi=0} \stackrel{\widetilde{\Delta}}{\rightarrow} \bigoplus_{k=0}^{\infty}\left(t^{k} \otimes \mathbf{D}_{\text {crys }}^{K_{n}}(D)\right) /(1-\varphi)\left(t^{k} \otimes \mathbf{D}_{\text {crys }}^{K_{n}}(D)\right) \rightarrow 0 .
\end{gathered}
$$

Proof. Since we have an inclusion

$$
(1-\varphi)\left(\mathbf{B}_{\text {rig, } K}^{+} \otimes_{K} \mathbf{D}_{\text {crys }}^{K_{n}}(D)\right)^{\psi=1} \subseteq\left(\mathbf{B}_{\text {rig }, K}^{+} \otimes_{K} \mathbf{D}_{\text {crys }}^{K_{n}}(D)\right)^{\psi=0},
$$

we have the following exact sequence:

$$
\begin{gathered}
0 \rightarrow \bigoplus_{k=0}^{\infty}\left(t^{k} \otimes \mathbf{D}_{\text {crys }}^{K_{n}}(D)\right)^{\varphi=1} \rightarrow\left(\mathbf{B}_{\text {rig }, K}^{+} \otimes_{K} \mathbf{D}_{\text {crys }}^{K_{n}}(D)\right)^{\psi=1} \\
\stackrel{1-\varphi}{\longrightarrow}\left(\mathbf{B}_{\text {rig }, K}^{+} \otimes_{K} \mathbf{D}_{\text {crys }}^{K_{n}}(D)\right)^{\psi=0} \rightarrow\left(\mathbf{B}_{\text {rig, },}^{+} \otimes_{K} \mathbf{D}_{\text {crys }}^{K_{n}}(D)\right) /(1-\varphi)\left(\mathbf{B}_{\text {rig, },}^{+} \otimes_{K} \mathbf{D}_{\text {crys }}^{K_{n}}(D)\right),
\end{gathered}
$$

where the exactness at the second arrow follows from the equality

$$
\bigoplus_{k=0}^{\infty}\left(t^{k} \otimes \mathbf{D}_{\text {crys }}^{K_{n}}(D)\right)^{\varphi=1}=\left(\mathbf{B}_{\text {rig }, K}^{+} \otimes_{K} \mathbf{D}_{\text {crys }}^{K_{n}}(D)\right)^{\varphi=1}
$$

which is proved in (1) of Lemma 3.17. We show that the natural map

$$
\left(\mathbf{B}_{\text {rig }, K}^{+} \otimes_{K} \mathbf{D}_{\text {crys }}^{K_{n}}(D)\right)^{\psi=0} \rightarrow\left(\mathbf{B}_{\text {rig }, K}^{+} \otimes_{K} \mathbf{D}_{\text {crys }}^{K_{n}}(D)\right) /(1-\varphi)\left(\mathbf{B}_{\text {rig, } K}^{+} \otimes_{K} \mathbf{D}_{\text {crys }}^{K_{n}}(D)\right): z \mapsto \bar{z}
$$

is a surjection. To prove this claim, let $z$ be an element of $\mathbf{B}_{\text {rig, } K}^{+} \otimes_{K} \mathbf{D}_{\text {crys }}^{K_{n}}(D)$. Then it suffices to show that there exists $y \in \mathbf{B}_{\text {rig, } K}^{+} \otimes_{K} \mathbf{D}_{\text {crys }}^{K_{n}}(D)$ such that $\psi(z-(1-\varphi) y)=0$. Because we have $\psi(z-(1-\varphi) y)=\psi(z)-(\psi-1) y$, such $y$ exists by (3) of Lemma 3.17.

By this claim, and because we have a natural isomorphism

$$
\begin{aligned}
& \bigoplus_{k=0}^{\infty} t^{k} \otimes \mathbf{D}_{\text {crys }}^{K_{n}}(D) /(1-\varphi)\left(t^{k} \otimes \mathbf{D}_{\text {crys }}^{K_{n}}(D)\right) \\
& \stackrel{\sim}{\rightarrow}\left(\mathbf{B}_{\text {rig }, K}^{+} \otimes_{K} \mathbf{D}_{\text {crys }}^{K_{n}}(D)\right) /(1-\varphi)\left(\mathbf{B}_{\text {rig }, K}^{+} \otimes_{K} \mathbf{D}_{\text {crys }}^{K_{n}}(D)\right)
\end{aligned}
$$

by Lemma 3.17, we obtain the surjection

$$
\left(\mathbf{B}_{\text {rig }, K}^{+} \otimes_{K} \mathbf{D}_{\text {crys }}^{K_{n}}(D)\right)^{\psi=0} \rightarrow \bigoplus_{k=0}^{\infty}\left(t^{k} \otimes \mathbf{D}_{\text {crys }}^{K_{n}}(D)\right) /(1-\varphi)\left(t^{k} \otimes \mathbf{D}_{\text {crys }}^{K_{n}}(D)\right),
$$


which is explicitly defined by

$$
\sum_{i=1}^{m} f_{i}(T) \otimes x_{i} \mapsto\left(\frac{1}{k !} t^{k} \otimes\left(\sum_{i=1}^{m} \partial^{k}\left(f_{i}\right)(0) \cdot x_{i}\right)\right)_{k \geqq 0} .
$$

Since this map and $\widetilde{\Delta}$ only differ by a factor of $k$ ! at each $k$ th component, their kernels and images are equal. Hence we finish proving the exactness of the sequence in this lemma.

The following definition is Berger's formula for Perrin-Riou's big exponential map. More precisely, Berger defined Perrin-Riou's map for crystalline $p$-adic representations, and the following definition is just the direct generalization of his formula for potentially crystalline $(\varphi, \Gamma)$-modules.

Definition 3.19. Let $D$ be a potentially crystalline $\left(\varphi, \Gamma_{K}\right)$-module over $\mathbf{B}_{\text {rig, } K}^{\dagger}$ such that $\left.D\right|_{K_{n}}$ is crystalline for some $n \geqq 0$, and let $h \geqq 1$ be an integer such that Fil $^{-k} \mathbf{D}_{\mathrm{dR}}^{K}(D)=\mathbf{D}_{\mathrm{dR}}^{K}(D)$. Then, we define a $\Lambda_{\infty}$-linear map

$$
\Omega_{D, h}:\left(\Lambda_{\infty} \otimes_{\mathbb{Q}_{p}} \mathbf{D}_{\text {crys }}^{K_{n}}(D)\right)^{\widetilde{\Delta}=0} \rightarrow \mathbf{H}_{\mathrm{Iw}}^{1}(K, D) / \mathbf{H}_{\mathrm{Iw}}^{1}(K, D)_{\text {tor }}
$$

as the composition of the isomorphism

$$
(\varphi-1)^{-1}:\left(\Lambda_{\infty} \otimes_{\mathbb{Q}_{p}} \mathbf{D}_{\text {crys }}^{K_{n}}(D)\right)^{\widetilde{\Delta}=0} \stackrel{\sim}{\rightarrow}\left(\mathbf{B}_{\text {rig }, K}^{+} \otimes_{K} \mathbf{D}_{\text {crys }}^{K_{n}}(D)\right)^{\psi=1} /\left(\mathbf{B}_{\text {rig }, K}^{+} \otimes_{K} \mathbf{D}_{\text {crys }}^{K_{n}}(D)\right)^{\varphi=1}
$$

with the natural inclusion

$$
\left(\mathbf{B}_{\text {rig }, K}^{+} \otimes_{K} \mathbf{D}_{\text {crys }}^{K_{n}}(D)\right)^{\psi=1} /\left(\mathbf{B}_{\text {rig }, K}^{+} \otimes_{K} \mathbf{D}_{\text {crys }}^{K_{n}}(D)\right)^{\varphi=1} \hookrightarrow \mathbf{N}_{\text {rig }}(D)^{\psi=1} / \mathbf{N}_{\text {rig }}(D)^{\varphi=1}
$$

and with the injection proved in Lemma 3.13

$$
\overline{\operatorname{Exp}}_{D, h}: \mathbf{N}_{\text {rig }}(D)^{\psi=1} / \mathbf{N}_{\text {rig }}(D)^{\varphi=1} \hookrightarrow \mathbf{H}_{\mathrm{Iw}}^{1}(K, D) / \mathbf{H}_{\mathrm{IW}}^{1}(K, D)_{\text {tor }}
$$

Remark 3.20. Let $V$ be a crystalline representation of $G_{K}$, and let $D(V)$ be the $\left(\varphi, \Gamma_{K}\right)$-module over $\mathbf{B}_{\text {rig, } K}^{\dagger}$ associated to $V$. If we admit the natural isomorphisms $\Lambda_{\infty} \otimes{ }_{\Lambda} \mathbf{H}_{\mathrm{Iw}}^{1}(K, V) \stackrel{\sim}{\rightarrow} \mathbf{H}_{\mathrm{Iw}}^{1}(K, D) \quad($ see $\S 2$ of $[32])$ and $\mathbf{D}_{\text {crys }}^{K}(V) \stackrel{\sim}{\rightarrow} \mathbf{D}_{\text {crys }}^{K}(D(V))$, Berger proved that the map

$$
\begin{aligned}
& \Omega_{V, h}:\left(\Lambda_{\infty} \otimes_{\mathbb{Q}_{p}} \mathbf{D}_{\mathrm{crys}}^{K}(V)\right)^{\widetilde{\Delta}=0} \stackrel{\sim}{\rightarrow}\left(\Lambda_{\infty} \otimes_{\mathbb{Q}_{p}} \mathbf{D}_{\mathrm{crys}}^{K}(D(V))\right)^{\widetilde{\Delta}=0} \\
& \stackrel{\Omega_{D, h}}{\longrightarrow} \mathbf{H}_{\mathrm{Iw}}^{1}(K, D(V)) / \mathbf{H}_{\mathrm{Iw}}^{1}(K, D(V))_{\mathrm{tor}} \stackrel{\sim}{\rightarrow} \Lambda_{\infty} \otimes_{\Lambda}\left(\mathbf{H}_{\mathrm{Iw}}^{1}(K, V) / \mathbf{H}_{\mathrm{Iw}}^{1}(K, V)_{\text {tor }}\right)
\end{aligned}
$$

coincides with Perrin-Riou's original map defined in [29] (see Theorem 2.13 of [4]).

To state Perrin-Riou's theorem $\delta(V)$, we slightly generalize the definition of $\operatorname{det}_{\Lambda_{\infty}}(-)$ to the following situation. Let $M_{1}$ and $M_{2}$ be co-admissible $\Lambda_{\infty}$-modules. We assume that there exist co-admissible $\Lambda_{\infty}$-submodules $M_{1}^{\prime} \subseteq M_{1}$ and $M_{2}^{\prime} \subseteq M_{2}$ such that $M_{1} / M_{1}^{\prime}$ and $M_{2}^{\prime}$ are torsion $\Lambda_{\infty}$-modules and that there exists a $\Lambda_{\infty}$-linear map $f: M_{1}^{\prime} \rightarrow M_{2} / M_{2}^{\prime}$ for which we can $\operatorname{define}_{\operatorname{det}} \Lambda_{\infty}(f)$. Under this situation, we define a fractional ideal $\operatorname{det}_{\Lambda_{\infty}}\left(f: M_{1} \rightarrow M_{2}\right) \subseteq \operatorname{Frac}\left(\Lambda_{\infty}\right)$ by

$$
\operatorname{det}_{\Lambda_{\infty}}\left(f: M_{1} \rightarrow M_{2}\right):=\operatorname{det}_{\Lambda_{\infty}}\left(f: M_{1}^{\prime} \rightarrow M_{2} / M_{2}^{\prime}\right) \operatorname{char}_{\Lambda_{\infty}}\left(M_{1} / M_{1}^{\prime}\right)^{-1} \operatorname{char}_{\Lambda_{\infty}}\left(M_{2}^{\prime}\right) \text {. }
$$


We apply this definition to the map

$$
\Omega_{D, h}:\left(\Lambda_{\infty} \otimes_{\mathbb{Q}_{p}} \mathbf{D}_{\text {crys }}^{K_{n}}(D)\right)^{\widetilde{\Delta}=0} \rightarrow \mathbf{H}_{\mathrm{Iw}}^{1}(K, D) / \mathbf{H}_{\mathrm{Iw}}^{1}(K, D)_{\text {tor }},
$$

i.e., we define the principal fractional ideal

$$
\operatorname{det}_{\Lambda_{\infty}}\left(\Omega_{D, h}: \Lambda_{\infty} \otimes_{\mathbb{Q}_{p}} \mathbf{D}_{\text {crys }}^{K_{n}}(D) \rightarrow \mathbf{H}_{\mathrm{Iw}}^{1}(K, D)\right)
$$

by the product

$$
\begin{gathered}
\operatorname{det}_{\Lambda_{\infty}}\left(\Omega_{D, h}:\left(\Lambda_{\infty} \otimes_{\mathbb{Q}_{p}} \mathbf{D}_{\text {crys }}^{K_{n}}(D)\right)^{\widetilde{\Delta}=0} \rightarrow \mathbf{H}_{\mathrm{Iw}}^{1}(K, D) / \mathbf{H}_{\mathrm{Iw}}^{1}(K, D)_{\text {tor }}\right) . \\
\operatorname{char}_{\Lambda_{\infty}}\left(\Lambda_{\infty} \otimes_{\mathbb{Q}_{p}} \mathbf{D}_{\text {crys }}^{K_{n}}(D) /\left(\Lambda_{\infty} \otimes_{\mathbb{Q}_{p}} \mathbf{D}_{\text {crys }}^{K_{n}}(D)\right)^{\widetilde{\Delta}=0}\right)^{-1} \cdot \operatorname{char}_{\Lambda_{\infty}}\left(\mathbf{H}_{\mathrm{Iw}}^{1}(K, D)_{\text {tor }}\right) .
\end{gathered}
$$

Using this definition, Perrin-Riou's theorem $\delta(V)$ can be stated as follows. More precisely, the following is the direct generalization of Perrin-Riou's theorem $\delta(V)$ to non-étale crystalline $D$. In Proposition 3.23 below, we will prove the theorem by proving that the theorem is equivalent to Theorem 3.14 .

Theorem 3.21. Let $D$ be a potentially crystalline $\left(\varphi, \Gamma_{K}\right)$-module over $\mathbf{B}_{\text {rig, } K}^{\dagger}$ such that $\left.D\right|_{K_{n}}$ is crystalline. Let $\left\{h_{1}, \ldots, h_{d}\right\}$ be the set of Hodge-Tate weights of $D$, and let $h \geqq 1$ be an integer such that $\mathrm{Fil}^{-h} \mathbf{D}_{\mathrm{dR}}^{K}(D)=\mathbf{D}_{\mathrm{dR}}^{K}(D)$. Then, we have an equality of fractional ideals of $\operatorname{Frac}\left(\Lambda_{\infty}\right)$

$$
\begin{aligned}
\operatorname{det} & \left(\Omega_{D, h}: \Lambda_{\infty} \otimes_{\mathbb{Q}_{p}} \mathbf{D}_{\mathrm{crys}}^{K_{n}}(D) \rightarrow \mathbf{H}_{\mathrm{Iw}}^{1}(K, D)\right) \\
= & \left(\prod_{1 \leqq i \leqq d} \nabla_{h_{i}} \nabla_{h_{i}+1} \cdots \nabla_{h-1}\right)^{\left[K: \mathbb{Q}_{p}\right]} \cdot \operatorname{char}_{\Lambda_{\infty}}\left(\mathbf{H}_{\mathrm{Iw}}^{2}(K, D)\right) .
\end{aligned}
$$

Remark 3.22. On the other hand, for any slope potentially crystalline $D$, Pottharst defined the 'inverse' map

$$
\log _{D}: \mathbf{H}_{\mathrm{Iw}}^{1}(K, D) \rightarrow \operatorname{Frac}\left(\Lambda_{\infty}\right) \otimes_{\mathbb{Q}_{p}} \mathbf{D}_{\text {crys }}^{K_{n}}(D)
$$

of $\Omega_{D, h}$ using the theory of Wach modules. Using $\log _{D}$, he also proved his theorem $\delta(D)$ (Theorem 3.4 of [32]) by reducing it to Perrin-Riou's theorem $\delta(V)$ using a slope filtration argument. It is easy to check that the theorem above is equivalent to his theorem $\delta(D)$.

The next proposition is the main result of this subsection, which says that, when $D$ is as above, our Theorem 3.14 is equivalent to the above Theorem 3.21.

Proposition 3.23. We have an equality

$$
\begin{array}{r}
\operatorname{det}_{\Lambda_{\infty}}\left(\mathbf{N}_{\text {rig }}(D) \psi=1\right. \\
\left.\stackrel{\operatorname{Exp}_{D, h}}{\longrightarrow} \mathbf{H}_{\mathrm{IW}}^{1}(K, D)\right) \cdot \operatorname{char}_{\Lambda_{\infty}}\left(\mathbf{H}_{\mathrm{IW}}^{2}\left(K, \mathbf{N}_{\text {rig }}(D)\right)\right) \\
=\operatorname{det}_{\Lambda_{\infty}}\left(\Lambda_{\infty} \otimes_{\mathbb{Q}_{p}} \mathbf{D}_{\text {crys }}^{K_{n}}(D) \stackrel{\Omega_{D, h}}{\longrightarrow} \mathbf{H}_{\mathrm{IW}}^{1}(K, D)\right) .
\end{array}
$$

In particular, Theorem 3.14 is equivalent to Theorem 3.21. 
Proof. Since we have $\mathbf{N}_{\text {rig }}(D)=\mathbf{B}_{\text {rig, }, K}^{\dagger} \otimes_{K} \mathbf{D}_{\text {crys }}^{K_{n}}(D)$ by Lemma 3.16, the principal fractional ideal

$$
\operatorname{det}_{\Lambda_{\infty}}\left(\mathbf{N}_{\mathrm{rig}}(D) \stackrel{\psi=1}{\operatorname{Exp}_{D, h}} \longrightarrow \mathbf{H}_{\mathrm{Iw}}^{1}(K, D)\right) \cdot \operatorname{char}_{\Lambda_{\infty}}\left(\mathbf{H}_{\mathrm{Iw}}^{2}\left(K, \mathbf{N}_{\text {rig }}(D)\right)\right)
$$

is equal to the product

$$
\begin{gathered}
\operatorname{det}_{\Lambda_{\infty}}\left(\left(\mathbf{B}_{\text {rig, } K}^{+} \otimes_{K} \mathbf{D}_{\text {crys }}^{K_{n}}(D)\right)^{\psi=1} \stackrel{\left.\operatorname{Exp}_{D, h}\right|_{\left(\mathbf{B}_{\text {rig }, K}^{+} \otimes_{K} \mathbf{D}_{\text {crys }}^{K_{n}}(D)\right)^{\psi=1}} ^{\longrightarrow}}{\longrightarrow} \mathbf{H}_{\mathrm{Iw}}^{1}(K, D)\right) . \\
\operatorname{char}_{\Lambda_{\infty}}\left(\left(\mathbf{B}_{\text {rig }, K}^{\dagger} \otimes_{K} \mathbf{D}_{\text {crys }}^{K_{n}}(D)\right)^{\psi=1} /\left(\mathbf{B}_{\text {rig }, K}^{+} \otimes_{K} \mathbf{D}_{\text {crys }}^{K_{n}}(D)\right)^{\psi=1}\right)^{-1} \cdot \operatorname{char}_{\Lambda_{\infty}}\left(\mathbf{H}_{\mathrm{IW}}^{2}\left(K, \mathbf{N}_{\text {rig }}(D)\right)\right) .
\end{gathered}
$$

Since we have

$$
\left(\mathbf{B}_{\text {rig }, K}^{+} \otimes_{K} \mathbf{D}_{\text {crys }}^{K_{n}}(D)\right) /(\psi-1)\left(\mathbf{B}_{\text {rig }, K}^{+} \otimes_{K} \mathbf{D}_{\text {crys }}^{K_{n}}(D)\right)=0
$$

by (3) of Lemma 3.17, using the snake lemma, we obtain the following isomorphisms:

$$
\begin{aligned}
\left(\mathbf{B}_{\text {rig }}^{\dagger} \otimes_{K} \mathbf{D}_{\text {crys }}^{K_{n}}(D)\right)^{\psi=1} /\left(\mathbf{B}_{\text {rig }, K}^{+} \otimes_{K} \mathbf{D}_{\text {crys }}^{K_{n}}(D)\right)^{\psi=} & \stackrel{\sim}{\rightarrow}\left(\mathrm{LA}\left(\mathbb{Z}_{p}, \mathbb{Q}_{p}\right) \otimes_{\mathbb{Q}_{p}} \mathbf{D}_{\text {crys }}^{K_{n}}(D)\right)^{\psi=1} \\
& \stackrel{\sim}{\rightarrow} \bigoplus_{k=0}^{k_{0}}\left(x^{k} \otimes \mathbf{D}_{\text {crys }}^{K_{n}}(D)\right)^{\psi=1},
\end{aligned}
$$

where the last isomorphism is (4) of Lemma 3.17 for sufficiently large $k_{0} \gg 0$. We similarly obtain an isomorphism

$$
\mathbf{H}_{\mathrm{Iw}}^{2}\left(K, \mathbf{N}_{\text {rig }}(D)\right) \stackrel{\sim}{\rightarrow} \bigoplus_{k=0}^{k_{0}}\left(x^{k} \otimes \mathbf{D}_{\text {crys }}^{K_{n}}(D)\right) /(1-\psi)\left(x^{k} \otimes \mathbf{D}_{\text {crys }}^{K_{n}}(D)\right) .
$$

Hence, we obtain

$$
\begin{aligned}
& \operatorname{char}_{\Lambda_{\infty}}\left(\left(\mathbf{B}_{\mathrm{rig}}^{\dagger} \otimes_{K} \mathbf{D}_{\mathrm{crys}}^{K_{n}}(D)\right)^{\psi=1} /\left(\mathbf{B}_{\mathrm{rig}, K}^{+} \otimes_{K} \mathbf{D}_{\mathrm{crys}}^{K_{n}}(D)\right)^{\psi=1}\right)^{-1} \cdot \operatorname{char}_{\Lambda_{\infty}}\left(\mathbf{H}_{\mathrm{Iw}}^{2}\left(K, \mathbf{N}_{\mathrm{rig}}(D)\right)\right) \\
& =\operatorname{det}_{\Lambda_{\infty}}\left(\bigoplus_{k=0}^{k_{0}} x^{k} \otimes \mathbf{D}_{\mathrm{crys}}^{K_{n}}(D) \stackrel{\psi-1}{\longrightarrow} \bigoplus_{k=0}^{k_{0}} x^{k} \otimes \mathbf{D}_{\mathrm{crys}}^{K_{n}}(D)\right)=\Lambda_{\infty}
\end{aligned}
$$

where the last equality follows from (v) of Lemma 3.12. Hence, we obtain an equality:

$$
\begin{aligned}
& \operatorname{det}_{\Lambda_{\infty}}\left(\mathbf{N}_{\text {rig }}(D) \psi=1 \stackrel{\operatorname{Exp}_{D, h}}{\longrightarrow} \mathbf{H}_{\mathrm{Iw}}^{1}(K, D)\right) \cdot \operatorname{char}_{\Lambda_{\infty}}\left(\mathbf{H}_{\mathrm{Iw}}^{2}\left(K, \mathbf{N}_{\text {rig }}(D)\right)\right) \\
& =\operatorname{det}_{\Lambda_{\infty}}\left(\left(\mathbf{B}_{\text {rig }, K}^{+} \otimes_{K} \mathbf{D}_{\text {crys }}^{K_{n}}(D)\right)^{\psi=1} \stackrel{\left.\operatorname{Exp}_{D, h}\right|_{\left(\mathbf{B}_{\text {rig }, K}^{+} \otimes_{K} \mathbf{D}_{\text {crys }}^{K_{n}}(D)\right)^{\psi=1}} ^{\longrightarrow}}{\longrightarrow} \mathbf{H}_{\mathrm{Iw}}^{1}(K, D)\right) \text {. }
\end{aligned}
$$

Next, we calculate the right-hand side of the proposition.

First, by the definition of $\Omega_{D, h}$ and by the property of $\operatorname{det}_{\Lambda_{\infty}}(-)$, the fractional ideal

$$
\operatorname{det}_{\Lambda_{\infty}}\left(\Lambda_{\infty} \otimes_{\mathbb{Q}_{p}} \mathbf{D}_{\text {crys }}^{K_{n}}(D) \stackrel{\Omega_{D, h}}{\longrightarrow} \mathbf{H}_{\mathrm{Iw}}^{1}(K, D)\right)
$$

is equal to the product

$$
\begin{gathered}
\operatorname{det}_{\Lambda_{\infty}}\left(\left(\mathbf{B}_{\mathrm{rig}, K}^{+} \otimes_{K} \mathbf{D}_{\mathrm{crys}}^{K_{n}}(D)\right)^{\psi=1} \stackrel{\left.\operatorname{Exp}_{D, h}\right|_{\left(\mathbf{B}_{\mathrm{rig}, K}^{+} \otimes_{K} \mathbf{D}_{\mathrm{crys}}^{\left.K_{n}(D)\right)^{\psi=1}}\right.} ^{\longrightarrow}}{\longrightarrow} \mathbf{H}_{\mathrm{Iw}}^{1}(K, D)\right) . \\
\operatorname{det}_{\Lambda_{\infty}}\left(\left(\left(\mathbf{B}_{\mathrm{rig}, K}^{+} \otimes_{K} \mathbf{D}_{\mathrm{crys}}^{K_{n}}(D)\right)^{\psi=1} \stackrel{1-\varphi}{\longrightarrow} \Lambda_{\infty} \otimes_{\mathbb{Q}_{p}} \mathbf{D}_{\mathrm{crys}}^{K_{n}}(D)\right)\right)^{-1}
\end{gathered}
$$


By Lemma 3.18, we have

$$
\begin{aligned}
\operatorname{det}_{\Lambda_{\infty}}\left(\left(\mathbf{B}_{\mathrm{rig}, K}^{+} \otimes_{K} \mathbf{D}_{\mathrm{crys}}^{K_{n}}(D)\right)^{\psi=1} \stackrel{1-\varphi}{\longrightarrow} \Lambda_{\infty} \otimes_{\mathbb{Q}_{p}} \mathbf{D}_{\mathrm{crys}}^{K_{n}}(D)\right) \\
=\operatorname{det}_{\Lambda_{\infty}}\left(\bigoplus_{k=0}^{k_{0}} t^{k} \otimes \mathbf{D}_{\mathrm{crys}}^{K_{n}}(D) \stackrel{1-\varphi}{\longrightarrow} \bigoplus_{k=0}^{k_{0}} t^{k} \otimes \mathbf{D}_{\mathrm{crys}}^{K_{n}}(D)\right)=\Lambda_{\infty} .
\end{aligned}
$$

Hence, we also obtain an equality:

$$
\begin{aligned}
& \operatorname{det}_{\Lambda_{\infty}}\left(\Lambda_{\infty} \otimes_{\mathbb{Q}_{p}} \mathbf{D}_{\text {crys }}^{K_{n}}(D) \stackrel{\Omega_{D, h}}{\longrightarrow} \mathbf{H}_{\mathrm{Iw}}^{1}(K, D)\right) \\
& \quad=\operatorname{det}_{\Lambda_{\infty}}\left(\left(\mathbf{B}_{\mathrm{rig}, K}^{+} \otimes_{K} \mathbf{D}_{\mathrm{crys}}^{K_{n}}(D)\right)^{\psi=1} \stackrel{\left.\left.\operatorname{Exp}_{D, h}\right|_{\left(\mathbf{B}_{\mathrm{rig}, K}^{+} \otimes_{K} \mathbf{D}_{\mathrm{crys}}^{\left.K_{n}(D)\right)^{\psi=1}}\right.} ^{\longrightarrow} \mathbf{H}_{\mathrm{Iw}}^{1}(K, D)\right),}{\longrightarrow}\right.
\end{aligned}
$$

which proves the proposition.

\section{List of notation}

Here is a list of the main notation of the article, in the order of the section in which it appears.

$\S 1.1: \exp _{K, V}, \exp _{K, V^{\vee}(1)}^{*}$.

$\S 1.2: \Lambda, \mathbf{H}_{\mathrm{Iw}}^{q}(K, V), \Omega_{V, h}$.

Notation: $p, K, K_{0}, \bar{K}, \mathbb{C}_{p}, v_{p},|-|_{p}, G_{K},\left\{\zeta_{p^{n}}\right\}_{n \geqq 0}, K_{n}, K_{\infty}, \chi, \Gamma_{K}, e_{1}, e_{k},|G|$.

$\S 2.1: \widetilde{\mathbf{E}}^{+}, v_{\widetilde{\mathbf{E}}^{+}}, \widetilde{\mathbf{E}}, \varepsilon, \widetilde{p}, \widetilde{\mathbf{A}}^{+}, \widetilde{\mathbf{A}}, \theta, \mathbf{B}_{\mathrm{dR}}^{+}, t, \mathbf{B}_{\mathrm{dR}}, \widetilde{\mathbf{B}}_{\mathrm{rig}}^{\dagger}, \widetilde{\mathbf{A}}^{[r, s]}, \widetilde{\mathbf{B}}^{[r, s]}, \mathbf{B}_{\mathrm{max}}^{+}, \widetilde{\mathbf{B}}_{\mathrm{rig}}^{\dagger, r}, \widetilde{\mathbf{B}}_{\mathrm{rig}}^{\dagger}$, $r_{n}, \iota_{n}: \widetilde{\mathbf{B}}_{\mathrm{rig}}^{\dagger, r_{n}} \hookrightarrow \mathbf{B}_{\mathrm{dR}}^{+}, \mathbf{B}_{\max }, \mathbf{B}_{e}, T, \mathbf{B}_{\mathrm{rig}, F}^{\dagger, r}, \mathbf{B}_{\mathrm{rig}, F}^{\dagger}, e_{K}, K_{0}^{\prime}, r(K), \pi_{K}, \mathbf{B}_{\mathrm{rig}, K}^{\dagger, r}$, $\mathbf{B}_{\text {rig, } K}^{\dagger}, \psi, n(K), \iota_{n}: \mathbf{B}_{\text {rig, } K}^{\dagger, r_{n}} \hookrightarrow K_{n}[[t]], \frac{1}{p} \operatorname{Tr}_{K_{n+1} / K_{n}},\left.D\right|_{L}, D^{\vee}, D_{1} \otimes D_{2}, n(D), D^{(n)}$, $\mathbf{D}_{\text {dif }}^{+}(D), \mathbf{D}_{\text {dif }, n}(D), \mathbf{D}_{\text {dif }}^{+}(D), \mathbf{D}_{\text {dif }}(D), K_{\infty}[[t]], K_{\infty}((t)), \iota_{n}: D^{(n)} \hookrightarrow \mathbf{D}_{\text {dif }, n}(D)$.

$\S 2.2: \Delta_{K}, \gamma_{K}, M^{\Delta_{K}}, C_{\gamma_{K}}^{\bullet}(M), C_{\varphi, \gamma_{K}}^{\bullet}(M), \mathrm{H}^{q}(K, D), \mathrm{H}^{q}(K, D[1 / t]), \mathrm{H}^{q}\left(K, \mathbf{D}_{\mathrm{dif}}^{+}(D)\right)$, $\mathrm{H}^{q}\left(K, \mathbf{D}_{\mathrm{dif}}(D)\right), \cup,\langle\rangle,, \mathrm{ev}, f_{\mathrm{tr}}, f_{\mathrm{tr}}^{\prime}, \kappa, \operatorname{rec}_{\mathbb{Q}_{p}}, C_{\psi, \gamma_{K}}^{\bullet}(D), \mathbf{D}_{\mathrm{crys}}^{K}(D), \mathbf{D}_{\mathrm{dR}}^{K}(D)$, $\mathrm{Fil}^{i} \mathbf{D}_{\mathrm{dR}}(D)$.

$\S 2.3: \delta_{1, D}, \quad \delta_{2, D}, \quad \widetilde{C}_{\varphi, \gamma_{K}}^{\bullet}\left(D^{(n)}\right), \quad \widetilde{C}_{\varphi, \gamma_{K}}^{\bullet}\left(D^{(n)}[1 / t]\right), \quad \widetilde{C}_{\varphi, \gamma_{K}}^{\bullet}\left(\mathbf{D}_{\mathrm{dif}, n}^{+}(D)\right), \quad \widetilde{C}_{\varphi, \gamma_{K}}^{\bullet}\left(\mathbf{D}_{\mathrm{dif}, n}(D)\right)$, $\exp _{K, D}$

$\S 2.4: \cup_{\mathrm{dif}}, g_{D}, \log (\chi),\langle,\rangle_{\mathrm{dif}}, \exp _{K, D^{\vee}(1)}^{*},[-,-]_{\mathrm{dR}}$.

$\S 2.5: W, W_{e}, W_{\mathrm{dR}}^{+}, W_{\mathrm{dR}}, W(V), W_{e}(D), W_{\mathrm{dR}}(D), W_{\mathrm{dR}}^{+}(D), W(D), \widetilde{D}^{(n)}(W), \widetilde{D}(W)$, $D(W), C^{q}\left(G_{K}, M\right), \delta_{q}, C^{\bullet}\left(G_{K}, M\right), C^{\bullet}\left(G_{K}, W\right), \mathrm{H}^{1}(K, W), \delta_{1, W}, \delta_{2, W}, \mathbf{D}_{\mathrm{dR}}^{K}(W)$, $\exp _{K, W}$.

$\S 3.1: \Gamma_{K, \text { tor }}, \Gamma_{K, \text { free }}, \Lambda_{n}, \Lambda_{\infty}, \mathbf{B}_{\text {rig, } \mathbb{Q}_{p}}^{+}, \widetilde{\Lambda}_{n}, \widetilde{\Lambda}_{n}^{\iota}, D \widehat{\otimes}_{\mathbb{Q}_{p}} \widetilde{\Lambda}_{n}^{\iota}, \mathbf{H}_{\mathrm{Iw}}^{q}(K, D), \widehat{\Gamma}_{K, \text { tor }}, \eta, \alpha_{\eta}$, $M_{\text {tor }}, A(\delta), f_{\delta}, \operatorname{pr}_{L, D(k)}, \delta_{L}, \mathbb{Q}_{p}\left[\widetilde{\Gamma_{K} / \Gamma_{L}}\right]^{\iota}, f_{D, k}, f_{k}, C_{\psi}^{\bullet}(D), \iota_{D}, p_{\Delta_{K}}, \log _{0}(-)$.

$\S 3.2: \nabla_{0}, \widehat{\Omega}_{\mathbf{B}_{\text {rig }, K}^{\dagger} / K_{0}^{\prime}}, \mathbf{N}_{\text {rig }}^{(n)}(D), \mathbf{N}_{\text {rig }}(D), \partial, \widetilde{\partial}$.

$\S 3.3: \nabla_{i}, \operatorname{Exp}_{D, h}, T_{L}, m(L)$.

§3.4: $\operatorname{char}_{\Lambda_{\infty}}(M), \operatorname{det}_{\Lambda_{\infty}}(f), \operatorname{det}_{\Lambda_{\infty}}\left(\mathrm{H}^{\bullet}(f)\right)$.

$\S 3.5: \mathbf{B}_{K, \text { rig }}^{+}, \mathrm{LA}_{h}\left(\mathbb{Z}_{p}, \mathbb{Q}_{p}\right), \operatorname{LA}\left(\mathbb{Z}_{p}, \mathbb{Q}_{p}\right),|-|_{h}$, Col, Res, $x^{k}, \widetilde{\Delta}, \Omega_{D, h}$. 
Acknowledgements. The author would like to thank Kenichi Bannai for constant encouragement. He also would like to thank Gaëtan Chenevier and Jonathan Pottharst for discussing related topics on $(\varphi, \Gamma)$-modules over the Robba ring.

\section{References}

1. Y. AndRÉ, Filtrations de Hasse-Arf et monodromie p-adique, Invent. Math. 148 (2002), $285-317$.

2. D. Benois, On Iwasawa theory of crystalline representations, Duke Math. J. 104 (2000), 211-267.

3. L. Berger, Représentations p-adiques et équations différentielles, Invent. Math. 148 (2002), 219-284.

4. L. Berger, Bloch and Kato's exponential map: three explicit formulas. Doc. Math, (2003), 99-129 (electronic). Kazuya Kato's fiftieth birthday.

5. L. Berger, Construction de $(\varphi, \Gamma)$-modules: représentations $p$-adiques et $B$-paires, Algebra Number Theory 2(1) (2008), 91-120.

6. L. BERGER, Équations différentielles p-adiques et $(\varphi, N)$-modules filtres, Astérisque (319) (2008), 13-38, Représentations $p$-adiques de groupes $p$-adiques. I. Représentations galoisiennes et $(\varphi, N)$-modules.

7. S. BLOCH AND K. Kato, $L$-functions and Tamagawa numbers of motives, in The Grothendieck Festschrift, Vol. I, Progress in Mathematics, Volume 86, pp. 333-400 (Birkhäuser Boston, Boston, MA, 1990).

8. F. Cherbonnier And P. Colmez, Représentations p-adiques surconvergentes, Invent. Math. 133 (1998), 581-611.

9. F. Cherbonnier And P. Colmez, Théorie d'Iwasawa des représentations p-adiques dun corps local, J. Amer. Math. Soc. 12 (1999), 241-268.

10. G. Chenevier, Sur la densité des representations cristallines de $\operatorname{Gal}\left(\overline{\mathbb{Q}}_{p} / \mathbb{Q}_{p}\right)$, Math Ann. (2012).

11. P. Colmez, Théorie d'Iwasawa des représentations de de Rham d'un corps local, Ann. of Math. 148 (1998), 485-571.

12. R. CREw, Finiteness theorems for the cohomology of an overconvergent isocrystal on a curve, Ann. Sci. Éc. Norm. Supér. (4) 31(6) (1998), 717-763.

13. J.-M. Fontaine, Représentations p-adiques des corps locaux I, in The Grothendieck Festschrift, Vol. 2, Progress in Mathematics, Volume 87, pp. 249-309 (Birkhäuser, Boston, 1990).

14. J.-M. Fontaine, Le corps des périodes p-adiques, Astérisque 223 (1994), 59-111.

15. J.-M. Fontaine, Presque $\mathbb{C}_{p}$-représentations, Kazuya Kato's fifties birthday, Doc. Math., (2003), Extra Vol., 285-385 (electronic).

16. L. Herr, Sur la cohomologie galoisienne des corps p-adiques, Bull. Soc. Math. France 126(4) (1998), 563-600.

17. L. Herr, Une approche nouvelle de la dualité locale de Tate, Math. Ann. 320(2) (2001), 307-337.

18. K. Kato, Lectures on the approach to Iwasawa theory for Hasse-Weil $L$-functions via $\mathbf{B}_{\mathrm{dR}}$, in Arithmetic algebraic geometry, Lecture Notes in Mathematics, Volume 1553, pp. 50-63 (Springer, Berlin, 1993).

19. K. Kato, Lectures on the approach to Iwasawa theory for Hasse-Weil $L$-functions via $\mathbf{B}_{\mathrm{dR}}$. II, preprint (1993).

20. K. KATO, p-adic Hodge theory and values of zeta functions of modular forms, Astérisque ix(295) (2004), 117-290, Cohomologies p-adiques et applications arithmétiques. III. 
21. K. Kato, M. Kurihara and T. Tsuji, Local Iwasawa theory of Perrin-Riou and syntomic complexes, preprint (1996).

22. K. Kedlaya, A p-adic local monodromy theorem, Ann. of Math. (2) 160 (2004), 93-184.

23. R. Liu, Cohomology and duality for $(\varphi, \Gamma)$-modules over the Robba ring, Int. Math. Res. Not. IMRN (3) (2008).

24. Z. Mebkhout, Analogue $p$-adique du théorème de Turrittin et le théorème de la monodromie p-adique, Invent. Math. 148 (2002), 319-351.

25. K. Nakamura, Classification of two dimensional split trianguline representations of p-adic fields, Compositio Math. 145 (2009), 865-914.

26. K. Nakamura, Deformations of trianguline $B$-pairs and Zariski density of two dimensional crystalline representations, preprint (arXiv:1006.4891 [math.NT]).

27. K. Nakamura, A generalization of Kato's local $\varepsilon$-conjecture for $(\varphi, \Gamma)$-modules over the Robba ring, in preparation.

28. B. Perrin-Riou, Théorie d'Iwasawa et hauteurs p-adiques, Invent. Math. 109 (1992), $137-185$.

29. B. Perrin-Riou, Théorie d'Iwasawa des représentations p-adiques sur un corps local, Invent. Math. 115 (1994), 81-161.

30. B. PerRin-Riou, Fonctions $L p$-adiques des représentations $p$-adiques, Astérisque (229)(1995), 198 pp.

31. J. Pottharst, Analytic families of finite-slope Selmer groups, preprint on his web page.

32. J. Pottharst, Cyclotomic Iwasawa theory of motives, preprint on his web page.

33. P. Schneider and J. Teitelbaum, Algebras of $p$-adic distributions and admissible representations, Invent. Math. 153(1) (2003), 145-196. 